\title{
O-minimal de Rham cohomology
}

by

Rodrigo Figueiredo

A thesis submitted in partial fulfillment for the degree of Doctor of Philosophy in Mathematics

in the

Instituto de Matemática e Estatística da

Universidade de São Paulo

December 2017 



\section{Declaration of Authorship}

I, Rodrigo Figueiredo, declare that this thesis entitled "O-minimal de Rham cohomology" and the work presented in it are my own. I confirm that:

- This work was done wholly or mainly while in candidature for a research degree at this University.

- Where any part of this thesis has previously been submitted for a degree or any other qualification at this University or any other institution, this has been clearly stated.

- Where I have consulted the published work of others, this is always clearly attributed.

- Where I have quoted from the work of others, the source is always given. With the exception of such quotations, this thesis is entirely my own work.

- I have acknowledged all main sources of help.

- Where the thesis is based on work done by myself jointly with others, I have made clear exactly what was done by others and what I have contributed myself. 



\section{Abstract}

The aim of this dissertation lies in establishing an o-minimal de Rham cohomology theory for abstract-definable $\mathcal{C}^{\infty}$ manifolds in an o-minimal expansion of the real field which admits smooth cell decomposition and defines the exponential function, by following the classical de Rham cohomology. We can specify the o-minimal cohomology groups and attain some properties as the existence of Mayer-Vietoris sequence and the invariance under abstract-definable $\mathcal{C}^{\infty}$ diffeomorphisms. However, in order to obtain the invariance of our o-minimal cohomology under abstractdefinable homotopy we must, working in a tame context that defines sufficiently many primitives, assume the validity of a statement related to Bröcker's problem. 


\section{Acknowledgements}

First and foremost, I would like to express my indebtedness and render my warmest thanks to my advisor, Professor Ricardo Bianconi, who made this work possible. His friendly guidance and expert advice have been invaluable during my time as a PhD student at IME-USP.

I extend my thankfulness to the other members of my thesis committee - Professor Hugo Luiz Mariano, Professor Vinicius Cifú Lopes, Professor Francisco Miraglia Neto and Professor Andreas Bernhard Michael Brunner - for their insightful comments on this dissertation.

I gratefully acknowledge CNPq for providing financial support throughout the elaboration of this thesis.

My time at USP was made enjoyable in large part due to the many friends I made there and that became a part of my life. I am grateful for each second spent with all of them, including my roommates.

Lastly, a deep gratitude goes to my family - my parents Elza and Isaias, my brother Robson and my sister Jackie - who have been supporting me in all my pursuits. Special thanks also go to my loving, encouraging and patient fiancée Carla, whose continuous support particularly in the final stages of my $\mathrm{PhD}$ program will be forever appreciated.

Rodrigo Figueiredo

December 15th, 2017 


\section{Contents}

Declaration of Authorship iii

$\begin{array}{ll}\text { Abstract } & \text { V }\end{array}$

Acknowledgements vi vi vis

1 Introduction 1

2 Abstract-definable manifolds $\quad 7$

2.1 Abstract-definable manifolds . . . . . . . . . . . . . . . . 7

2.2 Some topological notions and facts . . . . . . . . . . . . 17

3 Abstract-definable partitions of unity 27

3.1 Abstract-definable partitions of unity . . . . . . . . . . . . . 28

3.2 Abstract-definable bump functions and other consequences . . . . . 32

4 The tangent and cotangent bundles $\quad 37$

4.1 The tangent bundle . . . . . . . . . . . . . . . . . 37

4.2 The cotangent bundle . . . . . . . . . . . . . . . . . 44

5 Abstract-definable vector bundles $\quad 47$

5.1 Abstract-definable vector bundles . . . . . . . . . . . . . . . . 47

5.2 Sections and frames . . . . . . . . . . . . . . . . . . 49

6 Abstract-definable forms $\quad 55$

6.1 Abstract-definable 1-forms . . . . . . . . . . . . 56

6.2 Abstract-definable $k$-forms . . . . . . . . . . . . . . . 62

$\begin{array}{lll}7 & \text { Exterior derivative } & \mathbf{7 1}\end{array}$

7.1 Local operators . . . . . . . . . . . . . . . . . . . 73

7.2 Existence and uniqueness of a local exterior derivative . . . . . . . . 74

7.3 Existence and uniqueness of a global exterior derivative . . . . . . . 77 
8 O-minimal de Rham cohomology $\quad 83$

8.1 O-minimal de Rham cohomology . . . . . . . . . . . . . . . . . 84

8.2 Abstract-definable homotopy invariance . . . . . . . . . . . . . . 90

$\begin{array}{lr}\text { A Multilinear algebra } & 101\end{array}$

A.1 Wedge product . . . . . . . . . . . . . . . . . . 101

A.2 Graded algebra . . . . . . . . . . . . . . . 103

$\begin{array}{lr}\text { B Homological algebra } & 105\end{array}$

B.1 Cochain complexes . . . . . . . . . . . . . . . . . . 105

B.2 Cochain homotopy . . . . . . . . . . . . . . . . 108

$\begin{array}{lr}\text { Bibliography } & 109\end{array}$

$\begin{array}{lr}\text { Index } & 112\end{array}$ 
To my parents 



\section{Chapter 1}

\section{Introduction}

O-minimal structures have their roots in the early 80's in the work [4]. In that paper, L. van den Dries, before discussing the question raised by Tarski in his monograph [28] of whether the elementary theory of the exponential field $\langle\mathbb{R},+, \cdot, \exp \rangle$ is decidable, derives some finiteness properties of sets definable in an expansion of $\langle\mathbb{R},<\rangle$ of finite type (i.e., one within the definable subsets of $\mathbb{R}$ are unions of a finite set and finitely many intervals). Soon afterwards, in a series of three papers [24], [14], and [25], A. Pillay, C. Steinhorn and J. Knight give a systematized treatment of expansions of a dense linear order without endpoints that have the strong condition of "every definable set with parameters is a finite union of intervals and points", under the coinage of o-minimal structures, extending the work of L. van den Dries, among other things. Since then, the interest of logicians and mathematicians for this area has been increasing immensely, as well as its relation with other branches of mathematics.

O-minimality found deep connections with diophantine geometry in the first decade of 21st century, beginning with the study carried out by J. Pila and A. Wilkie of rational points in a definable set [23] and culminating in the unconditional proof of André-Oort conjecture for arbitrary products of modular curves [21] by J. Pila. Postliminary works, for instance [22] and [12], presenting solutions for special cases of this conjecture have also used o-minimality in a crucial way; also, in a recent paper [32] A. Wilkie raises diophantine questions in the spirit of those addressed in [23], which somehow shows that applications of this fragment of model theory to algebraic geometry are far from having been exhausted. 
Linked up with algebraic geometry although in a different direction, M. Edmundo developed a cohomology theory for the category of definable manifolds and continuous maps within the framework of an o-minimal expansion of a real closed field [7], and used this to solve a problem proposed by Y. Peterzil and C. Steinhorn [20], concerning the existence of torsion points on definably compact definable abelian groups. (Here "definable manifold" is, in our parlance, an abstractdefinable $\mathcal{C}^{0}$ manifold - see Definition 2.6.) Subsequently, M. Edmundo, G. Jones and N. Peatfield established a sheaf cohomology for the category of definable sets in an o-minimal expansion of a group [9]; and, working in the category where the sets and continuous maps are all definable in an arbitrary o-minimal structure with definable Skolem functions, M. Edmundo and N. Peatfield constructed a Cech cohomology theory [8]. In view of all these results settling cohomologies for (abstract-)definable objects, we inquire about the existence of a definable analogue of the de Rham cohomology on a tame category.

In the present dissertation we elaborate a de Rham-like cohomology theory for abstract-definable $\mathcal{C}^{\infty}$ manifolds in the setting of an o-minimal expansion of the real field which, in addition to possessing smooth cell decomposition, defines the exponential function, and show that such a cohomology only has the strong property of homotopy invariance if we restrict ourselves to an o-minimal environment that defines sufficiently many functions. Our program is to follow the lines of the construction of the classical de Rham cohomology starting from the general context of abstract-definable $\mathcal{C}^{p}$ manifolds $(p<\infty)$ where the fixed framework is an arbitrary o-minimal expansion of a real closed field, and push it to the limit. These abstract-definable $\mathcal{C}^{p}$ manifolds generalize the notion of abstract $\mathcal{C}^{p}$ Nash manifolds [26], since the transition maps might have additional parts other than the semi-algebraic.

We refer the reader to [5] and [6] for an introduction to o-minimal structures.

This work is organized as most of the expository texts on smooth manifolds.

We fix an o-minimal expansion of a real closed field and introduce in Chapter 2 the notion of an abstract-definable manifold $M$ of class $\mathcal{C}^{p}$, with $p<\infty$, and prove some basic topological facts concerning the manifold topology, some of them quite similar to the classical case such as, for example, every abstract-definable $\mathcal{C}^{p}$ manifold is definably regular, locally definably compact; and some others different 
such as every abstract-definable $\mathcal{C}^{p}$ manifold has finitely many definably connected components.

Chapter 3 is where the main technical difficulty of this work lies in, and it is devoted to the construction of an abstract-definable version of partitions of unity (subordinate to an abstract-definable $\mathcal{C}^{p}$ atlas) and some of their consequences, as the existence of abstract-definable $\mathcal{C}^{p}$ bump functions. Unlike in the classical setting in which a partition of unity subordinate to a fixed open cover of a smooth manifold is built upon the employment of tools such as smooth bump functions and the existence of a countable basis for a smooth manifold - both of them unavailable for us at this point -, we adapt a method by A. Fischer [11], used to attain partitions of unity within the framework of an o-minimal expansion of the real field that admits smooth cell decomposition and in addition defines the exponential function, that makes heavy use of the finiteness of the atlas, and a weaker form of the definable $\mathcal{C}^{p}$ Urysohn's lemma having a definable open set $U \subseteq R^{m}$ as the background topological space (Lemma 3.3). In order to obtain this weak Urysohn's lemma, A. Fisher settles a result concerning the approximation of definable continuous functions by definable $\mathcal{C}^{p}$ functions, where $0<p \leq \infty$. A. Thamrongthanyalak proves, in a paper published in 2013 ([29]), an analogous approximation theorem for o-minimal expansions of a real closed field, and for $0<p<\infty$, enabling the technique in question to be applied within our context. (Observe that if the concerned definable open set $U$ were the whole $R^{m}$, we would be done by Corollary C.12 [6], with no need of any approximation result at all.)

After providing the foundations, we proceed to establish in Chapter 4 the tangent space $T_{x} M$ of an abstract-definable $\mathcal{C}^{p}$ manifold $M$ at a point $x$ in $M$ by following [19], and its corresponding cotangent space $T_{x}^{*} M$.

In Chapter 5, we introduce the notion of an abstract-definable $\mathcal{C}^{p}$ vector bundle, which is entirely analogous to the classical case, except that the number of the local trivializations is finite and the maps involved are abstract-definable $\mathcal{C}^{p}$. In a similar fashion, we bring in the concept of abstract-definable $\mathcal{C}^{p}$ sections, and give a local description of some of them.

In Chapter 6 we present the core element in the study of the o-minimal de Rham cohomology theory, the abstract-definable $\mathcal{C}^{p} k$-forms with $k \geq 0$, and give a characterization of these special abstract-definable $\mathcal{C}^{p}$ sections in terms of the coordinate frames. This is used to prove, among other things, that the pullback of 
abstract-definable $\mathcal{C}^{p} k$-forms under an abstract-definable $\mathcal{C}^{p}$ map remain abstractdefinable $k$-forms, despite being of class $\mathcal{C}^{p-1}$.

In Chapter 7, by reason of the impossibility presented in the 6th chapter, we turn our attention to smooth abstract-definable forms, which requires that we work in an o-minimal expansion $\mathcal{R}$ of the real field which admits cell decomposition, and also defines the exponential function, provided that we want to exploit what we produced so far. Following the classical case, we verify the existence and uniqueness of an exterior derivative on the vector space of all abstract-definable $\mathcal{C}^{\infty}$ forms. This exterior derivative is tame, in the sense that the abstract-definability of the forms is preserved. Moreover it commutes with the pullback of an abstractdefinable $\mathcal{C}^{\infty}$ map.

Finally, in Chapter 8, we specify the $k$ th o-minimal de Rham cohomology groups, and demonstrate that the o-minimal de Rham cohomology satisfies all analogous main theorems for classical de Rham cohomology except the Hotomopy Axiom (Theorem 8.14). Specifically, such a statement does not hold within the exponential real field $\mathbb{R}_{\text {exp }}$. We finish this chapter by showing that the Homotopy Axiom is fulfilled, therefore so is the Poincare Lemma (Corollary 8.17), whenever the fixed setting is the Pfaffian closure of $\mathcal{R}$.

We follow very closely [30] in the development of the Chapters 5-8, although picking only the contents that were enough to establish our cohomology.

From Chapter 2 until Chapter 6, $\mathcal{R}$ denotes an o-minimal expansion of an arbitrary real closed field $\langle R,>, 0,1,+,-, \cdot\rangle$. By "definable" we mean "definable in $\mathcal{R}$ with parameters in $R$ ", unless otherwise stated. Moreover, $p$ denotes a nonnegative integer, hence $p<\infty$. 


\section{Notation.}

- $\mathbb{N}$ denotes the set of nonnegative integers, $\mathbb{R}$ the field of real numbers, and $\emptyset$ the empty set.

- For any set $X, i d_{X}$ denotes the identity map $x \mapsto x$ on $X$.

- The $m$-tuple $\left(r^{1}, \ldots, r^{m}\right)$ indicates the coordinates of a point in $R^{m}$ in the standard basis.

- Given a topological space $X$ and a subset $Y$ of $X$, by $\operatorname{int}_{X}(Y), c l_{X}(Y)$, and $b d_{X}(Y)$ we mean the topological interior, closure and boundary of $Y$ in $X$ respectively; when the topological space $X$ is clear from the context, we drop the letter $X$ in these symbols.

- For any map $f: A \rightarrow B, \Gamma(f)$ denotes its graph $\{(x, f(x)): x \in A\}$.

- Given a map $f$ from an interval $I$ to a topological space $X$ and a limit point a of $I$, we denote by $\lim _{t \rightarrow a^{+}} f(t)$ and $\lim _{t \rightarrow a^{-}} f(t)$ the right- and left-handed limits of $f$ at a, respectively.

- The collection of all functions from a set $X$ to $R$ will be denoted by $\mathcal{F}(X)$. $\mathcal{F}(X)$ is made into a commutative ring with identity when endowed with pointwise sum and multiplication.

- The expression " $A \cong B$ " indicates the existence of an isomorphism between the objects $A$ and $B$ of a certain category. For instance, if we are dealing with vector spaces, then " $A \cong B$ " means that "there is a vector space isomorphism between the vector spaces $A$ and $B$ ".

Further notations are explained along the text. 



\section{Chapter 2}

\section{Abstract-definable manifolds}

In this chapter we introduce the notions of abstract-definable $\mathcal{C}^{p}$ manifold and abstract-definable sets and maps, by following the work of A. Berarducci and M. Otero [1], and prove some simple facts concerning these concepts. In the second part, we describe some first-order topological notions and properties, analogous to the classical ones, inherited by abstract-definable manifolds. Some of them are used in this and further chapters, as the definable normality of the abstractdefinable $\mathcal{C}^{p}$ manifolds and the fact that these have finitely many definably connected components.

\subsection{Abstract-definable manifolds}

Recall from Section 10 ([1], p. 114) the following.

Definition 2.1. Let $M$ be a set, and let $\left\{\phi_{i}: U_{i} \rightarrow \phi_{i}\left(U_{i}\right) \subseteq R^{m}\right\}_{i \in I}$ be a finite nonempty family of set-theoretic bijections where each $U_{i}$ is a subset of $M$ and $\phi_{i}\left(U_{i}\right)$ is a definable open set in $R^{m}$, called charts on $M$. We say that such a collection is an abstract-definable $\mathcal{C}^{p}$ atlas on $M$ of dimension $m$, with $0 \leq$ $p<\infty$, if $M=\bigcup_{i \in I} U_{i}$ and any two charts $\phi_{i}, \phi_{j}$ are $\mathcal{C}^{p}$-compatible, that is, $\phi_{i}\left(U_{i} \cap U_{j}\right)$ and $\phi_{j}\left(U_{i} \cap U_{j}\right)$ are definable and open in $R^{m}$, and the transition $\operatorname{map} \phi_{j} \circ \phi_{i}^{-1}: \phi_{i}\left(U_{i} \cap U_{j}\right) \rightarrow \phi_{j}\left(U_{i} \cap U_{j}\right)$ is a definable $\mathcal{C}^{p}$-diffeomorphism. (Here, by a definable $\mathcal{C}^{0}$-diffeomorphism we mean a definable homeomorphism.) 
Remark 2.2. We adopt the convention that the empty map is a definable $\mathcal{C}^{p}$ diffeomorphism, and as a consequence $\phi_{i}$ and $\phi_{j}$ in the above definition are always $\mathcal{C}^{p}$-compatible in the case $U_{i} \cap U_{j}=\emptyset$.

In order to show that two charts $\phi_{i}$ and $\phi_{j}$ are $\mathcal{C}^{p}$-compatible, it is enough to check the transitions maps $\phi_{j} \circ \phi_{i}^{-1}$ and $\phi_{i} \circ \phi_{j}^{-1}$ are definable $\mathcal{C}^{p}$, since one is the inverse of the other.

Notation. A chart $\phi: U \rightarrow \phi(U) \subseteq R^{m}$ will often be denoted by $(U, \phi)$. Another recurrent notation for such a chart is $\left(U, x^{1}, \ldots, x^{m}\right)$, where $x^{i}:=p r_{i} \circ \phi$ and $\operatorname{pr}_{i}: R^{m} \rightarrow R$ indicates the projection onto the ith coordinate.

Along the whole text, we will use the terminology: given a set $M$ and a point $x \in M$, by "a chart on $M$ at $x$ " we mean "a chart on $M$ whose domain contains $x "$

Lemma 2.3. Let $M$ be a set, $\mathcal{A}:=\left\{\phi_{i}: U_{i} \rightarrow \phi_{i}\left(U_{i}\right) \subseteq R^{m}\right\}_{i \in I}$ an abstractdefinable $\mathcal{C}^{p}$ atlas on $M$, and $\psi: V \rightarrow \psi(V) \subseteq R^{m}, \varphi: W \rightarrow \varphi(W) \subseteq R^{m}$ charts on $M$. Suppose $\psi$ and $\varphi$ are both $\mathcal{C}^{p}$-compatible with $\mathcal{A}$, i.e., $\mathcal{C}^{p}$-compatible with every chart in $\mathcal{A}$. Then $\psi$ is $\mathcal{C}^{p}$-compatible with $\varphi$.

Proof. For each $i, \psi$ is $\mathcal{C}^{p}$-compatible with $\phi_{i}$, which in turn is $\mathcal{C}^{p}$-compatible with $\varphi$. We claim that $\psi\left(V \cap U_{i} \cap W\right), \varphi\left(V \cap U_{i} \cap W\right)$ are open in $R^{m}$ and $\varphi \circ \psi^{-1}$ is a definable $\mathcal{C}^{p}$ map on $\psi\left(V \cap U_{i} \cap W\right)$. The first part of the claim follows immediately from the identities

$$
\psi\left(V \cap U_{i} \cap W\right)=\left(\psi \circ \phi_{i}^{-1}\right)\left(\phi_{i}\left(V \cap U_{i}\right) \cap \phi_{i}\left(U_{i} \cap W\right)\right)
$$

and

$$
\varphi\left(V \cap U_{i} \cap W\right)=\left(\varphi \circ \phi_{i}^{-1}\right)\left(\phi_{i}\left(V \cap U_{i}\right) \cap \phi_{i}\left(U_{i} \cap W\right)\right) .
$$

As for the second part, note that $\varphi \circ \psi^{-1}: \psi\left(V \cap U_{i} \cap W\right) \rightarrow \varphi\left(V \cap U_{i} \cap W\right)$ is the following composition of definable $\mathcal{C}^{p}$ maps

$$
\left.\left(\varphi \circ \phi_{i}^{-1}\right) \circ\left(\phi_{i} \circ \psi^{-1}\right)\right|_{\psi\left(V \cap U_{i} \cap W\right)},
$$

hence it is also a definable $\mathcal{C}^{p}$ map. Now, since $\psi(V \cap W)$ is covered by the finitely many open sets $\psi\left(V \cap U_{i} \cap W\right)$ in $R^{m}$ with $i \in I$, and $\varphi \circ \psi^{-1}$ restricted to each such a set is definable $\mathcal{C}^{p}$, it follows that $\varphi \circ \psi^{-1}: \psi(V \cap W) \rightarrow \varphi(V \cap W)$ is definable $\mathcal{C}^{p}$. 
Proposition 2.4. Suppose $M$ is a set and $\mathcal{A}, \mathcal{B}$ are two abstract-definable $\mathcal{C}^{p}$ atlases on $M$. The following holds.

(1) $\mathcal{A} \cup \mathcal{B}$ is an abstract-definable $\mathcal{C}^{p}$ atlas if and only if each $\phi \in \mathcal{A}$ is $\mathcal{C}^{p}$ compatible with every $\psi \in \mathcal{B}$.

(2) The relation $\sim$ defined on the set of all abstract-definable $\mathcal{C}^{p}$ atlases on $M$ by

$$
\mathcal{A} \sim \mathcal{B} \stackrel{\text { def }}{\Leftrightarrow} \mathcal{A} \cup \mathcal{B} \text { is an abstract-definable } \mathcal{C}^{p} \text { atlas on } M
$$

is an equivalence relation.

Proof. The assertion (1) follows immediately from the definitions of abstractdefinable $\mathcal{C}^{p}$ atlas and $\mathcal{C}^{p}$-compatibility. To achieve (2), we will only check the transitivity of $\sim$, since reflexivity and symmetry are straightforward. Let $\mathcal{A} \sim \mathcal{B}$ and $\mathcal{B} \sim \mathcal{D}$ be abstract-definable $\mathcal{C}^{p}$ atlases on $M$, and consider arbitrary charts $\phi \in \mathcal{A}, \psi \in \mathcal{D}$. Since both $\phi$ and $\psi$ are $\mathcal{C}^{p}$-compatible with $\mathcal{B}$, they are $\mathcal{C}^{p}$ compatible with one each other, by Lemma 2.3. Therefore $\mathcal{A} \cup \mathcal{D}$ is an abstractdefinable $\mathcal{C}^{p}$ atlas.

Notation. Throughout the text the symbol $\sim$ will denote the equivalence relation in (2.1), unless otherwise stated.

For a given set $M$ and an abstract-definable $\mathcal{C}^{p}$ atlas $\mathcal{A}:=\left\{\phi_{i}: U_{i} \rightarrow \phi_{i}\left(U_{i}\right) \subseteq\right.$ $\left.R^{m}\right\}_{i \in I}$ on $M$, there is a unique topology $\tau_{\mathcal{A}}$ in which each $U_{i}$ is open and every $\phi_{i}$ is a homeomorphism. Namely,

$$
U \in \tau_{\mathcal{A}} \stackrel{\text { def }}{\Leftrightarrow} \phi_{i}\left(U_{i} \cap U\right) \text { is open in } R^{m} \text {, for all } i \in I .
$$

In the sequel, we show that two $\sim$-equivalent abstract-definable $\mathcal{C}^{p}$ atlases on $M$ induce the same topology given by $(2.2)$.

Lemma 2.5. Let $M$ be a set, and let $\mathcal{A}, \mathcal{B}$ be two $\sim$-equivalent abstract-definable $\mathcal{C}^{p}$ atlases on $M$. Then the induced topologies $\tau_{\mathcal{A}}$ and $\tau_{\mathcal{B}}$ over $M$, given by (2.2), agree with each other.

Proof. Denote by $\tau$ the topology induced by $\mathcal{A} \cup \mathcal{B}$. By definition, for each chart $(U, \phi) \in \mathcal{A}$ the set $U$ is open and the map $\phi$ is a homeomorphism with respect to 
both topologies $\tau_{\mathcal{A}}$ and $\tau$. Similarly, for each $(V, \psi) \in \mathcal{B}$ the set $V$ is open and $\psi$ is a homeomorphism in $\tau_{\mathcal{B}}$ and $\tau$. By the definition of $\tau_{\mathcal{A}}$ and $\tau_{\mathcal{B}}$, we thus get $\tau_{\mathcal{A}}=\tau=\tau_{\mathcal{B}}$

In view of Lemma 2.5 every atlas lying in a fixed $\sim$-equivalence class of abstractdefinable $\mathcal{C}^{p}$ atlases on $M$ induces the same topology. Thus, we call the manifold topology over $M$, the topology induced, as in (2.2), by any representative of a fixed $\sim$-equivalence class of abstract-definable $\mathcal{C}^{p}$ atlases on $M$.

Definition 2.6. An abstract-definable $\mathcal{C}^{p}$ manifold of dimension $m$ is a set $M$ together with a $\sim$-equivalence class of $m$-dimensional abstract-definable $\mathcal{C}^{p}$ atlases on $M$, whose manifold topology is Hausdorff.

We denote an abstract-definable $\mathcal{C}^{p}$ manifold by simply a pair $(M, \mathcal{A})$, where $M$ is its underlying set and $\mathcal{A}$ is a representative of the given $\sim$-equivalence class. When $\mathcal{A}$ is clear from the context, the abstract-definable $\mathcal{C}^{p}$ manifold is designated just by its underlying set. Note that in order to show that a set $M$ can be made into an abstract-definable $\mathcal{C}^{p}$ manifold it suffices to exhibit an abstract-definable $\mathcal{C}^{p}$ atlas on $M$, and check that the corresponding manifold topology is Hausdorff.

Example 2.1. For each $n \in \mathbb{N}$ the set $R^{n}$ with the atlas $\left\{\left(R^{n}, \operatorname{id}_{R^{n}}\right)\right\}$, where $\operatorname{id}_{R^{n}}$ is the identity map on $R^{n}$, is an abstract-definable $\mathcal{C}^{p}$ manifold.

Example 2.2 (Abstract-definable manifold of dimension 0). Recall that $R^{0}:=$ $\{0\}$. If $M$ is a finite set, say $\left\{x_{1}, \ldots, x_{n}\right\}$, then $\left\{x_{i} \mapsto 0\right\}_{i=1}^{n}$ is an abstract-definable $\mathcal{C}^{p}$ atlas on $M$. The manifold topology on $M$ is the discrete topology, hence Hausdorff. Conversely, if $\left(M,\left\{\phi_{i}: U_{i} \rightarrow \phi_{i}\left(U_{i}\right) \subseteq R^{0}\right\}_{i \in I}\right)$ is a 0 -dimensional abstractdefinable $\mathcal{C}^{p}$ manifold, then $I$ is finite and each $U_{i}$ is a singleton. Therefore, $M$ is finite.

Example 2.3 (Projective space). Let $m \in \mathbb{N}$, and denote by $\mathbb{P}^{m}(R)$ the set of 1-dimensional vector subspaces of $R^{m+1}$ (i.e., the set of all lines in $R^{m+1}$ passing through the origin). For each $i \in\{0, \ldots, m\}$, denote by $U_{i}$ the set

$$
\left\{x:=\left(x_{0}: \cdots: x_{m}\right) \in \mathbb{P}^{m}(R): x_{i} \neq 0\right\} .
$$

Then, the maps $\phi_{i}: U_{i} \rightarrow R^{m}$ given by

$$
\phi_{i}(x):=\left(\frac{x_{0}}{x_{i}}, \cdots, \frac{\widehat{x_{i}}}{x_{i}}, \cdots, \frac{x_{m}}{x_{i}}\right) \text { with } i=0, \ldots, m
$$


form an abstract-definable $\mathcal{C}^{p}$ atlas on $\mathbb{P}^{m}(R)$. Here, $\widehat{x_{i} / x_{i}}$ means the quotient $x_{i} / x_{i}$ is omitted.

Example 2.4 (Sphere). Let $m \in \mathbb{N}$ and let $S^{m}$ be the set

$$
\left\{x \in R^{m+1}:|x|=1\right\} .
$$

Denote by $U_{i}^{+}$the set $\left\{x \in S^{m}: x_{i}>0\right\}$ and by $U_{i}^{-}$the set $\left\{x \in S^{m}: x_{i}<0\right\}$, for each $i=1, \ldots, m+1$. Also, let $h_{i}^{ \pm}: U_{i}^{ \pm} \rightarrow\left\{x \in R^{m}:|x|<1\right\}$ be the projection

$$
\left(x_{1}, \ldots, x_{m+1}\right) \mapsto\left(x_{1}, \ldots, \widehat{x}_{i}, \ldots, x_{m+1}\right),
$$

where $\widehat{x}_{i}$ designates the coordinate $x_{i}$ is omitted. The collection $\left\{h_{i}^{+}, h_{i}^{-}: i=\right.$ $1, \ldots, m\}$ is an abstract-definable $\mathcal{C}^{m}$ atlas on $S^{m}$.

Example 2.5 (Product of abstract-definable manifolds). Suppose $\left(M,\left\{\phi_{i}: U_{i} \rightarrow\right.\right.$ $\left.\left.\phi_{i}\left(U_{i}\right)\right\}_{i \in I}\right)$ and $\left(N,\left\{\psi_{j}: V_{j} \rightarrow \psi_{j}\left(V_{j}\right)\right\}_{j \in J}\right)$ are abstract-definable $\mathcal{C}^{p}$ manifolds of dimension $m$ and $n$, respectively. Then the pair $\left(M \times N,\left\{\phi_{i} \times \psi_{j}\right\}_{(i, j) \in I \times J}\right)$, with $\phi_{i} \times \psi_{j}: U_{i} \times V_{j} \rightarrow \phi_{i}\left(U_{i}\right) \times \psi_{j}\left(V_{j}\right)$ given by

$$
\left(\phi_{i} \times \psi_{j}\right)(x, y):=\left(\phi_{i}(x), \psi_{j}(y)\right)
$$

is an abstract-definable $\mathcal{C}^{p}$ manifold of dimension $m+n$.

Example 2.6 (Torus). Examples 2.4 and 2.5 imply that the $m$-torus

$$
T^{m}:=S^{1} \times \cdots \times S^{1}
$$

is an abstract-definable $\mathcal{C}^{p}$ manifold of dimension $m$.

Recall from [5] (p. 116) that a definable map $f: A \rightarrow R^{m}$, where $A$ is a definable subset of $R^{n}$ not necessarily open, is said to be a $C^{p}$-map if there is a definable $\mathcal{C}^{p}$ map $\tilde{f}: U \rightarrow R^{n}$, with $U$ definable and open in $R^{m}$ containing $A$, such that $\left.\widetilde{f}\right|_{A}=f$. (Then $f$ is continuous, and if $A$ is open, this gives the usual notion of $\mathcal{C}^{p}$-map on an open set.) Every inclusion $A \rightarrow R^{m}$, for $A \subseteq R^{m}$ definable, is $\mathcal{C}^{p}$. The restriction of a definable $\mathcal{C}^{p}$-map $f: A \rightarrow R^{n}$ to any definable subset of $A \subseteq R^{m}$ is a definable $\mathcal{C}^{p}$-map. If $f: A \rightarrow R^{n}$ and $g: B \rightarrow R^{q}$ are definable $\mathcal{C}^{p}$, with $A \subseteq R^{m}$ and $B \subseteq R^{n}$ definable subsets, then $g \circ f: f^{-1}(B) \rightarrow R^{q}$ is definable $\mathcal{C}^{q}$. Finally, $f=\left(f_{1}, \ldots, f_{n}\right): A \rightarrow R^{n}$ is definable $\mathcal{C}^{p}$ if and only if each function $f_{i}: A \rightarrow R$ is definable $\mathcal{C}^{p}$. 
Definition 2.7. Let $(M, \mathcal{A})$ and $(N, \mathcal{B})$ be abstract-definable $\mathcal{C}^{p}$ manifolds. A subset $A \subseteq M$ is called an abstract-definable set in $M$ if $\phi(U \cap A)$ is definable, for every chart $(U, \phi) \in \mathcal{A}$. A map $f: M \rightarrow N$ is said to be abstract-definable (resp., abstract-definable $\mathcal{C}^{p}$, an abstract-definable $\mathcal{C}^{p}$ diffeomorphism) if for any $x \in M$ and any charts $(U, \phi) \in \mathcal{A},(V, \psi) \in \mathcal{B}$ with $x \in U$ and $f(x) \in V$ the map

$$
\left.\psi \circ f \circ \phi^{-1}\right|_{\phi\left(U \cap f^{-1}(V)\right)}: \phi\left(U \cap f^{-1}(V)\right) \rightarrow \psi(f(U) \cap V)
$$

is definable (resp., a $\mathcal{C}^{p}$-map, a definable $\mathcal{C}^{p}$ diffeomorphism).

Observe from the definition of abstract-definable $\mathcal{C}^{p}$ atlas that the domain of each chart in $\mathcal{A}$ is an abstract-definable subset of $M$.

Once the notion of abstract-definable subsets has been introduced, we may now give the most useful (for our purpose) example of an abstract-definable $\mathcal{C}^{p}$ manifold, an abstract-definable open subset of a given abstract-definable $\mathcal{C}^{p}$ manifold. Because the image of a chart is definable and open, the openness as well as the abstract-definability is required to make a subset into an abstract-definable $\mathcal{C}^{p}$ manifold.

Example 2.7 (Open submanifold). Let $(M, \mathcal{A})$ be an abstract-definable $\mathcal{C}^{p}$ manifold. Every abstract-definable open subset $U$ of $M$ can be viewed in a natural way as an abstract-definable $\mathcal{C}^{p}$ manifold of the same dimension as $M$. Indeed, take

$$
\mathcal{A}_{U}:=\left\{\left.\varphi\right|_{U \cap W}: U \cap W \rightarrow \varphi(U \cap W):(W, \varphi) \in \mathcal{A}\right\}
$$

to be an abstract-definable $\mathcal{C}^{p}$ atlas on $U$. Observe that $\mathcal{A} \cup \mathcal{A}_{U}$ is an abstractdefinable $\mathcal{C}^{p}$ atlas on $M$, and consequently $\mathcal{A} \sim \mathcal{A} \cup \mathcal{A}_{U}$.

The following proposition describes some primary properties on abstract-definable sets and maps, and they will be used along the whole text without any explicit mention in most of the cases.

Proposition 2.8. Let $(M, \mathcal{A})$ and $(N, \mathcal{B})$ be abstract-definable $\mathcal{C}^{p}$ manifolds, and suppose $f: M \rightarrow N$ is an abstract-definable map.

(1) The set of all abstract-definable subsets of $M$ forms a boolean algebra under usual set operations. 
(2) For any abstract-definable subset $A$ of $M$, its topological closure $\mathrm{cl}(A)$, interior $\operatorname{int}(A)$ and boundary $\operatorname{bd}(A)$ in $M$ are also abstract-definable.

(3) For any abstract-definable subset $A$ of $M, f(A)$ is abstract-definable in $N$.

(4) For any abstract-definable subset $B$ of $N, f^{-1}(B)$ is abstract-definable in $M$.

(5) The graph $\Gamma(f)$ of $f$ is an abstract-definable subset of $M \times N$. Conversely, if $g: M \rightarrow N$ is a map whose graph $\Gamma(g)$ is an abstract-definable set in $M \times N$, then $g$ is abstract-definable.

(6) Let $g=\left(g_{1}, g_{2}\right): M \rightarrow M_{1} \times M_{2}$ be a map, where $M_{1}$ and $M_{2}$ are abstractdefinable $\mathcal{C}^{p}$ manifolds. Then, $g$ is abstract-definable $\mathcal{C}^{p}$ if and only if $g_{1}: M \rightarrow$ $M_{1}$ and $g_{2}: M \rightarrow M_{2}$ are also abstract-definable $\mathcal{C}^{p}$ maps.

(7) In the case $M, N$ are definable as well as the charts in $\mathcal{A}$ and $\mathcal{B}$, every abstract-definable subset of $M$ and all abstract-definable functions from $M$ to $N$ are definable.

Proof. (1) Given abstract-definable subsets $A$ and $B$ of $M$ and a chart $(U, \phi) \in \mathcal{A}$, it follows that $\phi(U \cap M)=\phi(U), \phi(U \cap(A \cup B))=\phi(U \cap A) \cup \phi(U \cap B)$ and $\phi(M \backslash A)=\phi(U) \backslash \phi(U \cap A)$. These equalities respectively imply $M, A \cup B$ and $M \backslash A$ are abstract-definable subsets of $M$.

(2) For any chart $(U, \phi) \in \mathcal{A}$, the subsequent equalities $\phi(U \cap \operatorname{cl}(A))=\operatorname{cl}(\phi(U \cap$ $A)) \cap \phi(U), \phi(U \cap \operatorname{int}(A))=\operatorname{int}(\phi(U \cap A))$, and $\phi(U \cap \operatorname{bd}(A))=\operatorname{bd}(\phi(U \cap A)) \cap$ $\phi(U)$ hold. Also, $\phi(U \cap A)$ is definable, by hypothesis. The result then follows by observing that the closure, interior and boundary of a definable set are also definable.

(3) Fix an arbitrary chart $(V, \psi) \in \mathcal{B}$, and note that

$$
\begin{aligned}
\psi(V \cap f(A)) & =\bigcup_{(U, \phi) \in \mathcal{A}}\left(\psi \circ f \circ \phi^{-1}\right)\left(\phi\left(f^{-1}(V) \cap A \cap U\right)\right) \\
& =\bigcup_{(U, \phi) \in \mathcal{A}}\left(\psi \circ f \circ \phi^{-1}\right)\left(\phi\left(f^{-1}(V) \cap U\right) \cap \phi(A \cap U)\right) .
\end{aligned}
$$

Since $\left.\psi \circ f \circ \phi^{-1}\right|_{\phi\left(f^{-1}(V) \cap U\right)}$ is definable and each $\phi\left(f^{-1}(V) \cap U\right) \cap \phi(A \cap U)$ is a definable subset of $\phi\left(f^{-1}(V) \cap U\right)$, the proof is finished. 
(4) We assume that $f^{-1}(B) \neq \emptyset$, otherwise it is trivially abstract-definable. Fix an arbitrary chart $(U, \phi) \in \mathcal{A}$. It is not hard to see that

$$
\phi\left(U \cap f^{-1}(B)\right)=\bigcup_{(V, \psi) \in \mathcal{B}}\left(\left.\psi \circ f \circ \phi^{-1}\right|_{\phi\left(U \cap f^{-1}(V)\right)}\right)^{-1}(\psi(f(U) \cap B \cap V))
$$

By (2), each $\phi(f(U \cap B \cap V)$ is definable, then so is its preimage $(\psi \circ f \circ$ $\left.\phi^{-1}\right)^{-1}\left(\phi(f(U \cap B \cap V))\right.$. Hence $\phi\left(U \cap f^{-1}(B)\right)$ is definable.

(5) The result is obtained easily through the fact that for an arbitrary map $h: M \rightarrow$ $N$ and any chart $(U \times V, \phi \times \psi)$ on $M \times N$,

$$
(\phi \times \psi)((U \times V) \cap \Gamma(h))=\Gamma\left(\left.\psi \circ h \circ \phi^{-1}\right|_{\phi\left(U \cap f^{-1}(V)\right)}\right) .
$$

(6) By noticing that for any $x \in M$, and for any charts $(V, \psi)$ on $M_{1}$ at $g_{1}(x)$ and $(W, \varphi)$ on $M_{2}$ at $g_{2}(x)$ we have

$\left.(\psi \times \varphi) \circ g \circ \phi^{-1}\right|_{\phi\left(U \cap g^{-1}(V \times W)\right)}=\left(\left.\psi \circ g_{1} \circ \phi^{-1}\right|_{\phi\left(U \cap g_{1}^{-1}(V)\right)},\left.\varphi \circ g_{2} \circ \phi^{-1}\right|_{\phi\left(U \cap g_{2}^{-1}(W)\right)}\right)$,

the result follows immediately.

(7) Suppose $A$ is an abstract-definable subset of $M$. Since

$$
A=\bigcup_{(U, \phi) \in \mathcal{A}}(A \cap U)=\bigcup_{(U, \phi) \in \mathcal{A}} \phi^{-1}(\phi(U \cap A))
$$

and $\phi(U \cap A), \phi^{-1}$ are definable, the set $A$ is definable. Now, if $f: M \rightarrow N$ is an abstract-definable map, then by (5) its graph $\Gamma(f)$ is an abstract-definable subset of $M \times N$, and from the first part of this item we conclude that $\Gamma(f)$ is definable.

Another quite simple but essential result is the assertion that the restriction of an abstract-definable $\mathcal{C}^{p}$ to an abstract-definable open subset remains abstractdefinable $\mathcal{C}^{p}$.

Proposition 2.9. Let $(M, \mathcal{A}),(N, \mathcal{B})$ be abstract-definable $\mathcal{C}^{p}$ manifolds, $f: M \rightarrow$ $N$ an abstract-definable $\mathcal{C}^{p}$ map, and $U$ an abstract-definable open subset of $M$. The restriction $\left.f\right|_{U}: U \rightarrow N$, with $U$ viewed as an abstract-definable $\mathcal{C}^{p}$ manifold described in Example 2.7, is also abstract-definable $\mathcal{C}^{p}$. 
Proof. Fix a point $x \in M$ and consider a chart $\left(U^{\prime}, \psi^{\prime}\right)$ on $U$ at $x$ and a chart $(W, \varphi)$ on $N$ at $f(x)$, where $U^{\prime}:=U \cap V, \psi^{\prime}:=\left.\psi\right|_{U^{\prime}}$ and $(V, \psi)$ is a chart on $M$ at $x$. Note that $\left.\varphi \circ f \circ \psi^{\prime-1}\right|_{\psi^{\prime}\left(U^{\prime} \cap f^{-1}(W)\right)}$ is the restriction of $\left.\varphi \circ f \circ \psi^{-1}\right|_{\psi\left(V \cap f^{-1}(W)\right)}$, which is definable $\mathcal{C}^{p}$ by hypothesis, to the definable subset $\psi\left(V \cap U \cap f^{-1}(W)\right)$ of $\psi\left(V \cap f^{-1}(W)\right)$. Hence, it is also a $\mathcal{C}^{p}$-map.

Proposition 2.10. Let $(M, \mathcal{A})$ be an abstract-definable $\mathcal{C}^{p}$ manifold and $A \subseteq M$. The following are equivalent.

(1) A is abstract-definable.

(2) For every $x \in A$, there is a chart $(U, \phi)$ in $\mathcal{A}$ at $x$ such that $\phi(U \cap A)$ is definable.

Proof. The direction $(1) \Rightarrow(2)$ is immediate. As for $(1) \Leftarrow(2)$, fix an arbitrary chart $(V, \psi)$ in $\mathcal{A}$. Under the assumption of $(2)$, for each $x \in A$ pick a chart $\left(U_{x}, \phi_{x}\right)$ in $\mathcal{A}$ with $x \in U_{x}$ such that $\phi_{x}\left(U_{x} \cap A\right)$ is definable. Since $\mathcal{A}$ is finite, the set of these chosen charts is finite as well. Let $J$ denote its enumeration. Then $A=\bigcup_{j \in J} U_{j} \cap A$, and consequently

$$
\psi(V \cap A)=\bigcup_{j \in J}\left(\psi \circ \phi_{j}^{-1}\right)\left(\phi_{j}\left(V \cap U_{j}\right) \cap \phi_{j}\left(U_{j} \cap A\right)\right),
$$

which shows that $\psi(V \cap A)$ is definable.

The analogue of Proposition 2.10 for maps (Proposition 3.10) also holds true, however it requires the use of a tool developed in Chapter 3.

We next verify that the $\left(\mathcal{C}^{p}\right)$ abstract-definability of sets and maps is invariant under $\sim$-equivalent atlases.

Proposition 2.11. Let $M$ and $N$ be abstract-definable $\mathcal{C}^{p}$-manifolds, and let $\mathcal{A}_{1}$, $\mathcal{A}_{2}$ be abstract-definable $\mathcal{C}^{p}$ atlases on $M$ with $\mathcal{A}_{1} \sim \mathcal{A}_{2}$, and $\mathcal{B}_{1}, \mathcal{B}_{2}$ abstractdefinable $\mathcal{C}^{p}$ atlases on $N$ such that $\mathcal{B}_{1} \sim \mathcal{B}_{2}$. Then, every subset $A \subseteq M$ abstractdefinable in $\mathcal{A}_{1}$ is abstract-definable with respect to $\mathcal{A}_{2}$, and every map $f: M \rightarrow N$ abstract-definable $\mathcal{C}^{p}$ relative to $\mathcal{A}_{1}$ and $\mathcal{B}_{1}$ is also abstract-definable $\mathcal{C}^{p}$ in $\mathcal{A}_{2}$ and $\mathcal{B}_{2}$. 
Proof. Suppose $A \subseteq M$ is an abstract-definable set with respect to $\mathcal{A}_{1}$, and fix an arbitrary chart $(V, \psi)$ in $\mathcal{A}_{2}$. From the identity

$$
\psi(V \cap A)=\bigcup_{(U, \psi) \in \mathcal{A}_{1}}\left(\psi \circ \phi^{-1}\right)(\phi(U \cap A) \cap \phi(V \cap U)),
$$

and the fact that $\psi$ is $\mathcal{C}^{p}$-compatible with $\mathcal{A}_{1}$, it follows that $A$ is also abstractdefinable with respect to $\mathcal{A}_{2}$. Now, consider a map $f: M \rightarrow N$ that is abstractdefinable $\mathcal{C}^{p}$ related to $\mathcal{A}_{1}$ and $\mathcal{B}_{1}$. Fix $x \in M$, and arbitrary charts $\left(W_{\mathcal{A}}, \varphi_{\mathcal{A}}\right) \in \mathcal{A}_{2}$ at $x$ and $\left(W_{\mathcal{B}}, \varphi_{\mathcal{B}}\right) \in \mathcal{B}_{2}$ at $f(x)$. Note that

$$
\begin{aligned}
& \varphi_{\mathcal{A}}\left(W_{\mathcal{A}} \cap f^{-1}\left(W_{\mathcal{B}}\right)\right) \\
= & \bigcup_{(U, \phi) \in \mathcal{A}_{1}} \bigcup_{(V, \psi) \in \mathcal{B}_{1}} \varphi_{\mathcal{A}}\left(W_{\mathcal{A}} \cap U \cap f^{-1}\left(W_{\mathcal{B}} \cap V\right)\right) \\
= & \bigcup_{(U, \phi) \in \mathcal{A}_{1}} \bigcup_{(V, \psi) \in \mathcal{B}_{1}}\left(\varphi_{\mathcal{A}} \circ \phi^{-1}\right)\left(\phi\left(U \cap f^{-1}\left(W_{\mathcal{B}}\right) \cap W_{\mathcal{A}}\right) \cap \phi\left(U \cap W_{\mathcal{A}} \cap f^{-1}(V)\right)\right) .
\end{aligned}
$$

Because $W_{\mathcal{B}}$ is abstract-definable in $\mathcal{B}_{2}$, the first part of the proposition then implies that $W_{\mathcal{B}}$ is abstract-definable in $\mathcal{B}_{1}$. By Lemma $2.8, f^{-1}\left(W_{\mathcal{B}}\right)$ is abstractdefinable in $\mathcal{A}_{1}$. Similarly, $W_{\mathcal{A}}$ and $f^{-1}(V)$ are both abstract-definable in $\mathcal{A}_{1}$. Hence the sets $\phi\left(U \cap f^{-1}\left(W_{\mathcal{B}}\right) \cap W_{\mathcal{A}}\right)$ and $\phi\left(U \cap W_{\mathcal{A}} \cap f^{-1}(V)\right)$ are definable, and thereby so is $\varphi_{\mathcal{A}}\left(W_{\mathcal{A}} \cap f^{-1}\left(W_{\mathcal{B}}\right)\right)$. Finally, observe that $\left.\varphi_{\mathcal{B}} \circ f \circ \varphi_{\mathcal{A}}^{-1}\right|_{\varphi_{\mathcal{A}}\left(W_{\mathcal{A}} \cap f^{-1}\left(W_{\mathcal{B}}\right)\right)}$ coincides with the $\mathcal{C}^{p}$-map $\left(\phi_{\mathcal{B}} \circ \psi\right) \circ\left(\psi \circ f \circ \phi^{-1}\right) \circ\left(\phi \circ \varphi_{\mathcal{A}}^{-1}\right)$ on $\varphi_{\mathcal{A}}\left(W_{\mathcal{A}} \cap U \cap\right.$ $\left.f^{-1}\left(W_{\mathcal{B}} \cap V\right)\right)$, therefore it is a $\mathcal{C}^{p}$-map.

Remark 2.12. Given an abstract-definable $\mathcal{C}^{p}$ manifold $(M, \mathcal{A})$ of dimension $m$, we may always assume that the range $\phi(U)$ of the charts $(U, \phi)$ in $\mathcal{A}$ are bounded open sets in $R^{m}$, because the map $\left.\tau: R^{m} \rightarrow\right]-1,1\left[^{m}\right.$ defined as

$$
\tau\left(r^{1}, \ldots, r^{m}\right):=\left(\frac{r^{1}}{\sqrt{1+\left(r^{1}\right)^{2}}}, \ldots, \frac{r^{m}}{\sqrt{1+\left(r^{m}\right)^{2}}}\right)
$$

is a semi-algebraic $\mathcal{C}^{p}$ diffeomorphism between $R^{m}$ and its image ${ }^{1}$, and the collections $\mathcal{A}$ and $\{(U, \tau \circ \phi):(U, \phi) \in \mathcal{A}\}$ are $\sim$-equivalent abstract-definable $\mathcal{C}^{p}$ atlases on $M$. By virtue of this and Theorem 1 ([31], p. 4), the image of each chart in $\mathcal{A}$ is a finite union of open cells in $R^{m}$. Since an open cell is definably $\mathcal{C}^{p}$ diffeomorphic to an open box in $R^{m}$, which in turn is definably $\mathcal{C}^{p}$ diffeomorphic to

\footnotetext{
${ }^{1}$ When $R=\mathbb{R}$, such a map is a semialgebraic real analytic diffeomorphism onto its image.
} 
$R^{m}$, we may also suppose, at our convenience, the image of any chart in $\mathcal{A}$ equals $R^{m}$.

\subsection{Some topological notions and facts}

The two ensuing results are both stated in [1], p. 115.

Lemma 2.13. Let $M$ be a set, and $\mathcal{A}$ an abstract-definable $\mathcal{C}^{p}$ atlas on $M . A$ subset $F \subseteq M$ is closed in $M$ if and only if $\phi(F \cap U)$ is closed in $\phi(U)$, for each $(U, \phi) \in \mathcal{A}$.

Proof. For any subset $F \subseteq M$ and $\operatorname{chart}(U, \phi) \in \mathcal{A}$, we have

$$
\phi(U \cap(M \backslash F))=\phi(U \backslash(U \cap F))=\phi(U) \backslash \phi(U \cap F) .
$$

Hence, $F$ is closed in $M$ if and only if $\phi(U \cap F)$ is closed in $\phi(U)$.

Corollary 2.14. Suppose $M$ is a set, and $\mathcal{A}$ is an abstract-definable $\mathcal{C}^{p}$ atlas on $M$. The manifold topology on $M$ is $T_{1}$.

Proof. For any $x \in M$ and for any chart $(U, \phi) \in \mathcal{A}$, the subset $\phi(U \cap\{x\})$ of $R^{m}$ is either the empty set or the singleton $\{\phi(x)\}$. In both cases, $\phi(U \cap\{x\})$ is a closed subset of $\phi(U)$, hence $\{x\}$ is closed in $M$, by Lemma 2.13.

The example below has been extracted from [10] (Example 2.5, p. 4), and it shows that the manifold topology is not Hausdorff. This, together with the fact that we wish the abstract-definable $\mathcal{C}^{p}$ manifolds had nice topological properties, explains why we added this separation axiom when defining the notion of abstract-definable manifold (see Definition 2.6).

Example 2.8. Let $a, b, c, d \in R$ such that $c<a<b<d$, and designate as $M$ the line segment in the plane $R^{2}$ with one point adjoined

$$
\{(x, y) \in] c, d[\times] c, d[: x=y\} \cup\{(b, a)\} .
$$

Denote by $\pi: R^{2} \rightarrow R$ the projection on the first coordinate, and set

$$
U_{1}:=M \backslash\{(b, b)\} \text { and } U_{2}:=M \backslash\{(b, a)\}
$$


(Note that $U_{1}$ and $U_{2}$ cover $M$. .) Also, denote by $\phi_{1}$, $\phi_{2}$ the bijections $\left.\pi\right|_{U_{1}}: U_{i} \rightarrow$ ]$c, d\left[\right.$ and $\left.\left.\pi\right|_{U_{2}}: U_{2} \rightarrow\right] c, d[$ respectively. Because for all subsets $U$ and $V$ of $M$ with $(b, a) \in U$ and $(b, b) \in V$ we have $b \in \phi_{1}\left(U_{1} \cap U\right) \cap \phi_{1}\left(U_{1} \cap V\right)$, it thus results the topology on $M$ induced by the atlas $\left\{\left(U_{1}, \phi_{1}\right),\left(U_{2}, \phi_{2}\right)\right\}$ is not $T_{2}$.

The next proposition is asserted in 10.1.2 ([5], p. 156) for definable spaces (Definition 10.1.1, [5], p. 156), a generalization of the notion of abstract-definable $\mathcal{C}^{0}$ manifold.

Proposition 2.15. Let $(M, \mathcal{A})$ be an abstract-definable $\mathcal{C}^{p}$ manifold. The set of abstract-definable open sets in $M$ forms a basis for the manifold topology.

Proof. We will show that any open set in $M$ is a union of abstract-definable open sets. Indeed, if $A$ is an open set in $M$, then

$$
A=\bigcup_{(U, \phi) \in \mathcal{A}} \phi^{-1}(\phi(A \cap U)),
$$

where each $\phi(U \cap A)$ is open in $R^{m}$. Since the open boxes in $R^{m}$, which are definable sets, form a basis for the product topology on $R^{m}$, we can write

$$
\phi(U \cap A)=\bigcup_{i \in I(U, \phi)} W_{(U, \phi)}^{i}
$$

with $W_{(U, \phi)}^{i}$ definable open subsets of $R^{m}$ contained in $\phi(U)$ and $I(U, \phi)$ an index set of these basic open $W_{(U, \phi)}^{i}$ covering $\phi(U \cap A)$. Hence,

$$
A=\bigcup_{(U, \phi) \in \mathcal{A}} \bigcup_{i \in I(U, \phi)} \phi^{-1}\left(W_{(U, \phi)}^{i}\right) .
$$

Recall that $\phi$ is in particular a homeomorphism and $U$ is open in $M$, therefore each $\phi^{-1}\left(W_{(U, \phi)}^{i}\right)$ is open in $M$. Also, for any $(V, \psi) \in \mathcal{A}$

$$
\psi\left(V \cap \phi^{-1}\left(W_{(U, \phi)}^{i}\right)\right)=\left(\psi \circ \phi^{-1}\right)\left(W_{(U, \phi)}^{i} \cap \phi(U \cap V)\right)
$$

is definable, thereby resulting in the conclusion that $\phi^{-1}\left(W_{(U, \phi)}^{i}\right)$ is abstract-definable in $M$.

For the remainder of the chapter, we fix an arbitrary abstract-definable $\mathcal{C}^{p}$ manifold $M$ and an abstract-definable $\mathcal{C}^{p}$ atlas $\mathcal{A}$ on $M$. 
Definition 2.16. We say that $M$ is definably regular if, for any abstractdefinable closed subset $F$ of $M$ and any point $x \in M \backslash F$, there are disjoint abstract-definable open subsets $U$ and $V$ of $M$ such that $x \in U$ and $V \subseteq F$.

Proposition 2.17. The following are equivalent.

(1) $M$ is definably regular.

(2) For any $x \in M$ and any abstract-definable open subset $U$ of $M$ with $x \in U$, there is an abstract-definable open subset $V$ of $M$ such that $x \in V \subseteq \operatorname{cl}(V) \subseteq$ $U$.

Proof. Assume that $M$ is definably regular. Let $x \in M$ and $U$ an abstractdefinable open set in $M$ containing $x$. Then, there are disjoint abstract-definable open sets $V$ and $W$ in $M$ with $x \in V$ and $(M \backslash U) \subseteq W$. Hence, $V \subseteq(M \backslash W) \subseteq U$, and since $M \backslash W$ is closed in $M, \operatorname{cl}(V) \subseteq(M \backslash W) \subseteq U$. Conversely, if $F$ is an abstract-definable closed subset of $M$ and $x$ is a point in $M \backslash F$, then applying the hypothesis for the abstract-definable open set $M \backslash F$ we obtain an abstractdefinable open subset $V \subseteq M$ containing $x$ and satisfying $\operatorname{cl}(V) \subseteq(M \backslash F)$. Hence, $M \backslash \operatorname{cl}(V)$ is an abstract-definable open set which contains $F$ and does not intersect $V$.

Definition 2.18. We say that $M$ is definably normal if, for any two abstractdefinable closed disjoint subsets $F_{1}$ and $F_{2}$ of $M$, there are disjoint abstractdefinable open subsets $U_{1}$ and $U_{2}$ such that $F_{1} \subseteq U_{1}$ and $F_{2} \subseteq U_{2}$.

Since $M$ is $T_{1}, M$ is definably regular whenever is definably normal.

Proposition 2.19. The following are equivalent.

(1) $M$ is definably normal.

(2) Given disjoint abstract-definable closed subsets $F_{1}, F_{2} \subseteq M$ there exists an abstract-definable open subset $W \subseteq M$ satisfying $F_{1} \subseteq W \subseteq \operatorname{cl}_{M}(W) \subseteq$ $\left(M \backslash F_{2}\right)$.

Proof. Assume that $M$ is definably normal, and let $F_{1}, F_{2} \subseteq M$ be disjoint abstract-definable closed subsets of $M$. Then, there exist disjoint abstract-definable 
open subsets $U, V \subseteq M$ such that $U \supseteq F_{1}$ and $V \supseteq F_{2}$. Consider the abstractdefinable open set $W:=M \backslash \operatorname{cl}(V)$ in $M$. Since $\operatorname{cl}(V) \subseteq(M \backslash U), F_{1} \subseteq W$. Also, from $F_{2} \subseteq V \subseteq \operatorname{cl}(V)$, it follows $\operatorname{cl}(W) \subseteq\left(M \backslash F_{2}\right)$. Conversely, assume (2) and let $F_{1}, F_{2} \subseteq M$ be disjoint abstract-definable closed subsets of $M$. Then, there exists an abstract-definable open set $W \subseteq M$ satisfying the condition $F_{1} \subseteq W \subseteq \mathrm{cl}_{M}(W) \subseteq\left(M \backslash F_{2}\right)$. By taking $U_{1}$ and $U_{2}$, as in Definition 2.18, to be respectively $W$ and $M \backslash \mathrm{cl}_{M}(W)$, the statement (1) follows.

The notion of definable compactness was first introduced in [20]. In a euclidean space $R^{m}$ this conception has a similar characterization ([20], Theorem 2.1, p. 772 ) to that of the non-first order property of compactness, the conjunction of boundedness and closedness.

Definition 2.20. We say that $M$ is definably compact if for every $a, b \in$ $R \cup\{-\infty,+\infty\}$ where $a<b$, and for every abstract-definable continuous map $\gamma:] a, b\left[\rightarrow M\right.$, both limits $\lim _{t \rightarrow a^{+}} \gamma(t)$ and $\lim _{t \rightarrow b^{-}} \gamma(t)$, with respect to the manifold topology, exist in $M$. We call an abstract-definable subset $K \subseteq M$ a definably compact set if for every abstract-definable continuous map $\gamma:] a, b[\rightarrow M$, with $\operatorname{Im} \gamma \subseteq K$, the limits $\lim _{t \rightarrow a^{+}} \gamma(t)$ and $\lim _{t \rightarrow b^{-}} \gamma(t)$ exist in $K$ with respect to the subspace topology on $K$. We say that $M$ is locally definably compact if every $x \in M$ has a definably compact neighborhood.

Monotonicity theorem ([5], Theorem 3.1.2, p. 43) ${ }^{2}$ yields the following.

Lemma 2.21 (Lemma 10.3, [1], p. 116). Let $a, b \in R \cup\{-\infty,+\infty\}$ with $a<b$. Then, any abstract-definable map $\gamma:] a, b[\rightarrow M$ is continuous with respect to the manifold topology on $M$, except at finitely many points.

The subsequent proposition appears in Corollary 2.8 ([10], p. 7) where the topological space is a generalization of an abstract-definable $\mathcal{C}^{0}$ manifold, namely a Hausdorff definable space (see [5], Definition 10.1.2, p. 156 or [10], Definition 2.1 , p. 3), and the background structure is an arbitrary o-minimal structure that has definable Skolem functions. That corollary is obtained by first proving that a Hausdorff, locally definably compact definable space is definably regular. Here we give a direct proof.

\footnotetext{
${ }^{2}$ Monotonicity theorem: Let $\left.f:\right] a, b[\rightarrow R$ be a definable function on the interval $] a, b[$. Then there are points $a_{1}<\ldots<a_{k}$ points in $] a, b[$ such that on each subinterval $] a_{j}, a_{j+1}\left[\right.$, with $a_{0}=a$ and $a_{k+1}=b$, the function $f$ is either constant, or strictly monotone and continuous.
} 
Proposition 2.22. If $K \subseteq M$ is a definably compact set, then $K$ is abstractdefinable closed subset of $M$.

Proof. We will show that $M \backslash K$ is open. Suppose, towards a contradiction, there is a point $x \in M \backslash K$ of which no open neighborhood is included in $M \backslash K$. Particularly, fixing a chart $(U, \phi)$ at $x$, the intersection $B(\phi(x), \epsilon) \cap \phi(U)$ is not contained in $\phi(U \cap(M \backslash K))$ for each $\epsilon>0$. By Definable Choice ([5], Theorem 6.1 .2 , p. 94), there is a definable map $\alpha:] 0, r\left[\rightarrow R^{m}\right.$ such that $\alpha(t) \in(B(\phi(x), t) \cap$ $\phi(U)) \backslash \phi(U \cap(M \backslash K))$ for all $t \in] 0, r[$. Let $\gamma:] 0, r\left[\rightarrow M\right.$ be given by $\phi^{-1} \circ \alpha$. The map $\gamma$ is abstract-definable, since for any chart $(V, \psi)$ on $M$ the composition $\psi \circ \alpha=\left(\psi \circ \phi^{-1}\right) \circ \alpha$ is a definable map. Shrinking $r$ if necessary, we may consider $\gamma$ continuous (see Lemma 2.21). Moreover, $\operatorname{Im}(\gamma) \subseteq U \cap K$ and $\lim _{t \rightarrow 0} \gamma(t)=x$. From the definable compactness of $K$ and the uniqueness of the limit (recall that $M$ is Hausdorff!), it follows that $x=\lim _{t \rightarrow 0} \gamma(t) \in K$, leading to a contradiction.

The fact that $M \backslash K$ is abstract-definable follows from the definition of definably compact set and Proposition 2.8(1).

The second part of the proposition below is contained in Proposition 2.7 ([10], p. $6)$. Our proof is rather distinct, although we also achieve the definable regularity of the abstract-definable $\left(\mathcal{C}^{p}\right)$ manifold through the local definable compactness.

Proposition 2.23. The abstract-definable $\mathcal{C}^{p}$ manifold $M$ is locally definably compact and definably regular.

Proof. Fix a point $x \in M$ and an abstract-definable open $W$ in $M$ containing $x$. Pick a chart $(U, \phi)$ on $M$ at $x$. Hence, there is an open ball $B$ with $\phi(x) \in B \subseteq$ $\operatorname{cl}(B) \subseteq \phi(U \cap W)$. Set $K:=\phi^{-1}(\operatorname{cl}(B)) \subseteq U \cap W$. Note that $x \in \operatorname{int}(K)$. Also, $K$ is abstract-definable, since for any chart $(V, \psi)$ on $M$

$$
\psi(V \cap K)=\psi\left(V \cap \phi^{-1}(\operatorname{cl}(B))\right)=\left(\psi \circ \phi^{-1}\right)(\operatorname{cl}(B) \cap \phi(U \cap V))
$$

is definable. Now, let $\gamma:] a, b[\rightarrow M$ be an abstract-definable continuous map whose image $\gamma(] a, b[)$ is contained in $K$. The map $\phi \circ \gamma:] a, b[\rightarrow \phi(U)$ is then a definable continuous curve with $\operatorname{Im}(\phi \circ \gamma) \subseteq \operatorname{cl}(B)$, and as consequence of the definable compactness of $\operatorname{cl}(B) \subseteq R^{m}$ both limits $\lim _{t \rightarrow a^{+}} \phi(\gamma(t)), \lim _{t \rightarrow b^{-}} \phi(\gamma(t))$ exist and 
belong to $\mathrm{cl}(B)$. By setting

$$
L_{1}:=\phi^{-1}\left(\lim _{t \rightarrow a^{+}} \phi(\gamma(t))\right), L_{2}:=\phi^{-1}\left(\lim _{t \rightarrow b^{-}} \phi(\gamma(t))\right) \in K
$$

and noticing that $\lim _{t \rightarrow a^{+}} \gamma(t)=L_{1}$ and $\lim _{t \rightarrow b^{-}} \gamma(t)=L_{2}$, we conclude that $K$ is a definably compact neighborhood of $x$. Consequently, $K$ is abstract-definable closed in $M$ (see Proposition 2.22), and hence

$$
x \in \phi^{-1}(B) \subseteq \operatorname{cl}\left(\phi^{-1}(B)\right) \subseteq K \subseteq W
$$

This shows the definable regularity of $M$, according to Proposition 2.17.

The following has been pointed out to us by Alessandro Berarducci in an email exchange. It can also be found in Remark 3.4 ([7], p. 9).

Proposition 2.24. The abstract-definable $C^{p}$ manifold $M$ is definably normal.

Proof. For convenience, assume without loss of generality that $|\mathcal{A}|=2$. Moreover, in view of Remark 2.12 we may suppose the images $\phi_{1}\left(U_{1}\right)$ and $\phi_{2}\left(U_{2}\right)$ of the charts in $\mathcal{A}$ are bounded. From Proposition 2.20 and the proof of Theorem 10.1 .8 ([5], p. 159) it follows that the map $h: M \rightarrow R^{1+m+1+m}$, given by

$$
h(z):= \begin{cases}\left(d_{1}(z), d_{1}(z) \phi_{1}(z), 0,0, \ldots, 0\right), & \text { if } z \in U_{1} \backslash U_{2} \\ \left(d_{1}(z), d_{1}(z) \phi_{1}(z), d_{2}(z), d_{2}(z) \phi_{2}(z)\right), & \text { if } z \in U_{1} \cap U_{2} \\ \left(0,0, \ldots, 0, d_{2}(z), d_{2}(z) \phi_{2}(z)\right), & \text { if } z \in U_{2} \backslash U_{1}\end{cases}
$$

where $d_{i}(z):=d\left(\phi_{i}(z), B_{i}\right):=\inf \left\{\left|y-\phi_{i}(z)\right|: y \in B_{i}\right\}$ and $B_{i}$ designates the definable set

$\left\{x \in R^{m}: \exists y \in \phi_{j}\left(\mathrm{bd} U_{i}\right) \forall \epsilon_{1}, \epsilon_{2}>0 \exists z \in U_{i} \cap U_{j}\left(d\left(x, \phi_{i}(z)\right)<\epsilon_{1} \wedge d\left(y, \phi_{j}(z)\right)<\epsilon_{2}\right)\right\}$

with $i, j \in\{1,2\}$ and $i \neq j$, is injective, continuous and maps $M$ homeomorphically onto the definable set $h(M)$. Let $F_{1}, F_{2} \subseteq M$ be disjoint abstract-definable closed sets. So, $h\left(F_{1}\right)$ and $h\left(F_{2}\right)$ are disjoint closed subsets of $h(M)$ and also definable, since

$$
h\left(F_{i}\right)=h\left(F_{i} \cap\left(U_{1} \backslash U_{2}\right)\right) \cup h\left(F_{i} \cap\left(U_{1} \cap U_{2}\right)\right) \cup h\left(F_{i} \cap\left(U_{2} \backslash U_{1}\right)\right)
$$


and each set in such a union is definable. By Lemma 6.3.5 ([5], p. 101), there exist disjoint definable open subsets $A_{1}$ and $A_{2}$ of $h(M)$ with $h\left(F_{i}\right) \subseteq A_{i}$. Hence, $h^{-1}\left(A_{1}\right), h^{-1}\left(A_{2}\right) \subseteq M$ are disjoint open sets containing $F_{1}$ and $F_{2}$, respectively. It remains to show that they are abstract-definable as well. To see this, first observe that $h \circ \phi_{i}^{-1}: \phi_{i}\left(U_{i}\right) \rightarrow R^{1+m+1+m}$ is a definable injective continuous map, since it is given by either

$$
\left(d\left(y, B_{i}\right), d\left(y, B_{i}\right) y, 0,0, \ldots, 0\right)
$$

or

$$
\left(d\left(y, B_{i}\right), d\left(y, B_{i}\right) y, d\left(\left(\phi_{j} \circ \phi_{i}^{-1}\right)(y), B_{j}\right), d\left(\left(\phi_{j} \circ \phi_{i}^{-1}\right)(y), B_{j}\right)\left(\phi_{j} \circ \phi_{i}^{-1}\right)(y)\right)
$$

for $y \in \phi_{i}\left(U_{i}\right)$, with $i \neq j$. Consequently, its restricted inverses $\left.\phi_{i} \circ h^{-1}\right|_{h\left(U_{1}\right)}, \phi_{i} \circ$ $\left.h^{-1}\right|_{h\left(U_{2}\right)}$ are in particular definable. Therefore, the sets

$$
\phi_{i}\left(U_{i} \cap h^{-1}\left(A_{k}\right)\right)=\left(\phi_{i} \circ h^{-1}\right)\left(h\left(U_{i}\right) \cap A_{k}\right) \cup\left(\phi_{i} \circ h^{-1}\right)\left(h\left(U_{i} \cap U_{j}\right) \cap A_{k}\right)
$$

are definable, where $i, j, k \in\{1,2\}$ and $i \neq j$.

Definition 2.25. An abstract-definable subset $S$ of $M$ is called definably connected in $M$ if there are not abstract-definable open disjoint subsets $U$ and $V$ of $M$ in such a way that $U \cap S$ and $V \cap S$ are nonempty and $S \subseteq U \cup V$. We say that $M$ is definably connected if its underlying set is definably connected in $M$. A definably connected component of a nonempty abstract-definable set $S \subseteq M$ is a maximal definably connected subset of $S$ in $M$.

The next result is an abstract-definable version of Proposition 3.2.18 ([5], p. 57).

Proposition 2.26. The abstract-definable $\mathcal{C}^{p}$ manifold $M$ has finitely many definably connected components. They form a finite partition of $M$, and consequently are open and closed in $M$.

Proof. Let $\mathcal{A}=\left\{\left(U_{i}, \phi_{i}\right): i=1, \ldots, k\right\}$. Since the subsets $\phi_{1}\left(U_{1}\right), \ldots, \phi_{i}\left(U_{i} \backslash\right.$ $\left.\bigcup_{j<i} U_{j}\right), \ldots, \phi_{k}\left(U_{k} \backslash \bigcup_{j=1}^{k-1} U_{j}\right)$ of $R^{m}$ are definable, there is a cell decomposition $\mathfrak{C}$ of $R^{m}$ partitioning them. We claim that for each $i=2, \ldots, k$ and for any $C \subseteq \phi_{1}\left(U_{1}\right), C^{\prime} \subseteq \phi_{i}\left(U_{i} \backslash \bigcup_{j<i} U_{j}\right)$ with $C, C^{\prime} \in \mathfrak{C}$, the sets $D:=\phi_{1}^{-1}(C) \subseteq U_{1}$ and $D^{\prime}:=\phi_{i}^{-1}\left(C^{\prime}\right) \subseteq U_{i} \backslash \bigcup_{j<i} U_{j}$ are definably connected in $M$. First, note that $D$ and $D^{\prime}$ are abstract-definable, inasmuch as for any chart $\left(U_{l}, \phi_{l}\right) \in \mathcal{A}$ the sets $\phi_{l}\left(U_{l} \cap D\right)$, which equals $\left(\phi_{l} \circ \phi_{1}^{-1}\right)\left(\phi_{1}\left(U_{l} \cap U_{1}\right) \cap C\right)$, and $\phi_{l}\left(U_{l} \cap D^{\prime}\right)$, which 
is identical to either $\left(\phi_{l} \circ \phi_{i}^{-1}\right)\left(\phi_{i}\left(U_{l} \cap U_{i}\right) \cap C^{\prime}\right)$ if $i \leq l$ or $\emptyset$ if $l<i$, are all definable; furthermore, if $A$ and $B$ are abstract-definable disjoint open subsets of $M$ with $D \subseteq A \cup B$, then since $\phi_{1}\left(U_{1} \cap A\right)$ and $\phi_{1}\left(U_{1} \cap B\right)$ are disjoint open definable subsets of $\phi_{1}\left(U_{1}\right)$ covering $C$, we have without loss of generality that $C \subseteq \phi_{1}\left(U_{1} \cap A\right)$, and consequently $D \subseteq U_{1} \cap A \subseteq A$. A similar argument holds for $D^{\prime}$. Therefore, we obtain a partition $\mathfrak{D}:=\left\{D_{1}, \ldots, D_{s}\right\}$ of $M$ into definably connected sets in $M$, where each element of $\mathfrak{D}$ is either of the form $\phi_{1}^{-1}(C)$ for some cell $C \in \mathfrak{C}$ included in $\phi_{1}\left(U_{1}\right)$, or of the form $\phi_{i}^{-1}\left(C^{\prime}\right)$ for some $i \in\{2, \ldots, k\}$ and a cell $C^{\prime} \in \mathfrak{C}$ included in $\phi_{i}\left(U_{i} \backslash \bigcup_{j<i} U_{j}\right)$. For each set of indices $\Lambda \subseteq\{1, \ldots, s\}$, define $D_{\Lambda}:=\bigcup_{i \in \Lambda} D_{i}$, and let $D^{*}$ be a maximal abstract-definable set with respect to the definable connectedness, among the $2^{s}-1$ nonempty sets $D_{\Lambda}$. Note that to conclude $D^{*}$ is a definably connected component of $M$, the subsequent claim suffices.

Claim 2.27. If $Y \subseteq M$ is a definably connected set in $M$ with $Y \cap D^{*} \neq \emptyset$, then $Y \subseteq D^{*}$.

Proof of the Claim. Consider the abstract-definable set

$$
D_{Y}:=\bigcup_{\substack{D_{i} \in \mathcal{Q} \\ D_{i} \cap Y \neq \emptyset}} D_{i} .
$$

Observe that $Y \subseteq D_{Y}$, since $\mathfrak{D}$ covers $M$. If $A$ and $B$ are disjoint abstract-definable open subsets of $M$ so that $D_{Y} \subseteq A \cup B$, then because $Y$ is definably connected, we may assume that $Y \subseteq A$ without loss of generality. This implies that each $D_{i}$ in $\mathfrak{D}$ with $D_{i} \cap Y \neq \emptyset$ intersects $A$, and since $D_{i}$ is definably connected in $M$, $D_{i} \subseteq A$. Hence, $D_{Y} \subseteq A$. In other words, $D_{Y}$ is definably connected. Finally, note that

$$
D^{*}, D_{Y} \supseteq D^{*} \cap D_{Y} \supseteq D^{*} \cap Y \neq \emptyset,
$$

i.e., $D^{*}$ and $D_{Y}$ have a point in common. Then, $D^{*} \cup D_{Y}$ is a set of the form $D_{\Lambda}$, for some $\Lambda \subseteq\{1, \ldots, s\}$, which is definably connected in $M$ and contains $D^{*}$. By the maximality of $D^{*}$, we get $D^{*}=D^{*} \cup D_{Y}$, and hence $Y \subseteq D_{Y} \subseteq D^{*}$.

We now draw the reader's attention to the fact that the maximal definably connected sets $D^{*}$ as above form a finite partition of $M$. Clearly, there are finitely 
many of those sets, in total. Furthermore, since $\mathfrak{D}$ covers $M$ and each of its elements is contained in such a maximal definably connected set $D^{*}$, these sets then cover $M$. Lastly, Claim 2.27 implies that the sets $D^{*}$ are pairwise disjoint.

For the ending part of the proposition statement, note that the closure in $M$ of a definably connected set in $M$ is definably connected as well, and hence by the maximality of the definably connected components these are closed subsets of $M$. Let $\left\{D_{1}^{*}, \ldots, D_{t}^{*}\right\}$ be a partition of $M$ into definably connected components. Since for each $j$

$$
D_{j}^{*}=M \backslash\left(D_{1}^{*} \cup \cdots \cup D_{j-1}^{*} \cup D_{j+1}^{*} \cup \cdots \cup D_{t}^{*}\right),
$$

it follows that $D_{j}^{*}$ is open in $M$.

A simple but quite important result concerning definable connectedness is the proposition below. Among other things, it will be used to compute the 0th de Rham cohomology group of an abstract-definable manifold (see Proposition 8.2). In order to state the proposition, we recall that a map $f: X \rightarrow Y$ between topological spaces is said to be locally constant if for each point of $X$ has a neighborhood in which $f$ is constant.

Proposition 2.28. A locally constant abstract-definable map $f: M \rightarrow N$ is constant whenever $M$ is definably connected.

Proof. It suffices to show that $f$ is constant on each definably connected component of $M$. To see this, first note that $f$ is continuous. Moreover, if $C$ is a definably connected component of $M$ and $x$ is a fixed point of $C$, then from the assumption it follows that there is an open neighborhood of $x$ in which $f$ is identical to a constant $c:=f(x) \in N$. Applying the assumption to each point in $f^{-1}(c)$ we may write $f^{-1}(c)$ as a union of open sets in $M$, hence concluding that such a set is also open in $M$. On the other hand, since $\{c\}$ is an abstract-definable closed set in $N$, $f^{-1}(c)$ is abstract-definable closed in $M$. Because $C$ is definably connected, we thus get $C \subseteq f^{-1}(c)$. 



\section{Chapter 3}

\section{Abstract-definable partitions of unity}

Our main concern in this chapter lies in proving the existence of an abstractdefinable version of partitions of unity. As is well known from the classical setting, partitions of unity play a fundamental role in the development of the theory of smooth manifolds, since they allow us passing from a local to the global context. In our case, the importance of their existence can also be appreciated when they are used to ensure the existence of abstract-definable bump functions of class $\mathcal{C}^{p}$ with $p<\infty$. This goes in the opposite direction of the classical environment in which smooth partitions of unity (subordinate to an open cover) are built explicitly out of smooth bump functions. With the purpose of constructing abstract-definable partitions of unity subordinate to a given abstract-definable $\mathcal{C}^{p}$ atlas we follow closely [11], where the author establishes among other things an abstract-definable smooth partition of unity. The meaning of "abstract-definable" in the context of [11] is the same as ours, and his construction perfectly fits in our setting. However, in order to obtain such a partition, A. Fischer develops some results on smoothing of continuous functions that are definable in an o-minimal expansion of the real field in which the exponential function is definable, and which admits smooth cell decomposition. The question of whether similar theorems on approximation of definable functions are also available in the general framework of an o-minimal expansion of a real closed field is then answered affirmatively in a more recent paper [29] by A. Thamrongthanyalak as part of his Phd Thesis, keeping Fischer's strategy handy. The latter part of the chapter is devoted to applications of abstractdefinable $\mathcal{C}^{p}$ partitions of unity such as an abstract-definable $\mathcal{C}^{p}$ version of Urysohn 
lemma, the already mentioned existence of abstract-definable $\mathcal{C}^{p}$ bump functions supported in abstract-definable open sets, among others.

\subsection{Abstract-definable partitions of unity}

In preparation for the proof of the main theorem of this chapter (Theorem 3.4), we state and prove a lemma on dimension of definable sets, implicitly contained in Exercises 7.3 .3 ([5], p. 116).

Lemma 3.1. Let $f: U \rightarrow R$ be a definable function, where $U$ is a definable open set in $R^{m}$, and denote by $C$ the definable set $\left\{x \in U: f\right.$ is not of class $\mathcal{C}^{p}$ at $\left.x\right\}$. Then, $\operatorname{dim}\left(\operatorname{cl}_{U}(C)\right)<\operatorname{dim}(U)$.

Proof. Firstly, observe that $\operatorname{dim}\left(\operatorname{cl}_{U}(C)\right) \leq \operatorname{dim}(\operatorname{cl}(C))=\operatorname{dim}(C)$. If we show that $\operatorname{dim}(C)<\operatorname{dim}(U)$, the lemma then follows. By $\mathcal{C}^{p}$ cell decomposition, there are $\mathcal{C}^{p}$-cells $E_{1}, \ldots, E_{k}$ in $R^{m}$ such that $\left\{E_{1}, \ldots, E_{k}\right\}$ partitions $U$ and $\left.f\right|_{E_{i}}: E_{i} \rightarrow R$ is $\mathcal{C}^{p}$, for each $i \in\{1, \ldots, k\}$. Consequently,

$$
C \subseteq \bigcup_{i=1}^{k} \operatorname{bd}_{U}\left(E_{i}\right)
$$

From Proposition 4.1.3(iii)([5], p. 64) $)^{1}$, it follows that

$$
\operatorname{dim}\left(\bigcup_{i=1}^{k} \operatorname{bd}_{U}\left(E_{i}\right)\right)=\max \left\{\operatorname{dim}\left(\operatorname{bd}_{U}\left(E_{i}\right)\right): i=1, \ldots, k\right\} .
$$

But, for each $i$, we have $\operatorname{dim}\left(\operatorname{bd}_{U}\left(E_{i}\right)\right) \leq \operatorname{dim}\left(\operatorname{bd}\left(E_{i}\right)\right)$ and $\operatorname{dim}\left(\operatorname{bd}\left(E_{i}\right)\right)<m$, by Corollary 4.1 .10 ([5], p. 68) $)^{2}$. Since $U$ is open in $R^{m}, \operatorname{dim}(U)=m$. Putting the pieces together, we have the inequalities

$$
\operatorname{dim}(C) \leq \operatorname{dim}\left(\bigcup_{i=1}^{k} \operatorname{bd}_{U}\left(E_{i}\right)\right)<\operatorname{dim}(U)
$$

\footnotetext{
${ }^{1}$ Proposition 4.1.3(iii). If $X, Y \subseteq R^{m}$ are definable, then $\operatorname{dim}(X \cup Y)=\max \{\operatorname{dim} X$, $\operatorname{dim} Y\}$.

${ }^{2}$ Corollary 4.1.10. Let $S \subseteq R^{m}$ be definable. Then $\operatorname{dim}(\operatorname{bd}(S))<m$, where $\operatorname{bd}(S):=\operatorname{cl}(S)-$ $\operatorname{int}(S)$ is the topological boundary of the set $S$ in $R^{m}$.
} 
Starting from the Generalized Lojasiewicz Inequality ([6], Theorem C.14) and using a stratification of definable sets where the functions involved in the strata have bounded gradient (Theorem 3.7, [29], p. 9), A. Thamrongthanyalak attains a deep and fruitful result on definable smoothing of definable continuous functions, stated as follows.

Theorem 3.2 (Theorem 1.1, [29], p. 2). Let $f: U \rightarrow R$ be a definable continuous function, with $U$ open in $R^{n}$. Let $Z$ be a definable closed subset of $U$ such that $\operatorname{dim}(Z)<\operatorname{dim}(U)$, and $\left.f\right|_{(U \backslash Z)}$ is $\mathcal{C}^{p}$, where $p \geq 1$. Let $\left.\epsilon: U \rightarrow\right] 0, \infty[$ be a definable continuous function. Then, for any definable neighborhood $V$ of $Z$ in $U$, there is a definable $\mathcal{C}^{p}$ function $g: U \rightarrow R$ such that

(1) $|g(x)-f(x)|<\epsilon(x)$, for every $x \in U$;

(2) $g=f$ outside $V$.

The subsequent lemma, a weak definable $\mathcal{C}^{p}$ version of Urysohn's lemma, is Corollary 1.2 in [11] (p. 497). The proof here is an adjustment from that of Corollary 1.2 to our setting.

Lemma 3.3. Let $U \subseteq R^{m}$ be a definable open set, and let $A, B \subseteq U$ be definable disjoint sets which are closed in $U$. Then, there is a definable $\mathcal{C}^{p}$ function $f: U \rightarrow$ $R$ such that $A \subseteq\{f=1\}$ and $B \subseteq\{f=0\}$.

Proof. Since $U$ is definably normal, there are disjoint definable open neighborhoods $V_{A}^{\prime}$ and $V_{B}^{\prime}$ in $U$ such that $A \subseteq V_{A}^{\prime}$ and $B \subseteq V_{B}^{\prime}$. Again, by the definable normality of $U$, there are definable open sets $V_{A}$ and $V_{B}$ in $U$ satisfying

$$
A \subseteq V_{A} \subseteq \operatorname{cl}_{U}\left(V_{A}\right) \subseteq V_{A}^{\prime} \text { and } B \subseteq V_{B} \subseteq \operatorname{cl}_{U}\left(V_{B}\right) \subseteq V_{B}^{\prime}
$$

Hence, $\operatorname{cl}_{U}\left(V_{A}\right) \cap \mathrm{cl}_{U}\left(V_{B}\right)=\emptyset$. By Lemma $6.3 .8([5], \text { p. } 102)^{3}$, there is a definable continuous function $g: U \rightarrow R$ such that $0 \leq g \leq 1, g^{-1}(1)=\operatorname{cl}_{U}\left(V_{A}\right)$, and $g^{-1}(0)=\mathrm{cl}_{U}\left(V_{B}\right)$. Let $C$ be the definable set of points in $U$ at which $g$ is not $\mathcal{C}^{p}$, that is,

$$
C:=\left\{x \in U: g \text { is not } \mathcal{C}^{p} \text { at } x\right\} .
$$

\footnotetext{
${ }^{3}$ Lemma 6.3.8. Suppose $(R,<, \mathcal{S})$ expands an ordered real closed field. Let $A_{0}$ and $A_{1}$ be disjoint definable closed subsets of a definable set $B \subseteq R^{m}$. Then there is a continuous definable function $f: B \rightarrow[0,1]$ with $f^{-1}(0)=A_{0}$ and $f^{-1}(1)=A_{1}$.
} 
Since $C$ is contained in the definable closed set $U \backslash\left(V_{A} \cup V_{B}\right), \mathrm{cl}_{U}(C) \cap\left(V_{A} \cup V_{B}\right)=\emptyset$. Particularly, we have

$$
\mathrm{cl}_{U}(C) \cap(A \cup B)=\emptyset .
$$

Then from Lemma 3.1, it follows that $\operatorname{dim}\left(\operatorname{cl}_{U}(C)\right)<\operatorname{dim}(U)$. Hence, by Theorem 3.2, there is a definable $\mathcal{C}^{p}$ function $f: U \rightarrow R$ such that $f=g$ in $A \cup B$.

For the remainder of this chapter, we fix an arbitrary abstract-definable $\mathcal{C}^{p}$-manifold $M$ of dimension $m$, and an arbitrary atlas $\mathcal{A}:=\left\{\phi_{i}: U_{i} \rightarrow \phi_{i}\left(U_{i}\right)\right\}_{i \in I}$ with $I:=\{1, \ldots, l\}$ on $M$.

As we remarked previously in distinct occasions, to prove the theorem below, we have used the same tecnique as A. Fischer has employed when proving Lemma 4.6 ([11], p. 508). There is a substantial modification, though, in the argument of the construction of the functions $\psi_{i}$ (here we are adopting the same notation as in the paper [11]) motivated by the fact that we have not succeeded in verifying the assertion " $\operatorname{supp}\left(\psi_{i}\right) \subseteq U_{i}$ " in his proof of Lemma 4.6 ([11], p. 508). Roughly speaking, the point is that A. Fischer separates certain sets involved in the construction of the functions $\psi_{i}$ in the images $\phi_{i}\left(U_{i}\right)$, which raises the possibility of the support of $\psi_{i}$ to touch the boundary of $U_{i}$ after we get back to the manifold $M$ via its charts. We overcome this problem by first covering the boundaries $\operatorname{bd}\left(U_{i}\right)$ of the domains of the charts $\left(U_{i}, \phi_{i}\right)$ with open sets, for the sake of the normality of $M$, in such a way that the closure of the set $\left\{\psi_{i} \neq 0\right\}$ does not reach the corresponding boundary $\operatorname{bd}\left(U_{i}\right)$.

Theorem 3.4 (Existence of abstract-definable partitions of unity). For each $i \in I$, there exists an abstract-definable $\mathcal{C}^{p}$ function $\varphi_{i}: M \rightarrow R$ such that:

(a) $\varphi_{i} \geq 0$;

(b) $\operatorname{supp}\left(\varphi_{i}\right) \subseteq U_{i}$;

(c) $\sum_{i \in I} \varphi_{i}=1$.

Recall that $\operatorname{supp}\left(\varphi_{i}\right)$ denotes the support of the function $\varphi_{i}$, that is, the closure of the set $\left\{x \in M: \varphi_{i}(x) \neq 0\right\}$ in $M$. The collection $\left\{\varphi_{i}\right\}_{i \in I}$ is called an abstractdefinable $\mathcal{C}^{p}$ partition of unity subordinate to $\left\{U_{i}\right\}_{i \in I}$.

Proof. For simplicity reasons, we assume without loss of generality that $I=\{1,2\}$. 
Claim 3.5. There exist abstract-definable $\mathcal{C}^{p}$ functions $\psi_{1}, \psi_{2}: M \rightarrow R$ such that $\psi_{i} \geq 0, \operatorname{supp}\left(\psi_{i}\right) \subseteq U_{i}$, and $M=\psi_{1}^{-1}(] 0,+\infty[) \cup \psi_{2}^{-1}(] 0,+\infty[)$.

Proof of the Claim. Let $V_{1}:=U_{1}-U_{2}$. Note that $V_{1}$ is an abstract-definable closed subset of $M$ which does not intersect the abstract-definable closed subset $\operatorname{bd}\left(U_{1}\right)$. Hence, there are disjoint abstract-definable open sets $\Omega_{1}$ and $\Omega_{2}$ in $M$ such that $V_{1} \subseteq \Omega_{1}$ and $\operatorname{bd}\left(U_{1}\right) \subseteq \Omega_{2}$ (recall that $M$ is definably normal). Let $W_{1}:=$ $\operatorname{cl}\left(\Omega_{2}\right) \cap U_{1}$. Since $\Omega_{1} \cap \operatorname{cl}\left(\Omega_{2}\right)=\emptyset$, the (abstract-definable) closed subsets $V_{1}$ and $W_{1}$ of $U_{1}$ are disjoint. Consequently, $\phi_{1}\left(V_{1}\right)$ and $\phi\left(W_{1}\right)$ are disjoint definable closed subsets of $\phi_{1}\left(U_{1}\right)$. By Lemma 3.3, there is a definable $\mathcal{C}^{p}$ function $f_{1}: \phi_{1}\left(U_{1}\right) \rightarrow R$ such that $\phi_{1}\left(V_{1}\right) \subseteq\left\{f_{1}=1\right\}$ and $\phi_{1}\left(W_{1}\right) \subseteq\left\{f_{1}=0\right\}$. Squaring if necessary, we may assume that $f_{1} \geq 0$. Take $\psi_{1}: M \rightarrow R$ to be the function given by

$$
\psi_{1}:= \begin{cases}f_{1} \circ \phi_{1}, & \text { on } U_{1} \\ 0, & \text { on } M \backslash U_{1}\end{cases}
$$

Clearly, $\psi_{1}$ is an abstract-definable nonnegative function. In order to obtain the inclusion $\operatorname{supp}\left(\psi_{1}\right) \subseteq U_{1}$, it suffices to prove that $\operatorname{cl}\left(\phi_{1}^{-1}\left(\left\{f_{1} \neq 0\right\}\right)\right)$ does not intersect $\operatorname{bd}\left(U_{1}\right)$, once $\left\{\psi_{1} \neq 0\right\}=\phi_{1}^{-1}\left(\left\{f_{1} \neq 0\right\}\right)$ and $\operatorname{cl}\left(\phi_{1}^{-1}\left(\left\{f_{1} \neq 0\right\}\right)\right)$ is contained in $U_{1} \cup \operatorname{bd}\left(U_{1}\right)$. Indeed, from the inclusions

$$
\begin{aligned}
\phi_{1}^{-1}\left(\left\{f_{1} \neq 0\right\}\right)=\left(U_{1} \backslash \phi_{1}^{-1}\left(\left\{f_{1}=0\right\}\right)\right) \subseteq\left(U_{1} \backslash W_{1}\right) & =\left(U_{1} \backslash \operatorname{cl}\left(\Omega_{2}\right)\right) \\
& \subseteq\left(U_{1} \backslash \Omega_{2}\right) \subseteq\left(M \backslash \Omega_{2}\right)
\end{aligned}
$$

it follows that $\operatorname{cl}\left(\phi_{1}^{-1}\left(\left\{f_{1} \neq 0\right\}\right)\right) \cap \Omega_{2}=\emptyset$, and in particular the intersection of the sets $\operatorname{cl}\left(\phi_{1}^{-1}\left(\left\{f_{1} \neq 0\right\}\right)\right)$ and $\operatorname{bd}\left(U_{1}\right)$ is empty. Lastly, the function $\psi_{1}$ is $\mathcal{C}^{p}$, because $\psi_{1}$ is the product of two $\mathcal{C}^{p}$ functions on $U_{1}$, and for any $x \in\left(M \backslash U_{1}\right) \subseteq$ $\left(M \backslash \operatorname{supp}\left(\psi_{1}\right)\right)$ there is an (abstract-definable) open neighborhood of $x$ in which $\psi_{1}$ vanishes.

Proceeding in a similar way for $V_{2}:=U_{2} \backslash \psi_{1}^{-1}(] 0,+\infty[)$ and $W_{2}:=\operatorname{cl}\left(\Theta_{2}\right) \cap U_{2}$, where $\Theta_{2}$ is an abstract-definable open subset of $M$ containing $\operatorname{bd}\left(U_{2}\right)$ whose existence is ensured by the definable normality of $M$, we may construct an abstractdefinable $\mathcal{C}^{p}$ nonnegative function $\psi_{2}: M \rightarrow R$ satisfying $\operatorname{supp}\left(\psi_{2}\right) \subseteq U_{2}$.

Finally, note that the sets $\left\{\psi_{1}>0\right\},\left\{\psi_{2}>0\right\}$ cover $M$, since otherwise any point outside the union $\bigcup_{i=1}^{2} \psi_{i}\left(\left[0,+\infty[)\right.\right.$ would not lie in $U_{1} \cup U_{2}$, by the construction of the functions $\psi_{i}$. 
In view of Claim 3.5 we define $\varphi_{i}: M \rightarrow R$ as

$$
\varphi_{i}:=\frac{\psi_{i}}{\psi_{1}+\psi_{2}}, i=1,2
$$

Observe that the function $\varphi_{i}$ is well-defined, that is, the denominator of (3.2) never vanishes. Moreover, $\varphi_{i} \geq 0$, and $\varphi_{i}=0$ if and only if $\psi_{i}=0$. Hence, $\operatorname{supp}\left(\varphi_{i}\right)=\operatorname{supp}\left(\psi_{i}\right) \subseteq U_{i}$. At last,

$$
\sum_{i=1}^{2} \varphi_{i}=\sum_{i=1}^{2}\left(\frac{\psi_{i}}{\psi_{1}+\psi_{2}}\right)=1
$$

\subsection{Abstract-definable bump functions and other consequences}

Now we derive some results from the existence of abstract-definable $\mathcal{C}^{p}$ partitions of unity whose analogues in the standard setting are widely known.

Proposition 3.6. Let $\{V, W\}$ be an abstract-definable open cover of $M$. Then, there are abstract-definable $\mathcal{C}^{p}$ functions $f_{V}, f_{W}: M \rightarrow R$ such that:

(1) $f_{V}, f_{W} \geq 0$

(2) $\operatorname{supp}\left(f_{V}\right) \subseteq V$ and $\operatorname{supp}\left(f_{W}\right) \subseteq W$;

(3) $f_{V}+f_{W}=1$.

Proof. Consider the following abstract-definable $\mathcal{C}^{p}$ maps

$$
\phi_{i}^{V}:=\left.\phi_{i}\right|_{V_{i}} \text { and } \phi_{i}^{W}:=\left.\phi_{i}\right|_{W_{i}}
$$

where $V_{i}:=U_{i} \cap V$, and $W_{i}:=U_{i} \cap W$, for each $i \in I$. Then, the pair

$$
\left(M,\left\{\phi_{i}^{V}: V_{i} \rightarrow \phi_{i}\left(V_{i}\right), \phi_{i}^{W}: W_{i} \rightarrow \phi_{i}\left(W_{i}\right)\right\}_{i \in I}\right)
$$

is an abstract-definable $\mathcal{C}^{p}$ atlas on $M$ which is $\sim$-equivalent to $\mathcal{A}$. Applying Theorem 3.4 to this atlas, we obtain abstract-definable $\mathcal{C}^{p}$ nonnegative functions 
$\varphi_{i}^{V}: M \rightarrow R, \varphi_{i}^{W}: M \rightarrow R$ with $i \in I$ satisfying the conditions

$$
\operatorname{supp}\left(\varphi_{i}^{V}\right) \subseteq V_{i}, \operatorname{supp}\left(\varphi_{i}^{W}\right) \subseteq W_{i}, \text { and } \sum_{i \in I} \varphi_{i}^{V}+\sum_{i \in I} \varphi_{i}^{W}=1
$$

Define $f_{V}: M \rightarrow R$ and $f_{W}: M \rightarrow R$ as

$$
f_{V}:=\sum_{i \in I} \varphi_{i}^{V}, \text { and } f_{W}:=\sum_{i \in I} \varphi_{i}^{W}
$$

respectively. It is readily seen that $f_{V}$ and $f_{W}$ are abstract-definable $\mathcal{C}^{p}$ nonnegative functions. Moreover,

$$
\operatorname{supp}\left(f_{V}\right)=\bigcup_{i \in I} \operatorname{supp}\left(\varphi_{i}^{V}\right)
$$

since $f_{V}(x) \neq 0$ if, and only if, $\varphi_{i}^{V}(x) \neq 0$, for some $i \in I$. Hence,

$$
\operatorname{supp}\left(f_{V}\right) \subseteq \bigcup_{i \in I} V_{i}=V
$$

Similarly, $\operatorname{supp}\left(f_{W}\right) \subseteq W$. As an immediate consequence of the construction of $f_{V}$ and $f_{W}$, we finally get

$$
f_{V}+f_{W}=1
$$

Proposition 3.7 (Abstract-definable Urysohn's lemma). Let $A$ and $B$ be disjoint abstract-definable closed sets in $M$. Then, there exists an abstract-definable $\mathcal{C}^{p}$ nonnegative function $f: M \rightarrow R$ which is identically 1 on $A$, and identically 0 on $B$.

Proof. Apply Proposition 3.6 to the abstract-definable open cover $\{V, W\}$ of $M$, where $V:=M \backslash A$, and $W:=M \backslash B$, to obtain abstract-definable $\mathcal{C}^{p}$ nonnegative functions $f_{V}, f_{W}: M \rightarrow R$ such that $\operatorname{supp}\left(f_{V}\right) \subseteq V, \operatorname{supp}\left(f_{W}\right) \subseteq W$, and $f_{V}+$ $f_{W}=1$. Then, by taking $f: M \rightarrow R$ to be $f_{W}$, the result follows.

Proposition 3.8. Let $F$ be an abstract-definable closed set in $M$, and $U$ an abstract-definable open set in $M$ containing $F$. Then, there exists an abstractdefinable $\mathcal{C}^{p}$ function $\rho: M \rightarrow R$ so that

(1) $0 \leq \rho \leq 1$; 
(2) $\left.\rho\right|_{F}=1$;

(3) $\operatorname{supp}(\rho) \subseteq U$.

Proof. Let $\{M \backslash F, U\}$ be an abstract-definable open cover of $M$. By Proposition 3.6, there are abstract-definable $\mathcal{C}^{p}$ nonnegative functions $f, g: M \rightarrow R$ which have the properties

$$
\operatorname{supp}(f) \subseteq M \backslash F, \operatorname{supp}(g) \subseteq U, \text { and } f+g=1
$$

Put $\rho:=g$. Hence, $\rho$ satisfies the conditions (1), (2), and (3) above.

Corollary 3.9 (Existence of abstract-definable bump functions). For any abstractdefinable open subset $U \subseteq M$, there is an abstract-definable $\mathcal{C}^{p}$ nonnegative function $\rho: M \rightarrow R$ such that

(1) $\left.\rho\right|_{V}=1$, for some abstract-definable open set $V \subseteq U$;

(2) $\operatorname{supp}(\rho) \subseteq U$.

The function $\rho$ is called an abstract-definable $\mathcal{C}^{p}$ bump function supported in $U$.

Proof. Fix a point $x$ in $U$. Since $M$ is definably regular (see Proposition 2.23), by Proposition 2.17 there is an abstract-definable open set $V$ satisfying

$$
x \in V \subseteq \operatorname{cl}(V) \subseteq U .
$$

By applying Proposition 3.8 to $\operatorname{cl}(V)$, the desired function $\rho$ is immediately obtained.

The following proposition is the analogue for maps of Proposition 2.10. It will be used specifically in the proofs of Propositions 6.3 and 6.13 .

Proposition 3.10. Let $f: M \rightarrow N$ be a map, where $(N, \mathcal{B})$ is an abstract-definable $\mathcal{C}^{p}$ manifold. The following are equivalent.

(1) $f$ is abstract-definable $\mathcal{C}^{p}$. 
(2) For each $x \in M$, there are charts $(U, \phi) \in \mathcal{A}$ at $x$ and $(V, \psi) \in \mathcal{B}$ at $f(x)$ such that $\left.\psi \circ f \circ \phi^{-1}\right|_{\phi\left(U \cap f^{-1}(V)\right)}$ is a $\mathcal{C}^{p}$-map.

Proof. Under the assumption of (1), we immediately obtain (2). Conversely, assume (2), and let $x$ be a point in $M$ and $(U, \phi) \in \mathcal{A},(V, \psi) \in \mathcal{B}$ arbitrary charts at $x$ and $f(x)$ respectively. We must prove that the restriction of $\psi \circ f \circ \phi^{-1}$ to $\phi\left(U \cap f^{-1}(V)\right)$ is a $\mathcal{C}^{p}$-map. This will be done first by showing that the concerned restricted map is definable, and then it is extendable to a definable map of class $\mathcal{C}^{p}$ defined on an open definable set. For each $z \in U \cap f^{-1}(V)$, pick a chart $\left(U_{z}, \phi_{z}\right) \in \mathcal{A}$ with $z \in U_{z}$ and a chart $\left(V_{z}, \psi_{z}\right) \in \mathcal{B}$ with $f(z) \in V_{z}$, such that $\left.\psi_{z} \circ f \circ \phi_{z}^{-1}\right|_{\phi_{z}\left(U_{z} \cap f^{-1}\left(V_{z}\right)\right)}$ is a $\mathcal{C}^{p}$-map. Since the set of these chosen charts is contained in $\mathcal{A} \cup \mathcal{B}, U \cap f^{-1}(V)$ can be expressed as a finite union

$$
U \cap f^{-1}(V)=\bigcup_{\alpha \in \Lambda}\left(U \cap f^{-1}(V)\right) \cap\left(U_{\alpha} \cap f^{-1}\left(V_{\alpha}\right)\right)
$$

where $\Lambda$ is an enumeration of this set of chosen charts. Consequently,

$$
\phi\left(U \cap f^{-1}(V)\right)=\bigcup_{\alpha \in \Lambda} \phi\left(U \cap f^{-1}(V) \cap U_{\alpha} \cap f^{-1}\left(V_{\alpha}\right)\right) .
$$

Note that on each definable set $\phi\left(U \cap f^{-1}(V) \cap U_{\alpha} \cap f^{-1}\left(V_{\alpha}\right)\right)$ the map $\psi \circ f \circ \phi^{-1}$ equals the $\mathcal{C}^{p}$-map $\left(\psi \circ \psi_{\alpha}^{-1}\right) \circ\left(\psi_{\alpha} \circ f \circ \phi_{\alpha}^{-1}\right) \circ\left(\phi_{\alpha} \circ \phi^{-1}\right)$. Therefore, $\psi \circ f \circ$ $\left.\phi^{-1}\right|_{\phi\left(U \cap f^{-1}(V) \cap U_{\alpha} \cap f^{-1}\left(V_{\alpha}\right)\right)}$ is a $\mathcal{C}^{p}$-map and the restriction $\left.\psi \circ f \circ \phi^{-1}\right|_{\phi\left(U \cap f^{-1}(V)\right)}$ is definable. Now put

$$
g_{\alpha}:=\left.\psi \circ f \circ \phi^{-1}\right|_{\phi\left(U \cap f^{-1}(V) \cap U_{\alpha} \cap f^{-1}\left(V_{\alpha}\right)\right)} .
$$

By the definition of $\mathcal{C}^{p}$-map, for each $\alpha$ there is a definable $\mathcal{C}^{p}$ map $\widetilde{g}_{\alpha}: W_{\alpha} \rightarrow R^{m}$, with $W_{\alpha}$ definable open subset of $R^{m}$ containing the definable set $\phi\left(U \cap f^{-1}(V) \cap\right.$ $\left.U_{\alpha} \cap f^{-1}\left(V_{\alpha}\right)\right)$, which extends $g_{\alpha}$. Set $W:=\bigcup_{\alpha \in \Lambda} W_{\alpha}$. Observe that $W$ is a definable open set in $R^{m}$ containing each set $\phi\left(U \cap f^{-1}(V) \cap U_{\alpha} \cap f^{-1}\left(V_{\alpha}\right)\right)$. Also, $\left(W,\left\{\operatorname{id}_{W_{\alpha}}: W_{\alpha} \rightarrow W_{\alpha}\right\}_{\alpha \in \Lambda}\right)$ is an abstract-definable $\mathcal{C}^{p}$ manifold. Theorem 3.4 thus ensures the existence of abstract-definable $\mathcal{C}^{p}$ functions $\varphi_{\alpha}: W \rightarrow R, \alpha \in \Lambda$, satisfying the conditions: $\varphi_{\alpha} \geq 0, \operatorname{supp}\left(\varphi_{\alpha}\right) \subseteq W_{\alpha}$, and $\sum_{\alpha \in \Lambda} \varphi_{\alpha}=1$. Because the underlying set $W$ and the charts $\operatorname{id}_{W_{\alpha}}$ are all definable, the functions $\varphi_{\alpha}$ are 
also definable. Define $\widetilde{g}: W \rightarrow R^{n}$ as

$$
\widetilde{g}(x):=\sum_{\alpha \in \Lambda} \varphi_{\alpha}(x) \cdot \widetilde{g}_{\alpha}(x)
$$

It is not hard to see that $\widetilde{g}$ is definable $\mathcal{C}^{p}$. Moreover, for every $y \in \phi\left(U \cap f^{-1}(V)\right)$

$$
\begin{aligned}
\widetilde{g}(y)=\sum_{\alpha \in \Lambda} \varphi_{\alpha}(y) \cdot \widetilde{g}_{\alpha}(y) & =\sum_{\alpha \in \Lambda^{\prime}} \varphi_{\alpha}(y) \cdot\left(\psi \circ f \circ \phi^{-1}\right)(y) \\
& =\left(\sum_{\alpha \in \Lambda^{\prime}} \varphi_{\alpha}(y)\right) \cdot\left(\psi \circ f \circ \phi^{-1}\right)(y) \\
& =\left(\psi \circ f \circ \phi^{-1}\right)(y),
\end{aligned}
$$

where $\Lambda^{\prime}:=\left\{\alpha \in \Lambda: y \in W_{\alpha}\right\}$. In summary, $\left.\psi \circ f \circ \phi^{-1}\right|_{\phi\left(U \cap f^{-1}(V)\right)}$ is extended to a definable $\mathcal{C}^{p}$ map defined on a definable open set. This finishes the proof. 


\section{Chapter 4}

\section{The tangent and cotangent bundles}

In this chapter, we introduce the notion of the tangent space at a point to an abstract-definable manifold. As in the classical setting, this allows us to define concepts like differential and partial derivatives of an abstract-definable $\mathcal{C}^{p}$ map, and then the tangent bundle of an abstract-definable manifold, which is an abstractdefinable manifold as well. In the second part of the chapter, we introduce the cotangent bundle of an abstract-definable manifold, and similarly show that it can be made into an abstract-definable manifold.

Throughout this chapter, unless otherwise stated, $(M, \mathcal{A})$ and $(N, \mathcal{B})$ denote abstractdefinable definable $\mathcal{C}^{p}$ manifolds of dimensions $m$ and $n$, respectively.

\subsection{The tangent bundle}

Our approach to the construction of the tangent space is the same as in chapter 9 ([19], p. 65).

Fix $x \in M$, and denote by $\mathcal{C}^{p}(M, x)$ the set of all abstract-definable $\mathcal{C}^{p}$ curves on an open interval $I \subseteq R$ such that $0 \in I$ and $\gamma(0)=x$. We say that $\gamma_{1}, \gamma_{2} \in \mathcal{C}^{p}(M, x)$ are tangent at 0 if there is a chart $(U, \phi)$ on $M$ with $x \in U$ such that

$$
\left(\phi \circ \gamma_{1}\right)^{\prime}(0)=\left(\phi \circ \gamma_{2}\right)^{\prime}(0)
$$

(It is implied that $\phi \circ \gamma_{1}$ and $\phi \circ \gamma_{2}$ are defined on definable open subsets of $R$ containing 0 , for instance, on $\gamma_{1}^{-1}(U)$ and $\gamma_{2}^{-1}(U)$, respectively.) Observe that, by 
virtue of the chain rule for definable maps, we can replace the condition "for some chart on $M$ at $x$ " with "for any chart on $M$ at $x$ " in the definition of tangent curves at 0 above.

Define on $\mathcal{C}^{p}(M, x)$ the relation

$$
\gamma_{1} \sim_{c} \gamma_{2} \stackrel{\text { def }}{\Leftrightarrow} \gamma_{1} \text { and } \gamma_{2} \text { are tangent at } 0
$$

It is not hard to see that $\sim_{c}$ is an equivalence relation. We denote by $T_{x} M$ the set $\mathcal{C}^{p}(M, x) / \sim_{c}$.

Lemma 4.1. Let $x \in M$. For every $\operatorname{chart~}(U, \phi)$ on $M$ with $x \in U$, the map $\bar{\phi}_{x}: T_{x} M \rightarrow R^{m}$ given by

$$
[\gamma] \mapsto(\phi \circ \gamma)^{\prime}(0)
$$

is bijective.

Proof. The fact that $\bar{\phi}_{x}$ is well-defined and injective follows from the definition of $\sim_{c}$. As for the surjectivity of $\bar{\phi}_{x}$, let $a \in R^{m}$ be an arbitrary element. Since the map $t \mapsto \phi(x)+t a$ is continuous at $t=0$, there is $\epsilon>0$ such that $\phi(x)+t a \in \phi(U)$ for all $t \in]-\epsilon, \epsilon\left[\right.$. Now, take $\gamma$ as the abstract-definable $\mathcal{C}^{p}$ curve

$$
\left.t \mapsto \phi^{-1}(\phi(x)+t a):\right]-\epsilon, \epsilon[\rightarrow M
$$

Then, $\gamma \in \mathcal{C}^{p}(M, x)$ and $a=(\phi \circ \gamma)^{\prime}(0)=\bar{\phi}_{x}([\gamma])$.

As a consequence of Lemma 4.1, there is a unique $R$-vector space structure on $T_{x} M$ which makes the bijection $\bar{\phi}_{x}$ above into a linear isomorphism, namely: for all $v, w \in T_{x} M$ and $\lambda \in R$, we set up

$$
v+w:=\bar{\phi}_{x}^{-1}\left(\bar{\phi}_{x}(v)+\bar{\phi}_{x}(w)\right) \text { and } \lambda \cdot v:=\bar{\phi}_{x}^{-1}\left(\lambda \cdot \bar{\phi}_{x}(v)\right)
$$

If $(V, \psi)$ is another chart on $M$ at $x$, then $\bar{\phi}_{x} \circ \bar{\psi}_{x}^{-1}$ is an isomorphism, and therefore both charts $(U, \phi)$ and $(V, \psi)$ induce the same vector space structure on $T_{x} M$. The set $T_{x} M$ endowed with such operations + and $\cdot$ is called the tangent space to $M$ at $x$ and we call its elements $[\gamma]$, with $\gamma \in \mathcal{C}^{p}(M, x)$, the tangent vectors to $M$ at $x$. 
An abstract-definable $\mathcal{C}^{p}$-map $f: M \rightarrow N$ induces at each point $x \in M$ a linear $\operatorname{map} d_{x} f: T_{x} M \rightarrow T_{f(x)} N$ given by

$$
d_{x} f([\gamma]):=[f \circ \gamma]
$$

Note that if $\gamma_{1} \sim_{c} \gamma_{2}$ and $(U, \phi)$ is a chart on $M$ at $x$ such that $\left(\phi \circ \gamma_{1}\right)^{\prime}(0)=$ $\left(\phi \circ \gamma_{2}\right)^{\prime}(0)$, then for any chart $(V, \psi)$ on $N$ at $f(x)$ we have $\psi \circ f \circ \gamma_{1}=(\psi \circ f \circ$ $\left.\phi^{-1}\right) \circ\left(\phi \circ \gamma_{2}\right)$ on a suitable domain. From the chain rule for definable maps, we obtain

$$
\left(\psi \circ f \circ \gamma_{1}\right)^{\prime}(0)=d_{\phi(x)}\left(\psi \circ f \circ \phi^{-1}\right)\left(\left(\phi \circ \gamma_{1}\right)^{\prime}(0)\right) .
$$

Hence, $\left(\psi \circ f \circ \gamma_{1}\right)^{\prime}(0)=\left(\psi \circ f \circ \gamma_{2}\right)^{\prime}(0)$. In other words, $f \circ \gamma_{1} \sim_{c} f \circ \gamma_{2}$. The linearity of $d_{x} f$ is attained as follows. If $(U, \phi)$ and $(V, \psi)$ are charts on $M$ and $N$, respectively, with $x \in U$ and $f(x) \in V$, then by the chain rule we get

$$
\bar{\psi}_{f(x)} \circ d_{x} f=d_{\phi(x)}\left(\psi \circ f \circ \phi^{-1}\right) \circ \bar{\phi}_{x}
$$

Since the right-hand side of the above equality is linear and $\bar{\psi}_{x}$ is a one-to-one linear map, the map $d_{x} f$ is linear as well. The above discussion can be summarized by saying that $d_{x} f$ is well-defined. We call $d_{x} f$ the differential of $f$ at $x$.

An immediate consequence of the definition of differential of abstract-definable $\mathcal{C}^{p}$ maps is the following.

Proposition 4.2 (Chain rule for abstract-definable maps). Let $f: M \rightarrow N$ and $g: N \rightarrow P$ be abstract-definable $\mathcal{C}^{p}$ maps between abstract-definable $\mathcal{C}^{p}$-manifolds. Then $g \circ f: M \rightarrow P$ is abstract-definable $\mathcal{C}^{p}$, and for any point $x$ in $M$ we have

$$
d_{x}(g \circ f)=d_{f(x)} g \circ d_{x} f
$$

Proposition 4.3. Let $U$ be an abstract-definable open subset of $M$. For each $x \in U$, the differential of the inclusion map $\iota: U \rightarrow M$ at $x$ is an isomorphism from $T_{x} U$ onto $T_{x} M$.

Proof. It suffices to prove that $d_{x} \iota$ is bijective. The injectivity of $d_{x} \iota$ follows immediately from the definition of differential at a point. Now, fix an abstractdefinable $\mathcal{C}^{p}$ curve $\gamma \in \mathcal{C}^{p}(M, x)$. We aim to find an $\alpha \in \mathcal{C}^{p}(U, x)$ such that $\iota \circ \alpha \sim_{c} \gamma$. Indeed, take $I \subseteq \operatorname{dom}(\gamma) \cap \gamma^{-1}(U)$ to be an open interval containing 
0 and $\alpha: I \rightarrow U$ to be the restriction $\left.\gamma\right|_{I}$. Since the map $\alpha: I \rightarrow U$ is abstractdefinable $\mathcal{C}^{p}$ (by Proposition 2.9) and $\alpha(0)=x, \alpha \in \mathcal{C}^{p}(M, x)$. For any chart $(V, \psi)$ on $M$ at $x$,

$$
(\psi \circ \iota \circ \alpha)^{\prime}(0)=\left(\left.\psi \circ \gamma\right|_{I}\right)^{\prime}(0)=(\psi \circ \gamma)^{\prime}(0)
$$

which shows that $[\gamma]=d_{x} \iota(\alpha)$. In summary, $\alpha$ is onto.

In view of Proposition 4.3, we identify $T_{x} U$ with $T_{x} M$ and $d_{x} \iota$ with the identity $\operatorname{map}_{\operatorname{id}_{x} M}$, for any abstract-definable open subset $U \subseteq M$ and any $x \in U$.

Proposition 4.4. Let $U \subseteq M$ and $V \subseteq N$ be abstract-definable open subsets, and let $f: M \rightarrow N$ be an abstract-definable $\mathcal{C}^{p}$ map such that $f(U) \subseteq V$. Denote by $g: U \rightarrow V$ the restriction of $f$ to $V$. Then, for each $x \in U$,

$$
d_{x} f=d_{x} g
$$

Proof. Let us denote by $\imath: U \rightarrow M$ and $\jmath: V \rightarrow N$ the natural inclusion maps. Then, $\jmath \circ g=f \circ \imath$. Since $g$ is abstract-definable $\mathcal{C}^{p}$,

$$
d_{g(x)} \jmath \circ d_{x} g=d_{x} f \circ d_{x} \imath
$$

by the chain rule. By recalling that $d_{x} \iota=\mathrm{id}_{T_{x} M}$ and $d_{g(x)} J=\mathrm{id}_{T_{f(x)} N}$, the result then follows.

Remark 4.5. For any point $y$ in $R^{m}$, note that $T: T_{y} R^{m} \rightarrow R^{m}$ defined as $T([\gamma]):=$ $\gamma^{\prime}(0)$ is a linear isomorphism. Thus, given a chart $(U, \phi)$ at a point $x \in M$, from the identifications above it follows that $T \circ d_{x} \phi=\bar{\phi}_{x}$. So, identifying $T_{y} R^{m}$ with $R^{m}$ and $T$ with $\operatorname{id}_{R^{m}}$, we get $d_{x} \phi=\bar{\phi}_{x}$. We will indeed adopt such identifications.

Fix a point $x$ in $M$, and a chart $(U, \phi)=\left(U, x^{1}, \ldots, x^{m}\right)$, with $x^{i}$ the coordinate functions of $\phi$. Because $d_{\phi(x)}\left(\phi^{-1}\right): R^{m} \rightarrow T_{x} M$ is a linear isomorphism, the elements

$$
\left.\frac{\partial}{\partial x^{1}}\right|_{x}:=d_{\phi(x)}\left(\phi^{-1}\right)\left(e_{1}\right),\left.\ldots \frac{\partial}{\partial x^{m}}\right|_{x}:=d_{\phi(x)}\left(\phi^{-1}\right)\left(e_{m}\right),
$$

form a basis for $T_{x} M$, where $\left\{e_{i}\right\}_{i=1}^{m}$ of $R^{m}$ is the standard basis for $R^{m}$. Thus, a tangent vector $v \in T_{x} M$ can be uniquely written as

$$
v=\left.v^{1} \frac{\partial}{\partial x^{1}}\right|_{x}+\cdots+\left.v^{m} \frac{\partial}{\partial x^{m}}\right|_{x}
$$


with $v^{1}, \ldots, v^{m} \in R^{m}$. Define a right action of the ring $\mathcal{C}^{p}(M)$ of all abstractdefinable $\mathcal{C}^{p}$ functions $f: M \rightarrow R$ on $T_{x} M$ by setting up

$$
v f:=d_{x} f(v)
$$

We also denote $v f$ by $\frac{\partial f}{\partial v}(x)$, and call it the directional derivative of $f$ at $x$ in the $v$-direction. The particular case $\left.\frac{\partial}{\partial x^{i}}\right|_{x} f$, also denoted by $\frac{\partial f}{\partial x^{i}}(x)$, is called the $i$ th partial derivative of $f$ at $x$. The $i$ th partial derivative of $f$ at $x$ can be expressed in terms of the chart $(U, \phi)$,

$$
\left.\frac{\partial}{\partial x^{i}}\right|_{x} f=d_{x} f\left(\left.\frac{\partial}{\partial x^{i}}\right|_{x}\right)=d_{x} f\left(d_{\phi(x)}\left(\phi^{-1}\right)\left(e_{i}\right)\right)=d_{\phi(x)}\left(f \circ \phi^{-1}\right)\left(e_{i}\right)=\frac{\partial\left(f \circ \phi^{-1}\right)}{\partial r^{i}}(\phi(x)),
$$

that is,

$$
\left.\frac{\partial}{\partial x^{i}}\right|_{x} f=\frac{\partial\left(f \circ \phi^{-1}\right)}{\partial r^{i}}(\phi(x))
$$

where $r^{1}, \ldots, r^{m}$ are the standard coordinates on $R^{m}$. From the equation (4.4) and the fact that the partial derivatives of definable $\mathcal{C}^{p}$ functions defined on a definable open subset of $R^{m}$ are derivations ${ }^{1}$, it follows immediately that

$$
\begin{gathered}
\frac{\partial(f+g)}{\partial x^{i}}(x)=\frac{\partial f}{\partial x^{i}}(x)+\frac{\partial g}{\partial x^{i}}(x), \\
\frac{\partial(f g)}{\partial x^{i}}(x)=\frac{\partial f}{\partial x^{i}}(x) g(x)+f(x) \frac{\partial g}{\partial x^{i}}(x),
\end{gathered}
$$

and

$$
\frac{\partial(a f)}{\partial x^{i}}(x)=a \frac{\partial f}{\partial x^{i}}(x)
$$

for any $f, g \in \mathcal{C}^{p}(M)$ and any $a \in R$. The equation (4.4) also allows us to compute the coordinates $\left(v^{1}, \ldots, v^{m}\right)$, related to the basis $\left\{\left.\frac{\partial}{\partial x^{i}}\right|_{x}\right\}_{i}$, of a given tangent vector $v \in T_{x} M$ in terms of $v$, namely

$$
v^{j}=v x^{j}
$$

where $x^{j}=r^{j} \circ \phi$.

Let $f: M \rightarrow N$ be an abstract-definable $\mathcal{C}^{p}$ map, $x$ a point in $M$, and charts $(U, \phi)=\left(U, x^{1}, \ldots, x^{m}\right),(V, \psi)=\left(V, y^{1}, \ldots, y^{n}\right)$ on $M$ and $N$, respectively, with $x \in U$ and $f(x) \in V$. Since each $d_{x} f\left(\left.\frac{\partial}{\partial x^{i}}\right|_{x}\right)$ is a tangent vector in $T_{f(x)} N$, it is a linear combination of the elements $\left.\frac{\partial}{\partial y^{1}}\right|_{f(x)}, \ldots,\left.\frac{\partial}{\partial y^{n}}\right|_{f(x)}$. It is not hard to see,

\footnotetext{
${ }^{1} \mathrm{~A} \mathbb{K}$-linear map $D: A \rightarrow A$ from an algebra $A$ over a field $\mathbb{K}$ to itself is said to be a derivation if it satisfies the Leibniz's law: $D(a b)=D(a) b+a D(b)$, for any $a, b \in A$.
} 
with a help from the chain rule and (4.4), that the coefficients of such a linear combination are given by

$$
d_{x} f\left(\left.\frac{\partial}{\partial x^{i}}\right|_{x}\right) y^{j}=\frac{\partial\left(r^{j} \circ \psi \circ f \circ \phi^{-1}\right)}{\partial r^{i}}(\phi(x)), j=1, \ldots, n .
$$

In summary,

$$
d_{x} f\left(\left.\frac{\partial}{\partial x^{i}}\right|_{x}\right)=\left.\sum_{j=1}^{n} \frac{\partial\left(r^{j} \circ \psi \circ f \circ \phi^{-1}\right)}{\partial r^{i}}(\phi(x)) \frac{\partial}{\partial y^{j}}\right|_{f(x)}, i=1, \ldots, m
$$

The equation (4.7) provides us the Jacobian matrix of $f$ at $x$

$$
\left[\frac{\partial\left(r^{j} \circ \psi \circ f \circ \phi^{-1}\right)}{\partial r^{i}}(\phi(x))\right]_{j i} .
$$

The subsequent result ensures that the Jacobian matrix of $f$ at $x$ does not depend on the choice of the charts at $x$ and $f(x)$.

Proposition 4.6 (Change of coordinates). Let $x$ be a point in $M$, and let $(U, \phi)=$ $\left(U, x^{1}, \ldots, x^{m}\right)$ and $(V, \psi)=\left(V, y^{1}, \ldots, y^{m}\right)$ be charts on $M$ with $x \in U \cap V$. Denote by $\left(\widetilde{x}^{1}, \ldots, \widetilde{x}^{m}\right)$ the component functions of the definable $\mathcal{C}^{p}$ diffeomorphism $\psi \circ \phi^{-1}: \phi(U \cap V) \rightarrow \psi(U \cap V)$. Then,

$$
\left.\frac{\partial}{\partial x^{i}}\right|_{x}=\left.\sum_{j=1}^{m} \frac{\partial \widetilde{x}^{j}}{\partial r^{i}}(\phi(x)) \frac{\partial}{\partial y^{j}}\right|_{x}
$$

Proof. Since $\phi^{-1}=\psi^{-1} \circ\left(\psi \circ \phi^{-1}\right)$ on $\phi(U \cap V)$,

$$
d_{\phi(x)}\left(\phi^{-1}\right)=d_{\psi(x)}\left(\psi^{-1}\right) \circ d_{\phi(x)}\left(\psi \circ \phi^{-1}\right)
$$

Recall, from [5] (p. 108), that if $f=\left(f_{1}, \ldots, f_{n}\right): U \subseteq R^{m} \rightarrow R^{n}$ is a definable map that is differentiable at $x \in U$, then $d_{x} f\left(e_{i}\right)=\sum_{j=1}^{n} \frac{\partial f_{j}}{\partial r^{i}}(x) e_{j}$. Particularly,

$$
d_{\phi(x)}\left(\psi \circ \phi^{-1}\right)\left(e_{i}\right)=\sum_{j=1}^{m} \frac{\partial \widetilde{x}^{j}}{\partial r^{i}}(\phi(x)) e_{j}
$$


Therefore,

$$
\begin{aligned}
\left.\frac{\partial}{\partial x^{i}}\right|_{x}=d_{\phi(x)}\left(\phi^{-1}\right)\left(e_{i}\right) & =d_{\psi(x)}\left(\psi^{-1}\right)\left(\sum_{j=1}^{m} \frac{\partial \widetilde{x}^{j}}{\partial r^{i}}(\phi(x)) e_{j}\right) \\
& =\sum_{j=1}^{m} \frac{\partial \widetilde{x}^{j}}{\partial r^{i}}(\phi(x)) d_{\psi(x)}\left(\psi^{-1}\right)\left(e_{j}\right) \\
& =\left.\sum_{j=1}^{m} \frac{\partial \widetilde{x}^{j}}{\partial r^{i}}(\phi(x)) \frac{\partial}{\partial y^{j}}\right|_{x}
\end{aligned}
$$

The disjoint union of all tangent spaces

$$
\bigsqcup_{x \in M} T_{x} M=\bigcup_{x \in M}\{x\} \times T_{x} M
$$

is called the tangent bundle of $M$ and is denoted $T M$. The set $T M$ can be made into an abstract-definable $\mathcal{C}^{p}$-manifold as follows. Let $(U, \phi)$ be a chart on $M$, then we denote

$$
T U:=\bigsqcup_{x \in U} T_{x} U \cong \bigsqcup_{x \in U} T_{x} M
$$

Define $\widetilde{\phi}: T U \rightarrow \phi(U) \times R^{m}$ to be

$$
\widetilde{\phi}\left(x,\left.\sum_{i=1}^{m} a_{i} \frac{\partial}{\partial x^{i}}\right|_{x}\right):=\left(\phi(x), a_{1}, \ldots, a_{m}\right) .
$$

Such a function is bijective with inverse $\widetilde{\phi}^{-1}$ given by the rule

$$
\left(\phi(x), a_{1}, \ldots, a_{m}\right) \mapsto\left(x,\left.\sum_{i=1}^{m} a_{i} \frac{\partial}{\partial x^{i}}\right|_{x}\right) .
$$

Proposition 4.7. The pair $\left(T M,\left\{\widetilde{\phi}: T U \rightarrow \phi(U) \times R^{m}\right\}_{(U, \phi) \in \mathcal{A}}\right)$, with each $\widetilde{\phi}$ as defined above, is an abstract-definable $\mathcal{C}^{p}$-manifold of dimension $2 \mathrm{~m}$.

Proof. For each $(U, \phi),(V, \psi) \in \mathcal{A}$ we have

$$
\widetilde{\phi}(T U \cap T V)=\phi(U \cap V) \times R^{m}
$$


which shows that $\widetilde{\phi}(T U \cap T V)$ are definable and open in $R^{2 m}$. Furthermore, the transition maps $\widetilde{\psi} \circ \widetilde{\phi}^{-1}$ are given by

$$
\left(\psi \circ \phi^{-1} \circ \mathrm{pr}_{1}, \mathrm{pr}_{2}\right)
$$

restricted to $\phi(U \cap V) \times R^{m}$, where $\mathrm{pr}_{1}$ and $\mathrm{pr}_{2}$ denote the projections $R^{2 m} \rightarrow R^{m}$ onto the first and the last $m$ coordinates, respectively. Hence the $\widetilde{\psi} \circ \widetilde{\phi}^{-1}$ are definable $\mathcal{C}^{p}$.

Proposition 4.8. The projection $\pi: T M \rightarrow M$ defined as

$$
\left(x, X_{x}\right) \mapsto x
$$

for any $x \in M$ and $X_{x} \in T_{x} M$, is an abstract-definable $\mathcal{C}^{p}$ map.

Proof. Let $(U, \phi)$ and $(\widetilde{\psi}, T V)$ be arbitrary charts on $M$ and $T M$, respectively. Since, $\phi \circ \pi \circ \widetilde{\psi}^{-1}$ restricted on $\widetilde{\psi}\left(\pi^{-1}(U) \cap T V\right)$ is just the definable $\mathcal{C}^{p}$ map $\phi \circ \psi^{-1} \circ \mathrm{pr}_{1}$ restricted to $\psi(U \cap V) \times R^{m}$, where $\mathrm{pr}_{1}: R^{2 m} \rightarrow R^{m}$ denotes the projection from $R^{2 m}$ onto the first $m$ coordinates, it follows that $\pi$ is abstractdefinable $\mathcal{C}^{p}$.

\subsection{The cotangent bundle}

Let $x$ a point in $M$. The cotangent space of $M$ at $x$, denoted by $T_{x}^{*} M$, is the dual vector space of the tangent space $T_{x} M$. An element of the cotangent space $T_{x}^{*} M$ is called a covector at $x$. Thus, a covector at $x$ is a linear function $T_{x} M \rightarrow R$. The disjoint union of all cotangent spaces of $M$ is called the cotangent bundle of $M$, and is denoted by $T^{*} M$.

If $(U, \phi)$ is a chart on $M$, then according to the previous section for any $x \in U$

$$
\left\{\left.\frac{\partial}{\partial x^{1}}\right|_{x}, \ldots,\left.\frac{\partial}{\partial x^{m}}\right|_{x}\right\}
$$

forms a basis for $T_{x} M$. Denote by

$$
\left\{\left.d x^{1}\right|_{x}, \ldots,\left.d x^{m}\right|_{x}\right\}
$$


the corresponding dual basis, i.e., the set of linear functions $\left.d x^{i}\right|_{x}: T_{x} M \rightarrow R$ satisfying

$$
\left.d x^{i}\right|_{x}\left(\left.\frac{\partial}{\partial x^{j}}\right|_{x}\right)=\delta_{i j}
$$

where $\delta_{i j}$ is the Kronecker delta and $i, j=1, \ldots, m$. So, given $\omega_{x} \in T_{x}^{*} M$ there are unique $c_{1}\left(\omega_{x}\right), \ldots, c_{m}\left(\omega_{x}\right) \in R$ with

$$
\omega_{x}=\left.\sum_{i=1}^{m} c_{i}\left(\omega_{x}\right) d x^{i}\right|_{x}
$$

Observe that when evaluating the above map at each $\left.\frac{\partial}{\partial x^{j}}\right|_{x}, j \in\{1, \ldots, m\}$, we obtain

$$
c_{i}\left(\omega_{x}\right)=\omega_{x}\left(\left.\frac{\partial}{\partial x^{i}}\right|_{x}\right) .
$$

Proposition 4.9 (Change of coordinates). Let $x$ be a point in $M$, and let $(U, \phi)=$ $\left(U, x^{1}, \ldots, x^{m}\right)$ and $(V, \psi)=\left(V, y^{1}, \ldots, y^{m}\right)$ be charts on $M$ with $x \in U \cap V$. Denote by $\left(\widetilde{y}^{1}, \ldots, \widetilde{y}^{m}\right)$ the component functions of the definable $\mathcal{C}^{p}$ diffeomorphism $\phi \circ \psi^{-1}: \psi(U \cap V) \rightarrow \phi(U \cap V)$. Then,

$$
\left.d x^{i}\right|_{x}=\left.\sum_{j=1}^{m} \frac{\partial \widetilde{y}^{i}}{\partial r^{j}}(\psi(x)) d y^{j}\right|_{x}
$$

Proof. From (4.11) and (4.12), it follows that

$$
\left.d x^{i}\right|_{x}=\left.\left.\sum_{j=1}^{m} d x^{i}\right|_{x}\left(\left.\frac{\partial}{\partial y^{j}}\right|_{x}\right) d y^{j}\right|_{x}
$$

By Proposition 4.6,

$$
\begin{aligned}
\left.d x^{i}\right|_{x} & =\left.\sum_{j=1}^{m}\left(\left.\sum_{l=1}^{m} \frac{\partial \widetilde{y}^{l}}{\partial r^{j}}(\psi(x)) d x^{i}\right|_{x}\left(\left.\frac{\partial}{\partial x^{l}}\right|_{x}\right)\right) d y^{j}\right|_{x} \\
& =\left.\sum_{j=1}^{m} \frac{\partial \widetilde{y}^{i}}{\partial r^{j}}(\psi(x)) d y^{j}\right|_{x} .
\end{aligned}
$$

Any chart $(U, \phi)$ on $M$ gives rise to a bijection $\widehat{\phi}: T^{*} U \rightarrow \phi(U) \times R^{m}$ defined as

$$
\left(x,\left.\sum_{i=1}^{m} c_{i} d x^{i}\right|_{x}\right) \mapsto\left(\phi(x), c_{1}, \ldots, c_{m}\right),
$$


where

$$
T^{*} U:=\bigsqcup_{x \in U} T_{x}^{*} U=\bigsqcup_{x \in U} T_{x}^{*} M .
$$

Its inverse $\widehat{\phi}^{-1}: \phi(U) \times R^{m} \rightarrow T^{*} U$ is the following

$$
\left(\phi(x), c_{1}, \ldots, c_{m}\right) \mapsto\left(x,\left.\sum_{i=1}^{m} c_{i} d x^{i}\right|_{x}\right)
$$

Proposition 4.10. The pair $\left(T^{*} M,\left\{\widehat{\phi}: T^{*} U \rightarrow \phi(U) \times R^{m}\right\}_{(U, \phi) \in \mathcal{A}}\right)$, with each $\widehat{\phi}$ as defined above, is an abstract-definable $\mathcal{C}^{p}$-manifold of dimension $2 \mathrm{~m}$.

Proof. Similar to that of Proposition 4.7.

Proposition 4.11. The projection $\pi: T^{*} M \rightarrow M$ defined as

$$
\left(x, \omega_{x}\right) \mapsto x,
$$

for any $x \in M$ and $\omega_{x} \in T_{x}^{*} M$, is an abstract-definable $\mathcal{C}^{p}$ map.

Proof. Similar to that of Proposition 4.8. 


\section{Chapter 5}

\section{Abstract-definable vector bundles}

This chapter is an adaptation of Subsections 12.3, 12.4, and 12.5 in ([30], pp. 133139), concerning respectively vector bundles, smooth sections and smooth frames, to the abstract-definable context. It provides background material necessary for introducing later on this text the objects upon which our cohomology theory is built.

\subsection{Abstract-definable vector bundles}

Definition 5.1. Let $\pi: E \rightarrow M$ be an abstract-definable $\mathcal{C}^{p}$ map between abstractdefinable $\mathcal{C}^{p}$ manifolds satisfying the conditions:

(i) for every $x \in M$ the fiber $E_{x}:=\pi^{-1}(x)$ at $x$ has the structure of a $d$ dimensional $R$-vector space;

(ii) $M$ has a finite abstract-definable open cover $\left\{\Omega_{j}\right\}_{j \in J}$ and for each $j \in J$ there exists an abstract-definable $\mathcal{C}^{p}$ diffeomorphism $\varphi_{j}: \pi^{-1}\left(\Omega_{j}\right) \rightarrow \Omega_{j} \times R^{d}$ such that the diagram

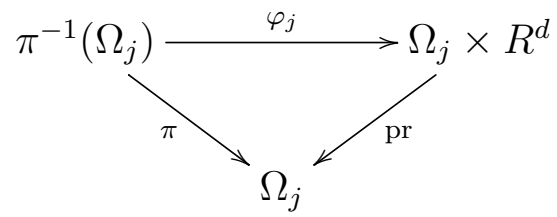

commutes, that is,

$$
\operatorname{pr} \circ \varphi_{j}=\pi \text { on } \pi^{-1}\left(\Omega_{j}\right),
$$


and for every $x \in \Omega_{j}$ the map $\left.\varphi_{j}\right|_{E_{x}}: E_{x} \rightarrow\{x\} \times R^{d}$ is a linear isomorphism, where pr: $\Omega_{j} \times R^{d} \rightarrow \Omega_{j}$ is the projection onto the first coordinates of the ordered pairs.

The triple $(E, M, \pi)$ is then said to be an abstract-definable $\mathcal{C}^{p}$ vector bundle of rank $d, E$ the total space, and $M$ the base space. Also, the collection $\left\{\left(\Omega_{j}, \varphi_{j}\right)\right\}_{j \in J}$ is called a local trivialization for $E$ and $\left\{\Omega_{j}\right\}_{j \in J}$ a trivializing open cover of $M$.

By abuse of notation, we will often designate an abstract-definable vector bundle $(E, M, \pi)$ as $\pi: E \rightarrow M, \pi$, or simply $E$.

In the sequel we give some examples of abstract-definable vector bundles of major importance for our purpose.

Example 5.1. Let $(M, \mathcal{A})$ be an abstract-definable $\mathcal{C}^{p}$-manifold of dimension $m$.

(1) The tangent bundle $T M$ of $M$ together with the projection $\pi: T M \rightarrow M$ as given in Theorem 4.8 is an abstract-definable $\mathcal{C}^{p}$ vector bundle whose fiber at $x \in M$ is the tangent space $\{x\} \times T_{x} M$ and

$$
\left\{\left(U,\left(\phi^{-1} \times \operatorname{id}_{R^{m}}\right) \circ \widetilde{\phi}\right):(U, \phi) \in \mathcal{A}\right\}
$$

where $\widetilde{\phi}$ is the induced chart on TM (see the discussion preceding Theorem 4.7), is a local trivialization.

(2) The cotangent bundle $T^{*} M$ of $M$ together with the projection $\pi: T^{*} M \rightarrow M$ as defined in Theorem 4.11 is an abstract-definable $\mathcal{C}^{p}$ vector bundle. The fiber at $x \in M$ is the cotangent space $\{x\} \times T_{x}^{*} M$ and

$$
\left\{\left(U,\left(\phi^{-1} \times \operatorname{id}_{R^{m}}\right) \circ \widehat{\phi}\right):(U, \phi) \in \mathcal{A}\right\}
$$

is a local trivialization, with $\widehat{\phi}$ the corresponding chart on $T M$ (see the discussion preceding Theorem 4.10).

(3) The triple $\left(M \times R^{d}, M, \pi\right)$, where $\pi: M \times R^{d} \rightarrow M$ denotes the projection onto $M$ is called the trivial vector bundle of rank $d$. The fiber at every $x \in M$ is just the vector space $\{x\} \times R^{d} \cong R^{d}$ and

$$
\left\{\left(U \times R^{d}, \operatorname{id}_{U \times R^{d}}\right):(U, \phi) \in \mathcal{A}\right\}
$$


is a local trivialization.

We could go on and define abstract-definable analogues of fiber preserving maps and bundle maps, as in [30] pp. 133 and 135 respectively. However, we sticked to the task of just introducing and developing in this chapter the least enough for what comes later.

\subsection{Sections and frames}

Definition 5.2. Let $\pi: E \rightarrow M$ be a an abstract-definable $\mathcal{C}^{p}$ vector bundle, and let $U$ be an abstract-definable open subset of $M$. A local section of $E$ over $U$ is an abstract-definable map $s: U \rightarrow E$ satisfying $\pi \circ s=\mathrm{id}_{U}$. If, in addition, $s$ is $\mathcal{C}^{p}$, then we say that $s$ is a $\mathcal{C}^{p}$ local section. In the case $U=M, s$ is called a $\left(\mathcal{C}^{p}\right)$ global section.

The condition $\pi \circ s=\mathrm{id}_{U}$ in the above definition means that $s$ maps each $x \in U$ into the fiber $E_{x}$. This allows us to sum sections and multiply them by abstractdefinable functions as it shows Lemma 5.3.

Example 5.2. Fix a chart $(U, \phi)=\left(U, x^{1}, \ldots, x^{m}\right)$ on the abstract-definable $\mathcal{C}^{p}$ manifold $M$ of dimension $m$.

(1) For each $i \in\{1, \ldots, m\}$ we define a map $\frac{\partial}{\partial x^{i}}: U \rightarrow T M$ by $x \mapsto\left(x,\left.\frac{\partial}{\partial x^{i}}\right|_{x}\right)$. We claim that $\frac{\partial}{\partial x^{i}}$ are $\mathcal{C}^{p}$ sections of $T M$ over $U$. Let $(T V, \widetilde{\psi})$ be any induced chart on $T M$ (see Section 1 of Chapter 4) such that $U \cap{\frac{\partial}{\partial x^{i}}}^{-1}(T V) \neq \emptyset$. The map $\widetilde{\psi} \circ \frac{\partial}{\partial x^{i}} \circ \phi^{-1}$ restricted to $\phi\left(U \cap{\frac{\partial}{\partial x^{i}}}^{-1}(T V)\right)=\phi(U \cap V)$ is then given by

$$
\phi(x) \mapsto\left(\left(\psi \circ \phi^{-1}\right)(\phi(x)), e_{i}\right),
$$

where $\left\{e_{1}, \ldots, e_{m}\right\}$ is the standard basis for $R^{m}$. Hence, such a restriction is definable $\mathcal{C}^{p}$.

(2) Similarly, the maps $d x^{i}: U \rightarrow T^{*} M$ defined by $\left.x \mapsto d x^{i}\right|_{x}$ with $i \in\{1, \ldots, m\}$ are $\mathcal{C}^{p}$ sections of the cotangent bundle $T^{*} M$ over $U$.

The next result ensures that the set of all $\mathcal{C}^{p}$ sections of an abstract-definable $\mathcal{C}^{p}$ vector bundle $\pi: E \rightarrow M$ over an abstract-definable open subset $U \subseteq M$ can 
be made into a module over the ring of all abstract-definable $\mathcal{C}^{p}$ functions on $U$. Strictly speaking, it is Proposition 12.9 ([30], p. 137) with the word "abstractdefinable" properly added, and their proofs are virtually the same.

Lemma 5.3. Let $s$ and $t$ be sections of an abstract-definable $\mathcal{C}^{p}$ vector bundle $\pi: E \rightarrow M$ over an abstract-definable open set $U \subseteq M$, and let $f: U \rightarrow R$ be an abstract-definable function. Then

(1) the sum $s+t$ defined by

$$
(s+t)(x):=s(x)+t(x) \in E_{x}, x \in M
$$

is a section of $E$ over $U$;

(2) the product $f s$ defined by

$$
(f s)(x):=f(x) \cdot s(x) \in E_{x}, x \in M
$$

is a section of $E$ over $U$.

If in addition $s, t$ and $f$ are $\mathcal{C}^{p}$, then $s+t$ and $f s$ are $\mathcal{C}^{p}$ sections.

Proof. Assume that $s$ and $t$ are $\mathcal{C}^{p}$ sections and $f$ is abstract-definable $\mathcal{C}^{p}$.

(1) We claim that $s+t$ is a $\mathcal{C}^{p}$ section. The proof of such an assertion follows from the two statements below.

The equality $\pi \circ(s+t)=\mathrm{id}_{U}$ holds. For any $x \in U$, we have $\pi \circ(s+t)(x)=$ $\pi(s(x)+t(x))$. Since $s(x)+t(x) \in E_{x}=\pi^{-1}(x), \pi(s(x)+t(x))=x$.

The map $s+t: U \rightarrow E$ is abstract-definable $\mathcal{C}^{p}$. Let $x \in U$ be an arbitrary point, $\Omega$ a trivializing open set for $E$ in which $x$ lies, and $\Omega^{\prime}:=\Omega \cap U$. In order to conclude the claim, it suffices to show that $s+t$ is abstract-definable $\mathcal{C}^{p}$ on $\Omega^{\prime}$ (see Example 2.7 and Proposition 2.10). Indeed, if $\varphi: \pi^{-1}(\Omega) \rightarrow \Omega \times R^{d}$ is the corresponding trivialization for $\Omega$, then the restriction $\left.\varphi\right|_{\pi^{-1}\left(\Omega^{\prime}\right)}: \pi^{-1}\left(\Omega^{\prime}\right) \rightarrow \Omega^{\prime} \times R^{d}$ is also a trivialization, which we denote by $\varphi^{\prime}$. In particular, we have $\left(\operatorname{pr}_{1} \circ \varphi^{\prime}\right)(s(z))=$ $\pi(s(z))=z$, for every $z \in \Omega^{\prime}$. This implies that

$$
\varphi^{\prime}(s(z))=(z, a(z))
$$


for some map $a: \Omega^{\prime} \rightarrow R^{d}$. Analogously,

$$
\varphi^{\prime}(t(z))=(z, b(z))
$$

where $b: \Omega^{\prime} \rightarrow R^{d}$ is a map. Since $\varphi^{\prime} \circ s$ and $\varphi^{\prime} \circ t$ are abstract-definable $\mathcal{C}^{p}$, so are $a$ and $b$. Furthermore, because $\varphi^{\prime}$ is linear on each fiber,

$$
\varphi^{\prime}((s+t)(z))=\varphi^{\prime}(s(z))+\varphi^{\prime}(t(z))=(z, a(z)+b(z)),
$$

for all $z \in \Omega^{\prime}$. Finally, the right-hand side of the above equality is abstractdefinable $\mathcal{C}^{p}$ as well as $\varphi^{\prime}$, hence it follows that $s+t$ is abstract-definable $\mathcal{C}^{p}$ on $\Omega^{\prime}$.

(2) The fact that $f s$ is a $\mathcal{C}^{p}$ section is shown in a similar way to that in (1).

Definition 5.4. A local frame for an abstract-definable $\mathcal{C}^{p}$ vector bundle $\pi: E \rightarrow$ $M$ of rank $d$ is a $d$-tuple of local sections $\left(s_{1}, \ldots, s_{d}\right)$ of $E$ over an abstract-definable open subset $U \subseteq M$ such that at each point $x \in U$, the elements $s_{1}(x), \ldots, s_{d}(x)$ form a basis for the fiber $E_{x}$. If in addition the sections $s_{1}, \ldots, s_{d}$ are $\mathcal{C}^{p}$, then $\left(s_{1}, \ldots, s_{d}\right)$ is called a $\mathcal{C}^{p}$ local frame for $E$ over $U$. In the case $U=M$, this $d$-tuple is said to be a $\left(\mathcal{C}^{p}\right)$ global frame.

Example 5.3. Fix a chart $(U, \phi)=\left(U, x^{1}, \ldots, x^{m}\right)$ on the abstract-definable $\mathcal{C}^{p}$ manifold $M$ of dimension $m$.

(1) In view of Example 5.2 and the fact that for each $x \in U$ the $m$-tuple $\left(\left.\frac{\partial}{\partial x^{1}}\right|_{x}, \ldots,\left.\frac{\partial}{\partial x^{m}}\right|_{x}\right)$ is a basis for the fiber $T_{x} M$ at $x$ of the tangent bundle $T M$ (see Section 1 of Chapter 4), it follows that $\left(\frac{\partial}{\partial x^{1}}, \ldots, \frac{\partial}{\partial x^{m}}\right)$ is a $\mathcal{C}^{p}$ frame for $T M$ over $U$.

(2) Similarly, by reason of Example 5.2 and Section 2 of Chapter 4, the m-tuple $\left(d x^{1}, \ldots, d x^{m}\right)$ forms a $\mathcal{C}^{p}$ frame for the cotangent bundle $T^{*} M$ over $U$, and we call it a coordinate frame.

Let us now describe a special local frame, which is the abstract-definable analogue of that depicted in Example 12.10 ([30], p. 138). Suppose $\pi: E \rightarrow M$ is an abstract-definable $\mathcal{C}^{p}$ vector bundle and the map $\varphi: \pi^{-1}(\Omega) \rightarrow \Omega \times R^{d}$ is a local trivialization of $E$. Then, there is a $\mathcal{C}^{p}$ local frame for $E$ over $\Omega$. Indeed, define 
maps $t_{1}, \ldots, t_{d}: \Omega \rightarrow E$ by

$$
t_{i}(x):=\left(\varphi^{-1} \circ \tilde{e}_{i}\right)(x)
$$

where $\tilde{e}_{i}: \Omega \rightarrow \Omega \times R^{d}$ is the abstract-definable $\mathcal{C}^{p}$ map given by the rule

$$
x \mapsto\left(x, e_{i}\right),
$$

with $\left(e_{1}, \ldots, e_{d}\right)$ the standard basis for $R^{d}$. Because $\varphi$ is an abstract-definable $\mathcal{C}^{p}$ diffeomorphism, each $t_{i}$ is abstract-definable $\mathcal{C}^{p}$. Also, from $\operatorname{pr} \circ \varphi=\pi$ where pr is the projection as in Definition 5.1, it follows that $\pi \circ t_{i}=\mathrm{id}_{\Omega}$. Lastly, for every $x \in \Omega$ the $d$-tuple $\left(t_{1}(x), \ldots, t_{d}(x)\right)$ is a basis for $E_{x}$, since $\left\{\tilde{e}_{1}(x), \ldots, \tilde{e}_{d}(x)\right\}$ is a basis for $\{x\} \times R^{d}$ and $\varphi^{-1}$ restricted to $\{x\} \times R^{d}$ is a linear isomorphism. We call $\left(t_{1}, \ldots, t_{d}\right)$ the $\mathcal{C}^{p}$ local frame associated with $\varphi$.

In view of this discussion, we may characterize the $\mathcal{C}^{p}$ sections over a trivialization of an abstract-definable $\mathcal{C}^{p}$ vector bundle in terms of the frame associated with such a trivialization. Again, this is done precisely as in Lemma 12.11 ([30], p. $138)$.

Lemma 5.5. Let $\varphi: \pi^{-1}(\Omega) \rightarrow \Omega \times R^{d}$ be a trivialization of an abstract-definable $\mathcal{C}^{p}$ vector bundle $\pi: E \rightarrow M$, and $\left(t_{1}, \ldots, t_{d}\right)$ the $\mathcal{C}^{p}$ local frame associated with $\varphi$. Then, a map $s:=\sum_{i=1}^{d} b^{i} t_{i}$, where $b^{i}$ are $R$-valued functions on $\Omega$, is a $\mathcal{C}^{p}$ section of $E$ over $\Omega$ if, and only if, its coefficients $b^{i}$ are abstract-definable $\mathcal{C}^{p}$.

Proof. Suppose $s=\sum_{i} b^{i} t_{i}$ is a $\mathcal{C}^{p}$ section of $E$ over $\Omega$. Note that for every $x$ in $\Omega$

$$
\varphi(s(x))=\sum_{i} b^{i}(x) \varphi\left(t_{i}(x)\right)=\sum_{i} b^{i}(x) \tilde{e}_{i}(x)=\left(x, b^{1}(x), \ldots, b^{d}(x)\right),
$$

that is,

$$
\varphi \circ s=\left(\operatorname{id}_{\Omega}, b^{1}, \ldots, b^{d}\right) .
$$

Since $\varphi \circ s$ is abstract-definable $\mathcal{C}^{p}$, all the functions $b^{i}$ are abstract-definable $\mathcal{C}^{p}$. Assume that $b^{1}, \ldots, b^{d}: \Omega \rightarrow R$ are abstract-definable $\mathcal{C}^{p}$ functions. From Lemma 5.3 , it follows immediately that $s$ is a $\mathcal{C}^{p}$ local section of $E$ over $\Omega$.

Proposition 5.6 below is an extension of Lemma 5.5 in the sense that in a similar fashion it characterizes $\mathcal{C}^{p}$ sections of an abstract-definable $\mathcal{C}^{p}$ vector bundle 
$\pi: E \rightarrow M$ over any abstract-definable open subset of $M$, unlike in Lemma 5.5 where the corresponding sets are elements of a trivializing open cover of $M$. Such a proposition plays fundamental role in allowing us to give a local description of the abstract-definable analogues of global smooth differential forms, examined in the next chapter. As in the whole chapter, both the statement and the proof of Proposition 5.6 are obtained from Proposition 12.12 ([30], p. 138) and its corresponding proof, through the addition of the word "abstract-definable" to its proper place.

Proposition 5.6. Let $\pi: E \rightarrow M$ be an abstract-definable $\mathcal{C}^{p}$ vector bundle of rank $d$, and $U$ an abstract-definable open subset of $M$. Suppose $\left(s_{1}, \ldots, s_{d}\right)$ is a $\mathcal{C}^{p}$ frame for $E$ over $U$. Then the map $s:=\sum_{i=1}^{d} c^{i} s_{i}$, where $c^{i}$ are $R$-valued functions on $U$, is a $\mathcal{C}^{p}$ section of $E$ over $U$ if and only if the coefficients $c^{i}$ are abstract-definable $\mathcal{C}^{p}$.

Proof. If the coefficients $c^{i}: U \rightarrow R$ are abstract-definable $\mathcal{C}^{p}$ functions, then from Lemma 5.3 it follows immediately that $s$ is a $\mathcal{C}^{p}$ local section of $E$ over $U$.

Conversely, assume that the section $s$ is $\mathcal{C}^{p}$. Let $x$ be an arbitrary point in $U, \Omega$ a trivializing open set for $(E, M, \pi)$ in which $x$ lies, $(\psi, W)$ chart on $M$ at $x$, and set $\Omega^{\prime}:=\Omega \cap U \cap W$. In order to prove that the functions $c^{i}$ are abstract-definable $\mathcal{C}^{p}$ on $U$, it suffices to show that such a condition is satisfied by $c^{i}$ on $\Omega^{\prime}$ (see Example 2.7 and Proposition 2.10). Indeed, if $\varphi: \pi^{-1}(\Omega) \rightarrow \Omega \times R^{d}$ is the corresponding trivialization for $\Omega$ then the restriction $\varphi^{\prime}:=\left.\varphi\right|_{\pi^{-1}\left(\Omega^{\prime}\right)}: \pi^{-1}\left(\Omega^{\prime}\right) \rightarrow \Omega^{\prime} \times R^{d}$ is also a trivialization. Let $\left(t_{1}, \ldots, t_{d}\right)$ be the $\mathcal{C}^{p}$ local frame associated with $\varphi^{\prime}$, and let $\sum_{i} b^{i} t_{i}$ and $\sum_{i} a_{j}^{i} t_{i}$ be the expressions of $s$ and $s_{j}$, respectively, in terms of the frame $\left(t_{1}, \ldots, t_{d}\right)$. By Lemma 5.5 , the functions $b^{i}, a_{j}^{i}: \Omega^{\prime} \rightarrow R$ are abstractdefinable $\mathcal{C}^{p}$. Putting the pieces together, we get

$$
\sum_{i} b^{i} t_{i}=s=\sum_{j} c^{j} s_{j}=\sum_{i, j} c^{j} a_{j}^{i} t_{i}
$$

Hence,

$$
b^{i}=\sum_{j} c^{j} a_{j}^{i}
$$

or in matrix notation

$$
\left[\begin{array}{c}
b^{1} \\
\vdots \\
b^{d}
\end{array}\right]=\left[\begin{array}{ccc}
a_{1}^{1} & \cdots & a_{d}^{1} \\
\vdots & \ddots & \vdots \\
a_{1}^{d} & \cdots & a_{d}^{d}
\end{array}\right]\left[\begin{array}{c}
c^{1} \\
\vdots \\
c^{d}
\end{array}\right] .
$$


Denote this square matrix by $A$. Since, at every $z \in \Omega^{\prime}$,

$$
\left[\begin{array}{ccc}
a_{1}^{1}(z) & \cdots & a_{d}^{1}(z) \\
\vdots & \ddots & \vdots \\
a_{1}^{d}(z) & \cdots & a_{d}^{d}(z)
\end{array}\right]
$$

is the transition matrix between the bases $\left\{s_{i}(z)\right\}_{i}$ and $\left\{t_{i}(z)\right\}_{i}, A$ is invertible. By Cramer's rule,

$$
c^{i}=\frac{\operatorname{det}\left(A_{i}\right)}{\operatorname{det}(A)}
$$

where $A_{i}$ is the matrix formed by replacing the $i$-th column of $A$ with the column vector on the left-hand side in the above matrix equation. Thus, each $c^{i}$ is an abstract-definable $\mathcal{C}^{p}$ function on $\Omega^{\prime}$. 


\section{Chapter 6}

\section{Abstract-definable forms}

In this chapter we introduce the elements for which our cohomology theory is established, the abstract-definable forms. They are defined in a global way, that is, as sections of specific abstract-definable vector bundles on an abstract-definable manifold. The first section deals with abstract-definable $\mathcal{C}^{p} 1$-forms. We begin by giving a characterization of them in terms of the coordinate frames, and in view of this we show that the pullback of an abstract-definable $\mathcal{C}^{p} 1$-form under an abstract-definable $\mathcal{C}^{p}$ map is an abstract-definable $\mathcal{C}^{p-1}$ 1-form. In Section 2 we generalize the results from the preceding section to abstract-definable $\mathcal{C}^{p} k$-forms. We give them a similar characterization to that of abstract-definable 1-forms, allowing us to conclude that the wedge product between an abstract-definable $\mathcal{C}^{p}$ $k$-form and an abstract-definable $\mathcal{C}^{q} l$-form is an abstract-definable $\mathcal{C}^{r}(k+l)$-form, where $r$ is the least of $p$ and $q$, and also that the pullback of an abstract-definable $\mathcal{C}^{p} k$-form via an abstract-definable $\mathcal{C}^{p}$ map is an abstract-definable $\mathcal{C}^{p} k$-form. All of these show that we cannot carry out the study of abstract-definable objects of finite classes of diferentiability towards a de Rham-like cohomology theory. The approach in this chapter is the same as in Sections 17 and 18 of Chapter 5 ([30], pp. 190-206), as we make it clear along the text.

Throughout the chapter, unless otherwise specified, $(M, \mathcal{A})$ and $(N, \mathcal{B})$ will denote arbitrary abstract-definable $\mathcal{C}^{p}$ manifolds of dimensions $m$ and $n$ respectively. 


\subsection{Abstract-definable 1-forms}

Definition 6.1. An abstract-definable $\left(\mathcal{C}^{p}\right)$ 1-form on $M$ is a $\left(\mathcal{C}^{p}\right)$ section $\omega$ of the cotangent bundle $\pi: T^{*} M \rightarrow M$.

In other words, an abstract-definable $\mathcal{C}^{p} 1$-form $\omega$ is an abstract-definable $\mathcal{C}^{p}$ map that assigns to every $x \in M$ a pair $\left(x, \omega_{x}\right)$, with $\omega_{x} \in T_{x}^{*} M$. As an abuse of notation, we shall often write the value of $\omega$ at a point $x$ in $M$ as simply $\omega_{x}$ instead of the pair $\left(x, \omega_{x}\right)$.

Recall from Example 5.3 that for a given chart $\left(U, x^{1}, \ldots, x^{m}\right)$ on $M$ the $m$-tuple $\left(d x^{1}, \ldots, d x^{m}\right)$ forms a $\mathcal{C}^{p}$ local frame for the cotangent bundle $T^{*} M$ over $U$. The following is then a special case of Propostion 5.6, where $T^{*} M$ and $d x^{i}$ play the roles of $E$ and $s_{i}$, respectively, in that proposition.

Lemma 6.2. Let $(U, \phi)=\left(U, x^{1}, \ldots, x^{m}\right)$ be a chart on $M$. The map $\omega:=$ $\sum_{i=1}^{m} \omega_{i} d x^{i}$, where $\omega_{i}$ are $R$-valued functions, is an abstract-definable $\mathcal{C}^{p}$ 1-form on $U$ if and only if the coefficients $\omega_{i}$ are abstract-definable $\mathcal{C}^{p}$.

The next result is inspired by the statement of Proposition 17.6 ([30], p. 194), and gives a characterization of the $\mathcal{C}^{p}$ abstract-definability of certain maps $M \rightarrow T^{*} M$ in terms of the coefficients of the 1-forms relative to the coordinate frames.

Theorem 6.3. Let $\omega: M \rightarrow T^{*} M$ be a map that satisfies the equality $\pi \circ \omega=\mathrm{id}_{M}$. The following are equivalent:

(1) $\omega$ is an abstract-definable $\mathcal{C}^{p}$ 1-form on $M$.

(2) For any chart $(U, \phi)$ on $M$, the restriction $\left.\omega\right|_{U}$ is given by

$$
\left.x \mapsto \sum_{i=1}^{m} \omega_{i}(x) d x^{i}\right|_{x}
$$

where the functions $\omega_{i}: U \rightarrow R$ are abstract-definable $\mathcal{C}^{p}$.

(3) For any point $x \in M$ there is a chart $(U, \phi)$ on $M$ at $x$ such that the restriction $\left.\omega\right|_{U}$ is given by

$$
\left.z \mapsto \sum_{i=1}^{m} \omega_{i}(z) d x^{i}\right|_{z}
$$

\footnotetext{
${ }^{1}$ Such a condition ensures that the value of $\omega$ at $x \in M$ lies in $T_{x}^{*} M$.
} 
where the functions $\omega_{i}: U \rightarrow R$ are abstract-definable $\mathcal{C}^{p}$.

(Recall that a chart $(U, \phi)$ is an open submanifold, see Example 2.7.)

Proof. $(1) \Rightarrow(2)$ Fix a chart $(U, \phi)=\left(U, x^{1}, \ldots, x^{m}\right)$ on $M$. Recall from Section 2 of Chapter 4 (on the cotangent bundle) that, for every $x \in U$, we can write

$$
\left(\left.\omega\right|_{U}\right)(x)=\left.\sum_{i=1}^{m} \omega_{i}(x) d x^{i}\right|_{x}
$$

where $\omega_{i}$ is a function on $U$. By (1), $\omega: M \rightarrow T^{*} M$ is an abstract-definable $\mathcal{C}^{p}$ 1-form, and hence the restriction $\left.\omega\right|_{U}$ is an abstract-definable $\mathcal{C}^{p}$ map (see Proposition 2.9). Moreover, $\left.\pi \circ \omega\right|_{U}=\mathrm{id}_{U}$. Therefore, $\left.\omega\right|_{U}$ is a $\mathcal{C}^{p}$ section of $T^{*} M$ over $U$. By Lemma 6.2, the functions $\omega_{i}$ are abstract-definable $\mathcal{C}^{p}$.

$(2) \Rightarrow(3)$ Immediate.

$(3) \Rightarrow(1)$ Let $x \in M$ be an arbitrary point, and by virtue of (3) let $(U, \phi)=$ $\left(U, x^{1}, \ldots, x^{m}\right)$ be a chart on $M$ at $x$ on which $\omega$ is written as $\sum_{i=1}^{m} \omega_{i} d x^{i}$, where $\omega_{i}$ is an abstract-definable $\mathcal{C}^{p}$ function on $U$. Consider the induced chart $\widehat{\phi}: T^{*} U \rightarrow$ $\phi(U) \times R^{m}$ on $T^{*} M$ (see Section 2 of Chapter 4). Clearly, $\omega(x) \in T^{*} U$. We come to the conclusion that $\omega$ is an abstract-definable $\mathcal{C}^{p} 1$-form on $M$ if we prove, on account of Proposition 3.10, that $\widehat{\phi} \circ \omega \circ \phi^{-1}$ restricted to $\phi\left(U \cap \omega^{-1}\left(T^{*} U\right)\right)$ is definable and is extended by a definable $\mathcal{C}^{p}$ map defined on a definable open subset of $R^{m}$.

Indeed, observe that the function $\left.\widehat{\phi} \circ \omega \circ \phi^{-1}\right|_{\phi\left(U \cap \omega^{-1}\left(T^{*} U\right)\right)}$ is given by

$$
\phi(z) \mapsto\left(\phi(z), \omega_{1}(z), \ldots, \omega_{m}(z)\right),
$$

or equivalently, by the map

$$
\left(\operatorname{id}_{\phi\left(U \cap \omega^{-1}\left(T^{*} U\right)\right)},\left.\omega_{1} \circ \phi^{-1}\right|_{\phi\left(U \cap \omega^{-1}\left(T^{*} U\right)\right)}, \ldots,\left.\omega_{m} \circ \phi^{-1}\right|_{\phi\left(U \cap \omega^{-1}\left(T^{*} U\right)\right)}\right)
$$

By Lemma 6.2, the map $\left.\omega\right|_{U}$ is in particular abstract-definable, and thereby $\omega^{-1}\left(T^{*} U\right)=\left(\left.\omega\right|_{U}\right)^{-1}\left(T^{*} U\right)$, since $\pi \circ \omega=\mathrm{id}_{M}$, is an abstract-definable subset of the abstract-definable $\mathcal{C}^{p}$ manifold $(U,\{\phi: U \rightarrow \phi(U)\})$. Hence, $\phi\left(U \cap \omega^{-1}\left(T^{*} U\right)\right)$ is definable. Because the maps $\omega_{i}$ are abstract-definable, the restriction of each $\omega_{i} \circ \phi^{-1}$ to $\phi\left(U \cap \omega^{-1}\left(T^{*} U\right)\right)$ is definable. From (6.1), it follows then that $\hat{\phi} \circ \omega \circ$ $\left.\phi^{-1}\right|_{\phi\left(U \cap \omega^{-1}\left(T^{*} U\right)\right)}$ is also definable. 
Finally, note that in view of $(3)$

$$
\left(\operatorname{id}_{\phi(U)}, \omega_{1} \circ \phi^{-1}, \ldots, \omega_{m} \circ \phi^{-1}\right)
$$

is a definable $\mathcal{C}^{p}$ map defined on the definable open set $\phi(U) \subseteq R^{m}$ extending the map in (6.1). This finishes the proof.

We may now give an example of an abstract-definable $\mathcal{C}^{p}$ 1-form with $p>0$ in which we have great interest.

Example 6.1 (Differential of an abstract-definable function). Consider an abstractdefinable $\mathcal{C}^{p}$ function $f: M \rightarrow R$ with $p \geq 2$. We then have an induced map df $: M \rightarrow T^{*} M$ given by

$$
x \mapsto\left(x, d_{x} f\right)
$$

where $d_{x} f: T_{x} M \rightarrow R$ denotes the differential of $f$ at $x$ (see Section 1 of Chapter 4 on the tangent bundle). Let $(U, \phi)=\left(U, x^{1}, \ldots, x^{m}\right)$ be an arbitrary chart on $M$. We know from Section 2 of Chapter 4 that for any $x \in U$

$$
d_{x} f=\left.\sum_{i=1}^{m} c_{i}(x) d x^{i}\right|_{x}
$$

with $c_{i}(x) \in R$. Evaluating $d_{x} f$ at each vector $\left.\frac{\partial}{\partial x^{i}}\right|_{x}$ and recalling that

$$
\frac{\partial f}{\partial x^{i}}(x):=\left.\frac{\partial}{\partial x^{i}}\right|_{x} f=d_{x} f\left(\frac{\partial}{\partial x^{i}}\right)=\frac{\partial\left(f \circ \phi^{-1}\right)}{\partial r^{i}}(\phi(x)),
$$

(see (4.4) in Section 1 of Chapter 4) we conclude

$$
c_{i}(x)=\frac{\partial\left(f \circ \phi^{-1}\right)}{\partial r^{i}}(\phi(x)) .
$$

In other words, $c_{i}$ is the map $\frac{\partial f}{\partial x^{i}}: U \rightarrow R$, which is defined as

$$
x \mapsto \frac{\partial\left(f \circ \phi^{-1}\right)}{\partial r^{i}}(\phi(x)) .
$$

We emphasize that $U$ is viewed as an abstract-definable $\mathcal{C}^{p}$ manifold with atlas $\{\phi\}$. From the fact that $f$ is abstract-definable $\mathcal{C}^{p}$, it follows that $f \circ \phi^{-1}: \phi(U) \rightarrow R$ is a definable $\mathcal{C}^{p}$ function, and consequently

$$
\frac{\partial\left(f \circ \phi^{-1}\right)}{\partial r^{i}}=\frac{\partial f}{\partial x^{i}} \circ \phi^{-1}
$$


is definable $\mathcal{C}^{p-1}$. In summary, the function $\frac{\partial f}{\partial x^{i}}$ is abstract-definable $\mathcal{C}^{p-1}$. Hence, on account of Theorem 6.3 the differential df of $f$ is an abstract-definable $\mathcal{C}^{p-1}$ 1-form on $M$. We finish this example by pointing out, from the above discussion, that in every chart $\left(U, x^{1}, \ldots, x^{m}\right)$ on $M$ the differential df of $f$ has the following local expression

$$
d f=\sum_{i=1}^{m} \frac{\partial f}{\partial x^{i}} d x^{i} .
$$

Let $F: N \rightarrow M$ be an abstract-definable $\mathcal{C}^{p}$ map. For any abstract-definable $\mathcal{C}^{p}$ function $g: M \rightarrow R$, we define the pullback of $g$ by $F$ to be the composition

$$
F^{*} g:=g \circ F
$$

which is clearly an abstract-definable $\mathcal{C}^{p}$ function on $N$. Now, let $\omega$ be a map

$$
y \mapsto\left(y, \omega_{y}\right): M \rightarrow T^{*} M
$$

The pullback of $\omega$ by $F$ is the map $F^{*} \omega: N \rightarrow T^{*} N$ given by

$$
x \mapsto\left(x,\left(F^{*} \omega\right)_{x}\right),
$$

where $\left(F^{*} \omega\right)_{x}: T_{x} N \rightarrow R$ is the following linear function

$$
v \mapsto \omega_{F(x)}\left(d_{x} F(v)\right)
$$

Remark 6.4. We draw the reader's attention to the point that the set of all maps $\omega$ from $M$ to $T^{*} M$ such that $\pi \circ \omega=\mathrm{id}_{M}$, where $\pi: T^{*} M \rightarrow M$ is the projection $\left(x, \omega_{x}\right) \mapsto x$, together with the operations

$$
(\omega+\tau)_{x}:=\omega_{x}+\tau_{x},(r \omega)_{x}:=r \omega_{x}, \text { and }(f \omega)_{x}:=f(x) \omega_{x},
$$

for $r \in R, f \in \mathcal{F}(M)$ (the ring of all $R$-valued functions on $M$ ) and $x \in M$, forms an $R$-vector space and a $\mathcal{F}(M)$-module.

We end this section with the verification that the pullback $F^{*} \omega$ of an abstractdefinable 1-form $\omega$ under an abstract-definable $\mathcal{C}^{p}$ map $F: N \rightarrow M$ is an abstractdefinable 1-form on $N$ (of a smaller differentiability class, though). As a preparation for this, we first prove that pullback commutes with differential, and is distributive with respect to the sum and scalar multiplication. 
Lemma 6.5 below is an abstract-definable version of Propositions 17.10 and 17.11 ([30], pp. 196 and 197). The proof here is virtually identical to those of these propositions.

Lemma 6.5. Let $F: N \rightarrow M$ be an abstract-definable $\mathcal{C}^{p}$ map, $g: M \rightarrow R$ an abstract-definable $\mathcal{C}^{p}$ function, and let $\omega, \tau: M \rightarrow T^{*} M$ be maps satisfying $\pi \circ \omega=$ $i d_{M}=\pi \circ \tau$. Then,

(1) $F^{*}(d g)=d\left(F^{*} g\right)$, where $d g, d\left(F^{*} g\right)$ are the differentials of $g$ and $F^{*} g$, respectively (see Example 6.1);

(2) $F^{*}(\omega+\tau)=F^{*} \omega+F^{*} \tau$;

(3) $F^{*}(g \omega)=\left(F^{*} g\right)\left(F^{*} \omega\right)$.

Proof. Let $x \in N$ be an arbitrary point and let $v \in T_{x} N$ be any vector.

By the chain rule,

$$
\left(F^{*} d g\right)_{x}(v)=(d g)_{F(x)}\left(d_{x} F(v)\right)=d_{F(x)} g\left(d_{x} F(v)\right)=d_{x}(g \circ F)(v)=d_{x}\left(F^{*} g\right)(v),
$$

hence (1) follows. From Remark 6.4, we obtain

$$
\begin{aligned}
\left(F^{*}(\omega+\tau)\right)_{x}(v)=(\omega+\tau)_{F(x)}\left(d_{x} F(v)\right) & =\omega_{F(x)}\left(d_{x} F(v)\right)+\tau_{F(x)}\left(d_{x} F(v)\right) \\
& =\left(F^{*} \omega\right)_{x}(v)+\left(F^{*} \tau\right)_{x}(v)
\end{aligned}
$$

and

$$
\begin{aligned}
\left(F^{*}(g \omega)\right)_{x}(v)=(g \omega)_{F(x)}\left(d_{x} F(v)\right) & =g(F(x)) \omega_{F(x)}\left(d_{x} F(v)\right) \\
& =(g \circ F)(x)\left(F^{*} \omega\right)_{x}(v) \\
& =\left(\left(F^{*} g\right)\left(F^{*} \omega\right)\right)_{x}(v),
\end{aligned}
$$

completing then the proof of (2) and (3).

Now we are ready to establish the abstract-definable version of Proposition 17.12 ([30], p. 197). Again, the proof of Proposition 17.12 works perfectly in our setting, only remaining to look out the definability of the objects involved. 
Proposition 6.6. The pullback $F^{*} \omega$ of an abstract-definable $\mathcal{C}^{p} 1$-form $\omega$ on $M$ under an abstract-definable $\mathcal{C}^{p}$ map $F: N \rightarrow M$ with $p \geq 2$ is an abstract-definable $\mathcal{C}^{p-1} 1$-form on $N$.

Proof. In order to conclude that $F^{*} \omega$ is an abstract-definable $\mathcal{C}^{p-1} 1$-form on $N$ it suffices by Theorem 6.3 to check that for every $x \in N$ there is a chart $(V, \psi)$ on $N$ at $x$ in which $F^{*} \omega$ is given by $\sum_{i=1}^{n} \tau_{i} d x^{i}$, with each $\tau_{i}: V \rightarrow R$ an abstract-definable $\mathcal{C}^{p-1}$ function. Indeed, fix $x \in N$ and let $(U, \phi)=\left(U, x^{1}, \ldots, x^{m}\right)$ be a chart on $M$ at $F(x)$. Since $F$ is continuous, there is an abstract-definable open set $\Omega \subseteq N$ of $x$ with $F(\Omega) \subseteq U$ (see Proposition 2.15). From Example 2.7, it follows that $\mathcal{B} \cup \mathcal{B}(\Omega)$ is an abstract-definable $\mathcal{C}^{p}$ atlas on $N$ which is $\sim$-equivalent to $\mathcal{B}$, where $\mathcal{B}(\Omega)$ denotes the abstract-definable $\mathcal{C}^{p}$ atlas $\left\{\left(\Omega \cap W,\left.\varphi\right|_{\Omega \cap W}\right):(W, \varphi) \in \mathcal{B}\right\}$ on $\Omega$. Particularly, there is a chart $(V, \psi)$ in $\mathcal{B} \cup \mathcal{B}(\Omega)$ satisfying $F(V) \subseteq U$. Observe that, for the sake of Theorem 6.3, the map $\omega$ is written on $U$ as $\omega=\sum_{i=1}^{m} \omega_{i} d x^{i}$ with $\omega_{i}: U \rightarrow R$ abstract-definable $\mathcal{C}^{p}$. Consequently, for each $z \in V$ and $v \in T_{z} N$

$$
\begin{aligned}
\left(F^{*} \omega\right)_{z}(v) & =\omega_{F(z)}\left(d_{z} F v\right)=\left(\left.\sum_{i=1}^{m} \omega_{i}(F(z)) d x^{i}\right|_{F(z)}\right)\left(d_{z} F v\right) \\
& =\left.\sum_{i=1}^{m}\left(\omega_{i} \circ F\right)(z) d x^{i}\right|_{F(z)}\left(d_{z} F v\right) \\
& =\sum_{i=1}^{m}\left(\omega_{i} \circ F\right)(z)\left(F^{*} d x^{i}\right)_{z}(v) .
\end{aligned}
$$

(The last equality follows from the chain rule and Lemma 6.5.) In other words,

$$
\left.F^{*} \omega\right|_{V}=\left.\left.\sum_{i=1}^{m} F\right|_{V} ^{*} \omega_{i} F\right|_{V} ^{*} d x^{i} .
$$

Applying Lemma 6.5 and (6.2) from Example 6.1, we obtain respectively the first and second equalities below

$$
\begin{aligned}
\left.F^{*} \omega\right|_{V} & =\sum_{i=1}^{m}\left(\left.\omega_{i} \circ F\right|_{V}\right) d\left(\left.x^{i} \circ F\right|_{V}\right) \\
& =\sum_{i=1}^{m}\left(\left.\omega_{i} \circ F\right|_{V}\right) \sum_{j=1}^{n} \frac{\partial\left(\left.x^{i} \circ F\right|_{V}\right)}{\partial y^{j}} d y^{j} \\
& =\sum_{i=1}^{m} \sum_{j=1}^{n}\left(\left.\omega_{i} \circ F\right|_{V}\right) \frac{\partial\left(\left.x^{i} \circ F\right|_{V}\right)}{\partial y^{j}} d y^{j} .
\end{aligned}
$$


Since the coefficients

$$
\left(\left.\omega_{i} \circ F\right|_{V}\right) \frac{\partial\left(\left.x^{i} \circ F\right|_{V}\right)}{\partial y^{j}}
$$

are abstract-definable $\mathcal{C}^{p-1} R$-valued functions on $V$, the proof is done.

\subsection{Abstract-definable $k$-forms}

We now generalize the construction of abstract-definable $\mathcal{C}^{p}$ 1-forms on $M$ to abstract-definable $\mathcal{C}^{p} k$-forms. In summary, we will apply the notions of multilinear algebra in Appendix A to the cotangent space $T_{x}^{*} M$ of $M$ at a point $x \in M$, as in the standard setting.

Notation. For the remainder of the chapter, I denotes a $k$-tuple $\left(i_{1}, \ldots, i_{k}\right)$, where $1 \leq i_{1}<\cdots<i_{k} \leq m, d x^{I}$ is a short for $d x^{i_{1}} \wedge \cdots \wedge d x^{i_{k}},\left.d x^{I}\right|_{x}$ designates $\left.\left.d x^{i_{1}}\right|_{x} \wedge \cdots \wedge d x^{i_{k}}\right|_{x}$, and similarly, for the increasing multi-index $J:=\left(j_{1}, \ldots, j_{l}\right)$, we denote $d x^{J}:=d x^{j_{1}} \wedge \cdots \wedge d x^{j_{l}}$ and $\left.d x^{J}\right|_{x}:=\left.\left.d x^{j_{1}}\right|_{x} \wedge \cdots \wedge d x^{j_{l}}\right|_{x}$.

Mimicking the construction of the tangent and cotangent bundles we form the set

$$
\bigwedge^{k} T^{*} M:=\bigsqcup_{x \in M} \bigwedge^{k} T_{x}^{*} M=\bigcup_{x \in M}\{x\} \times \bigwedge^{k} T_{x}^{*} M
$$

of all alternating $k$-tensors at all points of $M$. We call such a set the $k$ th exterior power of the cotangent bundle.

Let $(U, \phi)$ be a chart on $M$. There is a bijection $\bar{\phi}: \bigwedge^{k} T^{*} U \rightarrow \phi(U) \times R^{\left(\begin{array}{c}m \\ k\end{array}\right)}$ given by

$$
\left(x, \omega_{x}\right) \mapsto\left(\phi(x),\left(a_{I}\left(\omega_{x}\right)\right)_{I}\right),
$$

where $\omega_{x}=\left.\sum_{I} a_{I}\left(\omega_{x}\right) d x^{I}\right|_{x}$ on $U$, and

$$
\bigwedge^{k} T^{*} U:=\bigcup_{x \in U}\{x\} \times \bigwedge^{k} T_{x}^{*} U
$$

Thus, as in the cases of tangent and cotangent bundles, we have the following.

Proposition 6.7. The pair

$$
\left(\bigwedge^{k} T^{*} M,\left\{\bar{\phi}: \bigwedge^{k} T^{*} U \rightarrow \phi(U) \times R^{\left(\begin{array}{c}
m \\
k
\end{array}\right)}:(U, \phi) \in \mathcal{A}\right\}\right)
$$


with each $\bar{\phi}$ as defined in (6.7), is an abstract-definable $\mathcal{C}^{p}$ manifold of dimension $m+\left(\begin{array}{c}m \\ k\end{array}\right)$.

Proof. Similar to that of Proposition 4.7.

Proposition 6.8. The projection $\pi: \bigwedge^{k} T^{*} M \rightarrow M$ given by

$$
\left(x, \omega_{x}\right) \mapsto x,
$$

for any $x \in M$ and $\omega_{x} \in \bigwedge^{k} T_{x}^{*} M$, is an abstract-definable $\mathcal{C}^{p}$ map.

Proof. Similar to that of Proposition 4.8.

By virtue of Propositions 6.7 and 6.8, $\pi: \bigwedge^{k} T^{*} M \rightarrow M$ is an abstract-definable $\mathcal{C}^{p}$ vector bundle of rank $\left(\begin{array}{c}m \\ k\end{array}\right)$ whose fibers $\pi^{-1}(x)$ are the vector spaces $\bigwedge^{k} T_{x}^{*} M$.

At this point we may introduce the notion of abstract-definable $\mathcal{C}^{k} k$-forms.

Definition 6.9. An abstract-definable $\left(\mathcal{C}^{p}\right) k$-form on $M$ is a $\left(\mathcal{C}^{p}\right)$ section $\omega$ of the $k$ th exterior power of the cotangent bundle $\pi: \bigwedge^{k} T^{*} M \rightarrow M$.

Since $\bigwedge^{0} T^{*} M \cong M \times R$, the vector space of all abstract-definable $\mathcal{C}^{p}$ 0-forms identifies with that of all abstract-definable $\mathcal{C}^{p}$ functions on $M$.

Remark 6.10. For a fixed chart $(U, \phi)=\left(U, x^{1}, \ldots, x^{m}\right)$ on $M$ and for any $x \in U$, the set

$$
\left\{\left.d x^{I}\right|_{x}\right\}_{I}
$$

forms a basis for the $R$-vector space $\bigwedge^{k} T_{x}^{*} M$. The order on such a basis is the lexicographic order over the increasing multi-indices $I$ of length $k$. Observe that for the case $k=1$ the set in (6.8), viewed as an ordered basis, reduces to the ordered basis $\left\{\left.d x^{1}\right|_{x}, \ldots,\left.d x^{m}\right|_{x}\right\}$ of $\bigwedge^{1} T_{x}^{*} M=T_{x}^{*} M$.

Lemma 6.11. Given a chart $(U, \phi)=\left(U, x^{1}, \ldots, x^{m}\right)$ on $M$, the $\left(\begin{array}{c}m \\ k\end{array}\right)$-tuple $\left(d x^{I}\right)_{I}$ forms a $\mathcal{C}^{p}$ local frame for the abstract-definable $\mathcal{C}^{p}$ vector bundle $\bigwedge^{k} T^{*} M$ over $U$.

Proof. We claim that for each $I$ the map $d x^{I}: U \rightarrow \bigwedge^{k} T^{*} M$ given by

$$
\left.x \mapsto d x^{I}\right|_{x}
$$


is a $\mathcal{C}^{p}$ local section of $\bigwedge^{k} T^{*} M$ over $U$. Indeed, for any induced chart $\left(\bigwedge^{k} T^{*} V, \bar{\psi}\right)$ on $\bigwedge^{k} T^{*} M$ (see (6.7) in this section) with $\emptyset \neq\left(d x^{I}\right)^{-1}\left(\bigwedge^{k} T^{*} V\right) \subseteq U$, the set $\phi\left(\left(d x^{I}\right)^{-1}\left(\bigwedge^{k} T^{*} V\right)\right)$ is definable, since it equals $\phi(U \cap V)$, and $\bar{\psi} \circ d x^{I} \circ \phi^{-1}$ restricted to $\phi\left(\left(d x^{I}\right)^{-1}\left(\bigwedge^{k} T^{*} V\right)\right.$ is a $\mathcal{C}^{p}$-map, because is given by

$$
\phi(x) \mapsto\left(\left(\psi \circ \phi^{-1}\right)(\phi(x)), e_{j}\right)
$$

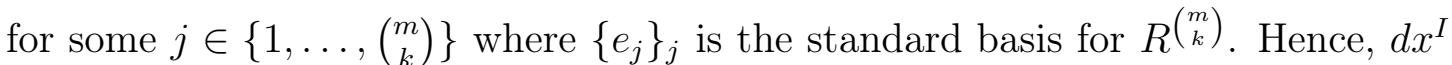
is a $\mathcal{C}^{p}$ local section of $\bigwedge^{k} T^{*} M$. From Remark 6.10, it follows that for each $x$ in $U$ the sequence $\left(\left.d x^{I}\right|_{x}\right)_{I}$ is a basis for the fiber $\bigwedge^{k} T_{x}^{*} M$. Therefore, $\left(d x^{I}\right)_{I}$ is a $\mathcal{C}^{p}$ local frame for $\wedge^{k} T^{*} M$.

Lemma 6.12. Let $(U, \phi)=\left(U, x^{1}, \ldots, x^{m}\right)$ be a chart on $M$. The map $\omega:=$ $\sum_{I} \omega_{I} d x^{I}$, where $\omega_{I}$ are $R$-valued functions on $U$, is an abstract-definable $\mathcal{C}^{p} k$ form on $U$ if and only if the coefficients $\omega_{I}$ are abstract-definable $\mathcal{C}^{p}$.

Proof. Recall from Lemma 6.11 that $\left(d x^{I}\right)_{I}$ is a $\mathcal{C}^{p}$ local frame for $\bigwedge^{k} T^{*} M$ over $U$. By applying Proposition 5.6 to the abstract-definable $\mathcal{C}^{p}$ vector bundle $\bigwedge^{k} T^{*} M$ and to the $\mathcal{C}^{p}$ local frame $\left(d x^{I}\right)_{I}$, we immediately obtain the result.

In view of Lemmas 6.11 and 6.12 above we give a characterization of abstractdefinable $\mathcal{C}^{p} k$-forms similar to that of abstract-definable $\mathcal{C}^{p} 1$-forms in Theorem 6.3 .

Theorem 6.13. Let $\omega: M \rightarrow \bigwedge^{k} T^{*} M$ that satisfies the equality $\pi \circ \omega=\operatorname{id}_{M}$. The following are equivalent.

(1) $\omega$ is an abstract-definable $\mathcal{C}^{p} k$-form on $M$.

(2) For any chart $(U, \phi)=\left(U, x^{1}, \ldots, x^{m}\right)$ on $M$, the restriction $\left.\omega\right|_{U}$ is given by

$$
\left.x \mapsto \sum_{I} \omega_{I}(x) d x^{I}\right|_{x}
$$

where the functions $\omega_{I}: U \rightarrow R$ are abstract-definable $\mathcal{C}^{p}$.

(3) For any point $x \in M$ there is a chart $(U, \phi)=\left(U, x^{1}, \ldots, x^{m}\right)$ on $M$ at $x$ such that the restriction $\left.\omega\right|_{U}$ is given by

$$
\left.z \mapsto \sum_{I} \omega_{I}(z) d x^{I}\right|_{z}
$$


where the functions $\omega_{I}: U \rightarrow R$ are abstract-definable $\mathcal{C}^{p}$.

(Recall that a chart $(U, \phi)$ is an open submanifold, see Example 2.7.)

Proof. Similar to that of Proposition 6.3.

Let $\omega, \tau$ be maps $x \mapsto\left(x, \omega_{x}\right): M \rightarrow \bigwedge^{k} T^{*} M$ and $x \mapsto\left(x, \tau_{x}\right): M \rightarrow \bigwedge^{l} T^{*} M$, respectively. The wedge product of $\omega$ and $\tau$ is the map $\omega \wedge \tau: M \rightarrow \wedge^{k+l} T^{*} M$ given by

$$
x \mapsto\left(x, \omega_{x} \wedge \tau_{x}\right) .
$$

In the case the maps $\omega$ and $\tau$ are abstract-definable forms, then so is their wedge product, as it shows Proposition 6.14. Such a proposition is obtained from Proposition 18.10 ([30], p. 206) by adding the word "abstract-definable".

Proposition 6.14. If $\omega$ is an abstract-definable $\mathcal{C}^{p} k$-form on $M$ and $\tau$ is an abstract-definable $\mathcal{C}^{q} l$-form on $M$, then $\omega \wedge \tau$ is an abstract-definable $\mathcal{C}^{r}(k+l)$ form on $M$ with $r:=\min \{p, q\}$.

Proof. Let $\omega, \tau$ be abstract-definable $\mathcal{C}^{p} k$ - and $\mathcal{C}^{q} l$-forms on $M$ respectively, and fix a chart $(U, \phi)=\left(U, x^{1}, \ldots, x^{m}\right)$ on $M$. By Theorem 6.13, we have

$$
\left.\omega\right|_{U}=\sum_{I} \omega_{I} d x^{I} \text { and }\left.\tau\right|_{U}=\sum_{J} \tau_{J} d x^{J},
$$

for abstract-definable $\mathcal{C}^{p}$ and $\mathcal{C}^{q}$ functions $\omega_{I}, \tau_{J}: U \rightarrow R$ respectively. The computation of the wedge product of $\left.\omega\right|_{U}$ and $\left.\tau\right|_{U}$ gives

$$
\left.(\omega \wedge \tau)\right|_{U}=\sum_{I} \sum_{J} \omega_{I} \tau_{J} d x^{I} \wedge d x^{J} .
$$

Note that $d x^{I} \wedge d x^{J}=0$ if $I$ and $J$ have an index in common. On the other hand, if $I$ and $J$ are disjoint, then rearranging $I \cup J$ on the increasing order and denoting it by $K$, we get $d x^{I} \wedge d x^{J}= \pm d x^{K}$. Consequently,

$$
\left.(\omega \wedge \tau)\right|_{U}=\sum_{K}\left(\sum_{\substack{I \cup J=K \\ I, J \text { disjoint }}} \pm \omega_{I} \tau_{J}\right) d x^{K} .
$$


Since the coefficients of $d x^{K}$ in parentheses are abstract-definable $\mathcal{C}^{r}$ functions on $U$ with $r:=\min \{p, q\} \geq 1$, it follows from Theorem 6.13 that $\omega \wedge \tau$ is an abstract-definable $\mathcal{C}^{r} k$-form on $M$.

The second part of Corollary 6.15 is precisely Proposition 18.3 ([30], p. 202), and its proof is virtually the same.

Corollary 6.15. Let $f_{i}: M \rightarrow R$ be abstract-definable $\mathcal{C}^{p_{i}}$ functions with $p_{i} \geq 2$, $i=1, \ldots, k$. Then the wedge product of their differentials $d f_{1} \wedge \cdots \wedge d f_{k}$ is an abstract-definable $\mathcal{C}^{p} k$-form on $M$ with $p:=\min \left\{p_{i}-1: i=1, \ldots, k\right\}$. Moreover, for any chart $(U, \phi)=\left(U, x^{1}, \ldots, x^{m}\right)$,

$$
d f_{1} \wedge \cdots \wedge d f_{k}=\sum_{I} \frac{\partial\left(f_{1}, \ldots, f_{k}\right)}{\partial\left(x^{i_{1}}, \ldots, x^{i_{k}}\right)} d x^{I} \text { on } U
$$

where $I=\left(1 \leq i_{1}<\cdots<i_{k} \leq m\right)$ and

$$
\frac{\partial\left(f_{1}, \ldots, f_{k}\right)}{\partial\left(x^{i_{1}}, \ldots, x^{i_{k}}\right)}(x):=\operatorname{det}\left[\begin{array}{ccc}
\frac{\partial f_{1}}{\partial x^{i_{1}}}(x) & \cdots & \frac{\partial f_{1}}{\partial x^{i_{k}}}(x) \\
\vdots & \ddots & \vdots \\
\frac{\partial f_{k}}{\partial x^{i_{1}}}(x) & \cdots & \frac{\partial f_{k}}{\partial x^{i_{k}}}(x)
\end{array}\right] .
$$

Proof. The fact that $d f_{1} \wedge \cdots \wedge d f_{k}$ is an abstract-definable $\mathcal{C}^{p} k$-form with $p=$ $\min \left\{p_{1}-1, \ldots, p_{k}-1\right\}$ is an immediate consequence of Example 6.1 and Proposition 6.14. Now, fix a chart $(U, \phi)=\left(U, x^{1}, \ldots, x^{m}\right)$ on $M$. From what we just proved and Theorem 6.13 , it follows that on $U$

$$
d f_{1} \wedge \cdots \wedge d f_{k}=\sum_{J} c_{J} d x^{J}
$$

where $c_{J}$ are abstract-definable $\mathcal{C}^{p}$ functions on $U$. Recall that

$$
\left.\left(d f_{1} \wedge \cdots \wedge d f_{k}\right)\right|_{U}=\left.\left.\left(d f_{1}\right)\right|_{U} \wedge \cdots \wedge\left(d f_{k}\right)\right|_{U},
$$

and according to (6.2) in Example 6.1, each $\left.\left(d f_{i}\right)\right|_{U}$ is given by

$$
\left.\left(d f_{i}\right)\right|_{U}=\sum_{j=1}^{m} \frac{\partial f_{i}}{\partial x^{j}} d x^{j}
$$


Hence, the evaluation of $\left.\left(d f_{i}\right)\right|_{U}$ at $\frac{\partial}{\partial x^{j}}$ equals $\frac{\partial f_{i}}{\partial x^{j}}$. By Proposition A.3 in Appendix A,

$$
\begin{aligned}
\left(\left.\left.\left(d f_{1}\right)\right|_{U} \wedge \cdots \wedge\left(d f_{k}\right)\right|_{U}\right)\left(\frac{\partial}{\partial x^{i_{1}}}, \ldots, \frac{\partial}{\partial x^{i_{k}}}\right) & =\operatorname{det}\left[\begin{array}{ccc}
d f_{1}\left(\frac{\partial}{\partial x^{i_{1}}}\right) & \cdots & d f_{1}\left(\frac{\partial}{\partial x^{i_{k}}}\right) \\
\vdots & \ddots & \vdots \\
d f_{k}\left(\frac{\partial}{\partial x^{i_{1}}}\right) & \cdots & d f_{k}\left(\frac{\partial}{\partial x^{i_{k}}}\right)
\end{array}\right] \\
& =\operatorname{det}\left[\begin{array}{ccc}
\frac{\partial f_{1}}{\partial x^{i_{1}}} & \cdots & \frac{\partial f_{1}}{\partial x^{i_{k}}} \\
\vdots & \ddots & \vdots \\
\frac{\partial f_{k}}{\partial x^{i_{1}}} & \cdots & \frac{\partial f_{k}}{\partial x^{i_{k}}}
\end{array}\right] \\
= & \frac{\partial\left(f_{1}, \ldots, f_{k}\right)}{\partial\left(x^{i_{1}}, \ldots, x^{i_{k}}\right)} .
\end{aligned}
$$

On the other hand,

$$
\left(\sum_{J} c_{J} d x^{J}\right)\left(\frac{\partial}{\partial x^{i_{1}}}, \ldots, \frac{\partial}{\partial x^{i_{k}}}\right)=\sum_{J} c_{J} \delta_{I}^{J}=c_{I}
$$

where in the second identity we used Lemma A.4 in Appendix A. From (6.11), the result therefore is obtained.

The transition formula for abstract-definable $\mathcal{C}^{p} k$-forms on $M$ is an immediate consequence of Corollary 6.15. This is stated in the classical context in ([30], p. 202).

Corollary 6.16. Assume $p \geq 2$. If $\left(U, x^{1}, \ldots, x^{m}\right)$ and $\left(V, y^{1}, \ldots, y^{m}\right)$ are two overlapping charts on $M$, then

$$
d y^{j_{1}} \wedge \cdots \wedge d y^{j_{k}}=\sum_{1 \leq i_{1}<\ldots<i_{k} \leq m} \frac{\partial\left(y^{j_{1}}, \ldots, y^{j_{k}}\right)}{\partial\left(x^{i_{1}}, \ldots, x^{i_{k}}\right)} d x^{i_{1}} \wedge \cdots \wedge d x^{i_{k}} .
$$

Proof. By taking each $f_{l}$ in Corollary 6.15 to be $y^{j_{l}}$, the result follows.

Consider an abstract-definable $\mathcal{C}^{p}$ map $F: N \rightarrow M$ and a map $\omega: M \rightarrow \bigwedge^{k} T^{*} M$ given by $x \mapsto\left(x, \omega_{x}\right)$. The pullback of $\omega$ by $F$ is the map $F^{*} \omega: N \rightarrow \bigwedge^{k} T^{*} N$ defined as

$$
x \mapsto\left(x,\left(F^{*} \omega\right)_{x}\right),
$$

where $\left(F^{*} \omega\right)_{x}: T_{x} N \times \cdots \times T_{x} N \rightarrow R$ is the following $k$-linear function

$$
\left(F^{*} \omega\right)_{x}\left(v_{1}, \ldots, v_{k}\right):=\omega_{F(x)}\left(d_{x} F v_{1}, \ldots, d_{x} F v_{k}\right)
$$


As in Remark 6.4, the set of maps $\omega: M \rightarrow \wedge^{k} T^{*} M$ such that $\pi \circ \omega=\operatorname{id}_{M}$, where $\pi: \wedge^{k} T^{*} M \rightarrow M$ is the projection given in Proposition 6.8, equipped with analogous operations to those in (6.6), forms an $R$-vector space and a $\mathcal{F}(M)$ module, where $\mathcal{F}(M)$ denotes the ring of $R$-valued functions on $M$. This leads us to the following.

Lemma 6.17 (Linearity of the pullback). Let $F: N \rightarrow M$ be an abstract-definable $\mathcal{C}^{p}$ map, $g: M \rightarrow R$ an abstract-definable $\mathcal{C}^{p}$ function, and let $\omega, \tau: M \rightarrow \bigwedge^{k} T^{*} M$ be maps given by $x \mapsto\left(x, \omega_{x}\right)$ and $x \mapsto\left(x, \tau_{x}\right)$, respectively. Then,

(1) $F^{*}(\omega+\tau)=F^{*} \omega+F^{*} \tau$;

(2) $F^{*}(g \omega)=\left(F^{*} g\right)\left(F^{*} \omega\right)$. In particular, when $g \equiv a \in R$ is a constant function on $M, F^{*}(a \omega)=a\left(F^{*} \omega\right)$.

Proof. Similar to that of Lemma 6.5.

Lemma 6.18 (Pullback of the wedge product). If $F: N \rightarrow M$ is an abstractdefinable $\mathcal{C}^{p}$ map and $\omega, \tau$ are maps given by $x \mapsto\left(x, \omega_{x}\right): M \rightarrow \wedge^{k} T^{*} M$ and $x \mapsto \bigwedge^{l} T^{*} M$, respectively, then

$$
F^{*}(\omega \wedge \tau)=\left(F^{*} \omega\right) \wedge\left(F^{*} \tau\right)
$$

Proof. It suffices to show that for any $x \in N,\left(F^{*}(\omega \wedge \tau)\right)_{x}=\left(F^{*} \omega\right)_{x} \wedge\left(F^{*} \tau\right)_{x}$. Let $v_{1}, \ldots, v_{k+l}$ be vectors in $T_{x} N$. Then,

$$
\begin{aligned}
& \left(F^{*}(\omega \wedge \tau)\right)_{x}\left(v_{1}, \ldots, v_{k+l}\right)= \\
& (\omega \wedge \tau)_{F(x)}\left(d_{x} F v_{1}, \ldots, d_{x} F v_{k+1}\right)= \\
& \left(\omega_{F(x)} \wedge \tau_{F(x)}\right)\left(d_{x} F v_{1}, \ldots, d_{x} F v_{k+1}\right)= \\
& \frac{1}{k ! l !} \sum_{\sigma \in \mathbb{S}_{k+l}}(\operatorname{sgn} \sigma) \omega_{F(x)}\left(d_{x} F v_{\sigma(1)}, \ldots, d_{x} F v_{\sigma(k)}\right) \tau_{F(x)}\left(d_{x} F v_{\sigma(k+1)}, \ldots, d_{x} F v_{\sigma(k+l)}\right)= \\
& \frac{1}{k ! l !} \sum_{\sigma \in \mathbb{S}_{k+l}}(\operatorname{sgn} \sigma)\left(F^{*} \omega\right)_{x}\left(v_{\sigma(1)}, \ldots, v_{\sigma(k)}\right)\left(F^{*} \tau\right)_{x}\left(v_{\sigma(k+1)}, \ldots, v_{\sigma(k+l)}\right)= \\
& \left(\left(F^{*} \omega\right)_{x} \wedge\left(F^{*} \tau\right)_{x}\right)\left(v_{1}, \ldots, v_{k+l}\right) .
\end{aligned}
$$

(See Appendix A for the definition of wedge product in multilinear algebra used in the third and last identities above.) 
We end this section with the analogue of Proposition 6.6 for abstract-definable $k$-forms. It is also the abstract-definable version of Proposition 19.7 ([30], p. 215) whose proof is fully mimicked here.

Proposition 6.19. The pullback $F^{*} \omega$ of an abstract-definable $\mathcal{C}^{p} k$-form $\omega$ on $M$ under an abstract-definable $\mathcal{C}^{p}$ map $F: N \rightarrow M$ with $p \geq 2$ is an abstract-definable $\mathcal{C}^{p-1} k$-form on $N$.

Proof. By Theorem 6.13, it suffices to show that for each $x \in N$ there is a chart $(V, \psi)=\left(V, y^{1}, \ldots, y^{n}\right)$ on $N$ at $x$ in which $F^{*} \omega$ is given by $\sum_{I} \tau_{I} d y^{I}$, with $\tau_{I}: V \rightarrow R$ abstract-definable $\mathcal{C}^{p-1}$ functions. Indeed, let $x \in N$ and $(U, \phi)=\left(U, x^{1}, \ldots, x^{m}\right)$ a chart on $M$ at $F(x)$. Since $F$ is continuous, there is a chart $(V, \psi)$ lying in an abstract-definable $\mathcal{C}^{p}$ atlas over $N$, which is $\sim$-equivalent to $\mathcal{B}$, satisfying $F(x) \in F(V) \subseteq U$. From Theorem 6.13, it follows that on $U$ the map $\omega$ is written as $\omega=\sum_{I} \omega_{I} d x^{I}$ with $\omega_{I}: U \rightarrow R$ abstract-definable $\mathcal{C}^{p}$ functions. Set $F^{i}:=\left.x^{i} \circ F\right|_{V}$ for each $i=1, \ldots, m$ and note that $F^{i}$ is an abstract-definable $\mathcal{C}^{p}$ function on $V$. Hence,

$$
\begin{aligned}
\left.\left(F^{*} \omega\right)\right|_{V} & =\left.\sum_{I}\left(\left.F\right|_{V} ^{*} \omega_{I}\right) F\right|_{V} ^{*}\left(d x^{i_{1}} \wedge \cdots \wedge d x^{i_{k}}\right) \\
& =\sum_{I}\left(\left.\omega_{I} \circ F\right|_{V}\right) d\left(\left.x^{i_{1}} \circ F\right|_{V}\right) \wedge \cdots \wedge d\left(\left.x^{i_{k}} \circ F\right|_{V}\right) \\
& =\sum_{I}\left(\left.\omega_{I} \circ F\right|_{V}\right) d F^{1} \wedge \cdots \wedge d F^{k} \\
& =\sum_{I, J}\left(\left.\omega_{I} \circ F\right|_{V}\right) \frac{\partial\left(F^{1}, \ldots, F^{k}\right)}{\partial\left(y^{j_{1}}, \ldots, y^{j_{k}}\right)} d y^{J},
\end{aligned}
$$

where the above identities result from Lemmas 6.17, 6.18, and 6.5, and also from Corollary 6.15. Finally, observe that the coefficients of each $d y^{J}$ are abstractdefinable $\mathcal{C}^{p-1}$ functions on $V$. 



\section{Chapter 7}

\section{Exterior derivative}

As we know from the standard and non-standard context, the class $\mathcal{C}^{p}$ of differentiability for $p<\infty$ is not closed under differentiation. In walking the path towards a de Rham-like cohomology theory for o-minimal manifolds, there was no need of this closure condition up to now, and therefore we were allowed to work within the general setting of an o-minimal expansion of a real closed field. However, since we aim to construct cochain complexes whose objects are sets of abstractdefinable forms by following the lines of the classical de Rham cohomology theory, we must establish exterior derivative, and this requires such a closure condition on differentiability. So, we turn our attention to abstract-definable $\mathcal{C}^{\infty}$ manifolds, and by virtue of [16] we need to restrict ourselves to an o-minimal expansion of the real field which possesses $\mathcal{C}^{\infty}$ cell decomposition. Recall that in building up abstract-definable $\mathcal{C}^{p}$ partitions of unity we made heavy use of results, specifically Theorem 3.2 by A. Thamrongthanyalak and Lemma 3.3, in the definable context that "a priori" only hold for $p<\infty$. Nonetheless, if in addition to admitting a smooth cell decomposition the o-minimal expansion of the real field defines the exponential function, then we have in hand Lemma 4.6 and Theorem 5.3 in [11], which are abstract-definable smooth versions of Theorem 3.4 and Proposition 3.7, respectively. Therefore, proceeding just like in the proofs of Propositions 3.6 and 3.8, and Corollary 3.9, we obtain the abstract-definable $\mathcal{C}^{\infty}$ analogues of these results. Namely,

Proposition 7.1. Let $\{V, W\}$ be an abstract-definable open cover of $M$. Then, there are abstract-definable $\mathcal{C}^{\infty}$ functions $f_{V}, f_{W}: M \rightarrow R$ such that:

(1) $f_{V}, f_{W} \geq 0$; 
(2) $\operatorname{supp}\left(f_{V}\right) \subseteq V$ and $\operatorname{supp}\left(f_{W}\right) \subseteq W$;

(3) $f_{V}+f_{W}=1$.

Proposition 7.2. Let $F$ be an abstract-definable closed set in $M$, and $U$ an abstract-definable open set in $M$ containing $F$. Then, there exists an abstractdefinable $\mathcal{C}^{\infty}$ function $\rho: M \rightarrow R$ so that

(1) $0 \leq \rho \leq 1$

(2) $\left.\rho\right|_{F}=1$;

(3) $\operatorname{supp}(\rho) \subseteq U$.

Corollary 7.3. For any abstract-definable open subset $U \subseteq M$, there is an abstractdefinable $\mathcal{C}^{\infty}$ nonnegative function $\rho: M \rightarrow R$ such that

(1) $\left.\rho\right|_{V}=1$, for some abstract-definable open set $V \subseteq U$;

(2) $\operatorname{supp}(\rho) \subseteq U$.

The function $\rho$ is called an abstract-definable $\mathcal{C}^{\infty}$ bump function supported in $U$.

In view of this, we fix from now on an o-minimal expansion $\mathcal{R}$ of the real field $\mathbb{R}$ that admits smooth cell decomposition and defines the exponential function. By "definable" we mean "definable in $\mathcal{R}$ with parameters in $\mathbb{R}$ ".

In the present and later chapters, our focus will be on abstract-definable $\mathcal{C}^{p} k$-forms with $p=\infty$. In addition to all we developed so far for abstract-definable $\mathcal{C}^{p}$ manifolds with $p<\infty$ holding for the case $p=\infty$, we have clear improvements. For instance, the pullback $F^{*} \omega$ of an abstract-definable $\mathcal{C}^{\infty} k$-form $\omega$ on an abstractdefinable $\mathcal{C}^{\infty}$ manifold $M$ under an abstract-definable $\mathcal{C}^{\infty}$ map $F: N \rightarrow M$ is an abstract-definable $\mathcal{C}^{\infty} k$-form on the abstract-definable $\mathcal{C}^{\infty}$ manifold $N$. That is, there is no decreasing in the differentiability class of $F^{*} \omega$.

Instead of restating all results specially from Chapter 6 for the case $p=\infty$, we will refer to them as if they were asserted and proved for $p=\infty$.

In Section 1 of this chapter, we introduce the notion of exterior derivative and local operator as in the classical setting. In Section 2, we prove the existence 
and uniqueness of the exterior derivative for local abstract-definable $\mathcal{C}^{\infty}$ forms, more specifically defined on a chart. We then extend in Section 3 such a result for global abstract-definable $\mathcal{C}^{\infty}$ forms, and end the chapter by showing that the exterior derivative and the pullback commute. We follow closely Section 19 of [30].

Throughout the chapter, unless otherwise stated, $(M, \mathcal{A})$ and $(N, \mathcal{B})$ denote abstractdefinable $\mathcal{C}^{\infty}$ manifolds of dimensions $m$ and $n$, respectively.

\subsection{Local operators}

For each $k$, let $\Omega^{k}(M)$ denote the set of all abstract-definable $\mathcal{C}^{\infty} k$-forms. This set equipped with the pointwise sum and scalar multiplication of maps forms an $\mathbb{R}$-vector space. Take $\Omega^{*}(M)$ to be the $\mathbb{R}$-vector space given by the the direct sum

$$
\Omega^{*}(M):=\bigoplus_{j=0}^{m} \Omega^{k}(M) .
$$

As pointed out in Appendix A, this means that every element of $\Omega^{*}(M)$ can be uniquely written as a sum $\sum_{k=0}^{m} \omega_{k}$ with $\omega_{k} \in \Omega^{k}(M)$. With the wedge product, the vector space $\Omega^{*}(M)$ becomes an anticommutative graded algebra (see Appendix A for this notion), where the grading is the degree of the abstract-definable $\mathcal{C}^{k}$ forms on $M$. Also, from Lemmas 6.17 and 6.18, if $F: M \rightarrow N$ is an abstract-definable $\mathcal{C}^{\infty}$ map then the pullback map $F^{*}: \Omega^{*}(N) \rightarrow \Omega^{*}(M)$ is a homomorphism of graded algebras.

Inspired by Definitions 19.1 and 19.2 ([30], pp. 210 and 211), we introduce the notions of exterior derivative and local operator on abstract-definable $\mathcal{C}^{\infty}$ forms as follows.

Definition 7.4. An exterior derivative on $M$ is an $\mathbb{R}$-linear operator $D: \Omega^{*}(M) \rightarrow$ $\Omega^{*}(M)$ satisfying the conditions:

(1) $D$ is an antiderivation of degree 1 (see Appendix A for this notion);

(2) $D \circ D=0$;

(3) if $f \in \Omega^{0}(M)$ (that is, $f: M \rightarrow \mathbb{R}$ is an abstract-definable $\mathcal{C}^{\infty}$ function), then $D f=d f$, where $d f$ denotes the differential of $f$ (see Example 6.1). 
Definition 7.5. An $\mathbb{R}$-linear operator $d: \Omega^{*}(M) \rightarrow \Omega^{*}(M)$ is called local if it has the property: for all $k \geq 0$, if $\omega$ is an abstract-definable $\mathcal{C}^{\infty} k$-form on $M$ in such a way that $\left.\omega\right|_{U}=0$ for some abstract-definable open subset $U$ of $M$, then $d \omega=0$ on $U$.

Equivalently, a linear operator $d: \Omega^{*}(M) \rightarrow \Omega^{*}(M)$ is local if for all $k \geq 0$, whenever two abstract-definable $\mathcal{C}^{\infty} k$-forms $\omega$ and $\tau$ on $M$ agree on an abstractdefinable open set $U$, then $d \omega=d \tau$ on $U$.

The following is the abstract-definable version of Proposition 19.3 ([30], p. 212).

Proposition 7.6. Every antiderivation $D$ on $\Omega^{*}(M)$ is a local operator.

Proof. Let $\omega$ be an abstract-definable $\mathcal{C}^{\infty} k$-form on $M$, and assume that $\omega$ is identically 0 on an abstract-definable open set $U \subseteq M$. It suffices to show that for an arbitrary point $x \in U,(D \omega)_{x}=0$. Let $\rho: M \rightarrow \mathbb{R}$ be an abstract-definable $\mathcal{C}^{\infty}$ bump function at $x$ supported in $U$, i.e., $\rho$ is an abstract-definable $\mathcal{C}^{\infty}$ nonnegative function such that equals 1 in an abstract-definable neighborhood of $x$, and its support $\operatorname{supp}(\rho)$ is contained in $U$. Consequently, $\rho \cdot \omega=0$ on $M$. Applying the antiderivation property of $D$ to the abstract-definable $\mathcal{C}^{\infty} k$-form $\rho \omega$ on $M$, we obtain

$$
\begin{aligned}
0=D(0)=D(\rho \omega) & =(D \rho) \wedge \omega+(-1)^{0} \rho \wedge D \omega \\
& =d \rho \wedge \omega+\rho D \omega
\end{aligned}
$$

where $d \rho$ denotes the differential of $\rho$. The evaluation of both sides at $x \in U$ gives

$$
0=d_{x} \rho \wedge \omega_{x}+\rho(x)(D \omega)_{x}=(D \omega)_{x}
$$

with the second equality following from the facts $\omega_{x}=0$ and $\rho(x)=1$.

\subsection{Existence and uniqueness of a local exterior derivative}

We prepare in this section for the establishment of an exterior derivative on abstract-definable forms. This is done in the lines of Subsection 19.1 ([30], p. 211). Before, we fix some notation. 
Notation. Throughout the chapter, unless otherwise specified, I denotes a $k$-tuple $\left(i_{1}, \ldots, i_{k}\right)$, where $1 \leq i_{1}<\cdots<i_{k} \leq m, d x^{I}$ is short for $d x^{i_{1}} \wedge \cdots \wedge d x^{i_{k}}$, and $\left.d x^{I}\right|_{x}$ designates $\left.\left.d x^{i_{1}}\right|_{x} \wedge \cdots \wedge d x^{i_{k}}\right|_{x}$.

Proposition 7.7. Let $\left(U, x^{1}, \ldots, x^{m}\right)$ be a chart on $M$. There exists a unique exterior derivative $d_{U}$ on $U$.

Proof. Recall from Lemma 6.12 that any abstract-definable $\mathcal{C}^{\infty} k$-form $\tau$ on $U$ is written as $\sum_{I} \tau_{I} d x^{I}$, with $\tau_{I}$ an abstract-definable $\mathcal{C}^{\infty}$ function on $U$. Define a map $d_{U}: \Omega^{*}(U) \rightarrow \Omega^{*}(U)$ in such a way that its restriction to each $\Omega^{k}(U)$ is given by

$$
d_{U}(\tau):=\sum_{I}\left(\sum_{j} \frac{\partial \tau_{I}}{\partial x^{j}} d x^{j}\right) \wedge d x^{I},
$$

In the case $k=0,(7.1)$ means that $d_{U}(\tau)$ is the differential of $\tau$. Since $\frac{\partial}{\partial x^{i}}$ is $\mathbb{R}$-linear with respect to the abstract-definable $\mathcal{C}^{p}$ functions on $U$ (see Section 1 of Chapter 4$)$, the map $d_{U}$ is linear on $\Omega^{k}(U)$, and hence on $\Omega^{*}(U)$. Let us now check for $d_{U}$ the conditions (1)-(3) as in Definition 7.4. Condition (3) is precisely the definition of $d_{U}$ on $\Omega^{0}(U)$, as we just pointed out. Let $\sum_{I} \alpha_{I} d x^{I}$ and $\sum_{J} \beta_{J} d x^{J}$ be abstract-definable $\mathcal{C}^{\infty} k$ - and $l$-forms on $U$, respectively.

(1) We claim that

$$
d_{U}\left(\alpha_{I} d x^{I} \wedge \beta_{J} d x^{J}\right)=d_{U}\left(\alpha_{I} d x^{I}\right) \wedge\left(\beta_{J} d x^{J}\right)+(-1)^{k}\left(\alpha_{I} d x^{I}\right) \wedge d_{U}\left(\beta_{J} d x^{J}\right),
$$

for each $I$ and $J$. Indeed, by definition

$$
\begin{aligned}
d_{U}\left(\alpha_{I} d x^{I} \wedge \beta_{J} d x^{J}\right) & =d_{U}\left(\alpha_{I} \beta_{J} d x^{I} \wedge d x^{J}\right) \\
& =\sum_{i} \frac{\partial\left(\alpha_{I} \beta_{J}\right)}{\partial x^{i}} d x^{i} \wedge d x^{I} \wedge d x^{J} \\
& =\sum_{i} \frac{\partial \alpha_{I}}{\partial x^{i}} \beta_{J} d x^{i} \wedge d x^{I} \wedge d x^{J}+\sum_{i} \alpha_{I} \frac{\partial \beta_{J}}{\partial x^{i}} d x^{i} \wedge d x^{I} \wedge d x^{J} .
\end{aligned}
$$


By the anticommutativity of the wedge product, we get $d x^{i} \wedge d x^{I}=(-1)^{k} d x^{I} \wedge d x^{i}$, and hence the above equality becomes

$$
\begin{aligned}
d_{U}\left(\alpha_{I} d x^{I} \wedge \beta_{J} d x^{J}\right) & =\sum_{i} \frac{\partial \alpha_{I}}{\partial x^{i}} \beta_{J} d x^{i} \wedge d x^{I} \wedge d x^{J}+(-1)^{k} \sum_{i} \alpha_{I} d x^{I} \wedge \frac{\partial \beta_{J}}{\partial x^{i}} d x^{i} \wedge d x^{J} \\
& =\sum_{i} \frac{\partial \alpha_{I}}{\partial x^{i}} \beta_{J} d x^{i} \wedge d x^{I} \wedge d x^{J}+(-1)^{k} \alpha_{I} d x^{I} \wedge\left(\sum_{i} \frac{\partial \beta_{J}}{\partial x^{i}} d x^{i}\right) \wedge d x^{J} \\
& =d_{U}\left(\alpha_{I} d x^{I}\right) \wedge\left(\beta_{J} d x^{J}\right)+(-1)^{k}\left(\alpha_{I} d x^{I}\right) \wedge d_{U}\left(\beta_{J} d x^{J}\right) .
\end{aligned}
$$

Since $d_{U}$ is linear, the claimed identity holds for $\sum_{I} \alpha_{I} d x^{I}$ and $\sum_{J} \beta_{J} d x^{J}$, and consequently $d_{U}: \Omega^{*}(U) \rightarrow \Omega^{*}(U)$ is an antiderivation.

(2) For each $I$,

$$
\begin{aligned}
d_{U}\left(d_{U}\left(\alpha_{I} d x^{I}\right)\right) & =d_{U}\left(\sum_{i} \frac{\partial \alpha_{I}}{\partial x^{i}} d x^{i} \wedge d x^{I}\right) \\
& =\sum_{j, i} \frac{\partial^{2} \alpha_{I}}{\partial x^{j} \partial x^{i}} d x^{j} \wedge d x^{i} \wedge d x^{I} .
\end{aligned}
$$

Note that if $i=j$, then $d x^{i} \wedge d x^{j}=0$, and if $i \neq j$, then $d x^{i} \wedge d x^{j}=-d x^{j} \wedge d x^{i}$. But, by the fact that $\frac{\partial^{2} \alpha_{I}}{\partial x^{i} \partial x^{j}}$ equals $\frac{\partial^{2} \alpha_{I}}{\partial x^{j} \partial x^{i}}$, the terms with $i \neq j$ cancel each other. Hence, $\left(d_{U} \circ d_{U}\right)\left(\alpha_{I} d x^{I}\right)=0$. Again, because $d_{U}$ is linear, $d_{U}^{2}\left(\sum_{I} \alpha_{I} d x^{I}\right)=0$. Therefore, the linear map $d_{U}: \Omega^{*}(U) \rightarrow \Omega^{*}(U)$ satisfies $d_{U} \circ d_{U}=0$.

Let us now prove the uniqueness of $d_{U}$. Suppose $D_{U}$ is an exterior derivative on $U$. For any $\tau:=\sum_{I} \tau_{I} d x^{I} \in \Omega^{k}(U)$, we obtain from conditions (1), (3) and (2) the following

$$
\begin{aligned}
D_{U}(\tau) & =\sum_{I}\left(D_{U}\left(\tau_{I}\right) \wedge d x^{I}+\tau_{I} D_{U}\left(d x^{I}\right)\right) \\
& =\sum_{I}\left(D_{U}\left(\tau_{I}\right) \wedge d x^{I}+\tau_{I} D_{U}\left(D x^{I}\right)\right) \\
& =\sum_{I}\left(D_{U}\left(\tau_{I}\right) \wedge d x^{I}+\tau_{I}\left(\sum_{j=1}^{k}(-1)^{j-1} D x^{i_{1}} \wedge \cdots \wedge D\left(D x^{i_{j}}\right) \wedge \cdots \wedge D x^{i_{k}}\right)\right) \\
& =\sum_{I} D_{U}\left(\tau_{I}\right) \wedge d x^{I} \\
& =\sum_{I}\left(\sum_{i} \frac{\partial \tau_{I}}{\partial x^{i}} d x^{i}\right) \wedge d x^{I} .
\end{aligned}
$$

Therefore, $D_{U}(\tau)=d_{U}(\tau)$. 


\subsection{Existence and uniqueness of a global exterior derivative}

Before we state and prove the main result of the chapter, we set up a lemma concerning extension of local abstract-definable $k$-forms.

Lemma 7.8. Let $\left(U, x^{1}, \ldots, x^{m}\right)$ be a chart on $M$, and $\omega$ an abstract-definable $\mathcal{C}^{\infty} k$-form on $U$. Then, there is an abstract-definable $\mathcal{C}^{\infty} k$-form $\widetilde{\omega}$ on $M$ and an abstract-definable nonempty open set $V \subseteq U$ such that $\left.\widetilde{\omega}\right|_{V}=\left.\omega\right|_{V}$.

Proof. Write $\omega$ as $\sum_{I} \omega_{I} d x^{I}$, where $\omega_{I}$ are abstract-definable $\mathcal{C}^{\infty}$ functions on $U$. Consider an abstract-definable $\mathcal{C}^{\infty}$ bump function $\rho: M \rightarrow \mathbb{R}$ supported in $U$, and let $V \subseteq U$ be the abstract-definable nonempty open set on which $\rho$ is identically 1. Then, defining $\widetilde{\omega}_{I}: M \rightarrow \mathbb{R}$ as

$$
\widetilde{\omega}_{I}(z):=\left\{\begin{array}{ll}
\rho(z) \omega_{I}(z), & \text { for } z \in U \\
0, & \text { for } z \notin U
\end{array},\right.
$$

it follows that $\widetilde{\omega}_{I}$ is an abstract-definable $\mathcal{C}^{\infty}$ function, and on $V$ both functions $\widetilde{\omega}_{I}$ and $\omega_{I}$ coincide. Similarly, we obtain abstract-definable $\mathcal{C}^{\infty}$ functions $\widetilde{x}^{i}: M \rightarrow \mathbb{R}$ extending $\left.x^{i}\right|_{V}$. Now, set

$$
\widetilde{\omega}:=\sum_{I} \widetilde{\omega}_{I} d \widetilde{x}^{I}
$$

By Corollary 6.15 and by the fact that $\Omega^{k}(M)$ is a $\mathcal{C}^{\infty}(M)$-module, where $\mathcal{C}^{\infty}(M)$ denotes the ring of all abstract-definable $\mathcal{C}^{\infty}$ functions on $M, \widetilde{\omega}$ is an abstractdefinable $\mathcal{C}^{\infty} k$-form on $M$. Finally, note that $\left.\widetilde{\omega}\right|_{V}=\left.\left.\left.\sum_{I} \widetilde{\omega}_{I}\right|_{V} d \widetilde{x}^{i_{1}}\right|_{V} \wedge \cdots \wedge d \widetilde{x}^{i_{k}}\right|_{V}$, and hence equals $\left.\omega\right|_{V}$.

The following is an abstract-definable adaptation of the Subsections 19.3 and 19.4 ([30], pp. 212 and 213).

Theorem 7.9. There exists an exterior derivative $d: \Omega^{*}(M) \rightarrow \Omega^{*}(M)$ which is uniquely determined by the conditions (1)-(3) in Definition 7.4 .

Proof. For each $k$, let $\left.d\right|_{\Omega^{k}(M)}: \Omega^{k}(M) \rightarrow \Omega^{k+1}(M)$ be the map

$$
\omega \mapsto\left(x \mapsto\left(x,\left(\left.d_{U} \omega\right|_{U}\right)_{x}\right)\right)
$$


where $\left(U, x^{1}, \ldots, x^{m}\right)$ is a chart on $M$ at $x$, and $d_{U}$ is the map given by (7.1) in the proof of Proposition 7.7. Hence, writing $\left.\omega\right|_{U}$ as $\sum_{I} \omega_{I} d x^{I}$ with $\omega_{I}: U \rightarrow \mathbb{R}$ abstract-definable $\mathcal{C}^{\infty}$ functions, we have

$$
\left(\left.d_{U} \omega\right|_{U}\right)_{x}=\left.\sum_{I}\left(\left.\sum_{i} \frac{\partial \omega_{I}}{\partial x^{i}}(x) d x^{i}\right|_{x}\right) \wedge d x^{I}\right|_{x}
$$

In order to conclude that $\left.d\right|_{\Omega^{k}(M)}$ is well-defined we must verify that $\left(\left.d\right|_{\Omega^{k}(M)} \omega\right)_{x}$ is independent of the chart $U$ containing $x$. Let $\left(V, y^{1}, \ldots, y^{m}\right)$ be another chart on $M$ at $x$, and let $\sum_{J} \omega_{J}^{\prime} d y^{J}$ be the restriction $\left.\omega\right|_{V}$, where $\omega_{J}^{\prime}$ are abstract-definable $\mathcal{C}^{\infty}$ functions on $V$. Recall from Proposition 7.7 that there is a unique exterior derivative $d_{U \cap V}$ on $U \cap V$. Also, on $U \cap V$

$$
\sum_{I} \omega_{I} d x^{I}=\sum_{J} \omega_{J}^{\prime} d y^{J}
$$

Then, computing $d_{U \cap V}$ on both sides of the above equality, we obtain in particular

$$
\left.\sum_{I}\left(\left.\sum_{i} \frac{\partial \omega_{I}}{\partial x^{i}}(x) d x^{i}\right|_{x}\right) \wedge d x^{I}\right|_{x}=\left.\sum_{J}\left(\left.\sum_{i} \frac{\partial \omega_{J}^{\prime}}{\partial y^{i}}(x) d y^{i}\right|_{x}\right) \wedge d y^{J}\right|_{x} .
$$

In other words, $\left(\left.d_{U} \omega\right|_{U}\right)_{x}=\left(\left.d_{V} \omega\right|_{V}\right)_{x}$.

Define $d: \Omega^{*}(M) \rightarrow \Omega^{*}(M)$ by

$$
d\left(\omega_{0}+\cdots+\omega_{m}\right):=\left.d\right|_{\Omega^{0}(M)}\left(\omega_{0}\right)+\cdots+\left.d\right|_{\Omega^{m}(M)}\left(\omega_{m}\right) .
$$

Each map $\left.d\right|_{\Omega^{k}(M)}$ is linear, since for $\omega, \tau \in \Omega^{k}(M), a \in \mathbb{R}$, and for any $x \in M$

$$
\left(\left.d\right|_{\Omega^{k}(M)}(\omega+a \tau)\right)_{x}=\left(\left.d_{U}(\omega+a \tau)\right|_{U}\right)_{x}
$$

where $\left(U, x^{1}, \ldots, x^{m}\right)$ is a chart on $M$ at $x$; by Proposition $7.7, d_{U}$ is linear, and hence

$$
\left(\left.d_{U}(\omega+a \tau)\right|_{U}\right)_{x}=\left(\left.d\right|_{\Omega^{k}(M)} \omega\right)_{x}+a\left(\left.d\right|_{\Omega^{k}(M)} \tau\right)_{x} .
$$

Consequently, $d$ is linear. In a similar way, in order to verify the conditions (1)-(3) in Definition 7.4 for $d$, it suffices to check them at each point of $M$. But, such a verification reduces to showing them for $d_{U}$, which are ensured by Proposition 7.7. In summary, $d$ is an exterior derivation.

For the uniqueness of $d$, suppose $D: \Omega^{*}(M) \rightarrow \Omega^{*}(M)$ is an exterior derivation. 
We will show that $D \omega=d \omega$, for any $\omega \in \Omega^{k}(M)$ with $k \in\{0, \ldots, m\}$. It suffices then to check $(D \omega)_{x}=(d \omega)_{x}$, for each $x \in M$.

Claim 7.10. For any abstract-definable $\mathcal{C}^{\infty}$ functions $f_{1}, \ldots, f_{k}: M \rightarrow \mathbb{R}$,

$$
D\left(d f_{1} \wedge \cdots \wedge d f_{k}\right)=0
$$

Proof of the Claim. First, observe that the condition (3) in the definition of an exterior derivative implies that for any abstract-definable $\mathcal{C}^{\infty}$ function $f$ on $M$, $D f$ agrees with $d f$ (the differential of $f$ ). As a consequence, if $f_{1}, \ldots, f_{k}: M \rightarrow \mathbb{R}$ are arbitrary abstract-definable $\mathcal{C}^{\infty}$ functions, then

$$
\begin{aligned}
D\left(d f_{1} \wedge \cdots \wedge d f_{k}\right) & =D\left(D f_{1} \wedge \cdots \wedge D f_{k}\right) \\
& =\sum_{i=1}^{k}(-1)^{i-1} D f_{1} \wedge \cdots \wedge D\left(D f_{i}\right) \wedge \cdots \wedge D f_{k} \\
& =0
\end{aligned}
$$

where the second identity follows from the fact that $D$ is an antiderivation, and the last from the condition $D^{2}=0$.

Now, consider an abstract-definable $\mathcal{C}^{\infty} k$-form $\omega$ on $M, x$ a point in $M$, and $\left(U, x^{1}, \ldots, x^{m}\right)$ a chart on $M$ at $x$. Write $\left.\omega\right|_{U}$ as $\sum_{I} \omega_{I} d x^{I}$, with $\omega_{I}: U \rightarrow \mathbb{R}$ abstract-definable $\mathcal{C}^{\infty}$ functions. According to Lemma 7.8, there is an abstractdefinable $\mathcal{C}^{\infty} k$-form $\widetilde{\omega}$ on $M$ given by $\sum_{I} \widetilde{\omega}_{I} d \widetilde{x}^{I}$, where $\widetilde{\omega}_{I}$ and $\widetilde{x}^{i}$ are abstractdefinable $\mathcal{C}^{\infty}$ functions on $M$ that agree with $\omega_{I}$ and $x^{i}$, respectively, on an abstract-definable open neighborhood $V \subseteq U$ containing $x$. Since $\left.\widetilde{\omega}\right|_{V}=\left.\omega\right|_{V}$ and $D$ is a local operator on $M$ (see Proposition 7.6), D $\widetilde{\omega}=D \omega$ on $V$. In particular, we get $(D \omega)_{x}=(D \widetilde{\omega})_{x}$. From the condition (1) and from Claim 7.10, the 
second and third identities below follow

$$
\begin{aligned}
(D \widetilde{\omega})_{x} & =\left(D \sum_{I} \widetilde{\omega}_{I} d \widetilde{x}^{I}\right)_{x} \\
& =\left(\sum_{I} D \widetilde{\omega}_{I} \wedge d \widetilde{x}^{I}+\sum_{I} \widetilde{\omega}_{I} \wedge D\left(d \widetilde{x}^{I}\right)\right)_{x} \\
& =\left(\sum_{I} d \widetilde{\omega}_{I} \wedge d \widetilde{x}^{I}\right)_{x} \\
& =\left(\left.\left.\sum_{I} d \widetilde{\omega}_{I}\right|_{V} \wedge d \widetilde{x}^{I}\right|_{V}\right)_{x}
\end{aligned}
$$

Since $\left.d \widetilde{\omega}_{I}\right|_{V}=\left.d \omega_{I}\right|_{V}$ and $\left.d \widetilde{x}^{I}\right|_{V}=\left.d x^{I}\right|_{V}$,

$$
(D \widetilde{\omega})_{x}=\left(\left.\left.\sum_{I} d \omega_{I}\right|_{V} \wedge d x^{I}\right|_{V}\right)_{x}=\left(\left.d_{U} \omega\right|_{U}\right)_{x}=(d \omega)_{x} .
$$

We end this section with the property that the pullback under an abstract-definable $\mathcal{C}^{\infty}$ map commutes with the exterior derivative. The proof here is virtually the same as that of Proposition 19.5 ([30], p. 214).

Proposition 7.11. Consider an abstract-definable $\mathcal{C}^{\infty}$ map $F: N \rightarrow M$, and let $\omega \in \Omega^{k}(M)$. Then, $d\left(F^{*} \omega\right)=F^{*} d \omega$.

Proof. We will only consider the proposition for $k>0$, since the case $k=0$ is Lemma 6.5(1). Let $\omega \in \Omega^{k}(M)$. Note that in view of the definition of $d$ it suffices to check the statement at any point $x \in N$, which in turn reduces to verifying it on charts $\left(U, x^{1}, \ldots, x^{m}\right)$ on $M$ at $F(x)$ and $\left(V, y^{i_{1}}, \ldots, y^{i_{k}}\right)$ on $N$ at $x$. On $U, \omega$ is written as $\omega=\sum_{I} \omega_{I} d x^{i_{1}} \wedge \cdots \wedge d x^{i_{k}}$ with $\omega_{I}: M \rightarrow \mathbb{R}$ abstract-definable $\mathcal{C}^{\infty}$ functions, and by Proposition 6.14 and Lemma 6.5(1), we get

$$
\begin{aligned}
F^{*} \omega & =\sum_{I}\left(F^{*} \omega_{I}\right) F^{*} d x^{i_{1}} \wedge \cdots \wedge F^{*} d x^{i_{k}} \\
& =\sum_{I}\left(\omega_{I} \circ F\right) d\left(x^{i_{1}} \circ F\right) \wedge \cdots \wedge d\left(x^{i_{k}} \circ F\right) \\
& =\sum_{I}\left(\omega_{I} \circ F\right) d F^{i_{1}} \wedge \cdots \wedge d F^{i_{k}}
\end{aligned}
$$


where $F^{i_{j}}:=x^{i_{j}} \circ F$. Consequently,

$$
d F^{*} \omega=d_{V} F^{*} \omega=\sum_{I} d\left(\omega_{I} \circ F\right) \wedge d F^{i_{1}} \wedge \cdots d F^{i_{k}}
$$

where $d\left(\omega_{I} \circ F\right)$ is the differential of $\omega_{I} \circ F$. On the other hand,

$$
\begin{aligned}
F^{*} d \omega=F^{*}\left(d_{U} \omega\right) & =F^{*}\left(\sum_{I} d \omega_{I} \wedge d x^{i_{1}} \wedge \cdots \wedge d x^{i_{k}}\right) \\
& =\sum_{I} F^{*} d \omega_{I} \wedge F^{*} d x^{i_{1}} \wedge \cdots \wedge F^{*} d x^{i_{k}} \\
& =\sum_{I} d\left(\omega_{I} \circ F\right) d F^{i_{1}} \wedge \cdots \wedge d F^{i_{k}} .
\end{aligned}
$$

As in Corollary 19.6 ([30], p. 215), we obtain its abstract-definable version from Proposition 7.11 .

Corollary 7.12. Let $U$ be an abstract-definable open subset of $M$, and $\omega \in \Omega^{k}(M)$. Then, $\left.(d \omega)\right|_{U}=d\left(\left.\omega\right|_{U}\right)$.

Proof. As in Proposition 7.11, take $N$ as $U$, and $F$ as the inclusion map $\iota: U \rightarrow M$. Thus, we obtain

$$
d\left(\left.\omega\right|_{U}\right)=d(\omega \circ \iota)=d\left(\iota^{*} \omega\right)=\iota^{*} d \omega=(d \omega) \circ \iota=\left.(d \omega)\right|_{U}
$$





\section{Chapter 8}

\section{O-minimal de Rham cohomology}

In this chapter, we specify the o-minimal de Rham cohomology groups out of the cochain complexes of abstract-definable forms whose coboundary operators are the exterior derivatives constructed in the previous chapter, by following Chapter 7 of [30]. The computation of the 0th o-minimal de Rham cohomology group of an abstract-definable manifold gives a cartesian product of $\mathbb{R}$ as a result, since abstract-definable manifolds have only finitely many definably connected components, whereas the $k$ th o-minimal de Rham cohomology groups of $m$-dimensional abstract-definable manifolds are trivial for $k>m$, as in the classical case. The o-minimal de Rham cohomology has long exact sequences induced by short exact sequences of complexes of $\Omega^{k}(\cdot)$, the Mayer-Vietoris sequences, and also is invariant under abstract-definable diffeomorphisms. However, working whithin the context of an o-minimal expansion of the real field that possesses smooth cell decomposition and defines the exponential function is not enough for the invariance of the o-minimal de Rham cohomology under abstract-definable homotopy. The point is that there exist structures of such kind that do not define sufficiently many primitives. We then show that if we take the Pfaffian closure of this previously fixed structure as our framework and take a condition related to Bröcker's problem for granted, the abstract-definable hotomopy invariance is then obtained.

Recall, as fixed in Chapter 7 , that $\mathcal{R}$ denotes an o-minimal expansion of the real field $\mathbb{R}$ which admits smooth cell decomposition and defines the exponential function.

Throughout this chapter, unless otherwise stated, $(M, \mathcal{A})$ and $(N, \mathcal{B})$ denote abstractdefinable $\mathcal{C}^{\infty}$ manifolds of dimensions $m$ and $n$ respectively. 


\subsection{O-minimal de Rham cohomology}

An abstract-definable $\mathcal{C}^{\infty} k$-form $\omega$ on $M$ is said to be closed if its derivative vanishes, that is, $d \omega=0$, and exact if there is an abstract-definable $\mathcal{C}^{\infty}(k-1)$ form $\tau$ on $M$ such that $\omega=d \tau$. Observe that every exact abstract-definable $\mathcal{C}^{\infty}$ $k$-form on $M$ is closed, since $d^{2}=0$. We denote by $Z^{k}(M)$ the $\mathbb{R}$-vector space of all closed abstract-definable $\mathcal{C}^{\infty} k$-forms on $M$, and by $B^{k}(M)$ the $\mathbb{R}$-vector space of all exact abstract-definable $\mathcal{C}^{\infty} k$-forms on $M$. Hence, $B^{k}(M)$ is a subspace of $Z^{k}(M)$ and we may form the quotient vector space $Z^{k}(M) / B^{k}(M)$.

Definition 8.1. The $\mathbb{R}$-vector space

$$
H^{k}(M):=Z^{k}(M) / B^{k}(M)
$$

is called the $k$ th o-minimal de Rham cohomology group of $M$.

We put $H^{0}(M):=Z^{0}(M)$, since there is no exact abstract-definable $\mathcal{C}^{\infty} 0$-form on $M$. Moreover, because $\Omega^{k}(M)=0$ for all $k>m$, then $Z^{m}(M)=\Omega^{m}(M)$. The elements of $H^{k}(M)$, called cohomology classes, are $\approx$-equivalence classes $[\omega]$, where $\approx$ is an equivalence relation on $Z^{k}(M)$ defined as follows: for $\omega$ and $\tau$ in $Z^{k}(M)$

$$
\omega \approx \tau \stackrel{\text { def }}{\Leftrightarrow} \omega-\tau \in B^{k}(M) \Leftrightarrow \omega-\tau=d \eta \text {, for some } \eta \in \Omega^{k-1}(M) .
$$

When two closed abstract-definable $\mathcal{C}^{\infty} k$-forms in $\Omega^{k}(M)$ are $\approx$-equivalent, we say that they are cohomologous. Note that the $k$ th de Rham cohomology group $H^{k}(M)$ is not the trivial one $B^{k}(M)$ if and only if there exists closed abstractdefinable $\mathcal{C}^{\infty} k$-form on $M$ which fails to be exact.

Recall from Proposition 2.26 that $M$ has finitely many definably connected components.

Proposition 8.2. Let $j$ be the number of definably connected components of $M$. Then, the 0 th de Rham cohomology group of $M$ is $\mathbb{R}^{j}$, that is, $H^{0}(M) \cong \mathbb{R}^{j}$.

Proof. Let $D_{1}, \ldots, D_{j}$ denote the definably connected components of $M$. Observe that an abstract-definable function $f$ on $M$ is locally constant if and only if $f$ is constant on each $D_{i}$ (see Proposition 2.28). Furthermore, the vector space 
of the abstract-definable functions on $M$ which are constant on each definably connected component of $M$ is $j$-dimensional, since the abstract-definable functions $f_{i}: M \rightarrow \mathbb{R}$ given by

$$
f_{i}(x):= \begin{cases}1, & \text { if } x \in D_{i} \\ 0, & \text { if } x \notin D_{i}\end{cases}
$$

form a basis for it. So, if we show that the vector space $Z^{0}(M)$ agrees with the one of all locally constant abstract-definable functions on $M$, the proof is done. Fix an arbitrary chart $(U, \phi)=\left(U, x^{1}, \ldots, x^{m}\right)$ on $M$, and consider a closed abstractdefinable $\mathcal{C}^{\infty} 0$-form $f$ on $M$. We claim that $\left.f\right|_{U}$ is locally constant (hence so is $f$, for $U$ is open in $M)$. Since on $U$

$$
0=d f=\sum_{i=1}^{m} \frac{\partial f}{\partial x^{i}} d x^{i},
$$

the partial derivatives $\partial f / \partial x^{i}$ vanish on $U$. This is the same as asserting that the differential $d_{\phi(x)}\left(f \circ \phi^{-1}\right): \mathbb{R}^{m} \rightarrow \mathbb{R}$ of the definable $\mathcal{C}^{\infty}$ function $f \circ \phi^{-1}: \phi(U) \rightarrow \mathbb{R}$ is identically zero, for each $x \in U$. By Lemma $2.8([5], \text { p. 111 })^{1}$, it follows that $f \circ \phi^{-1}$ is constant on each definably connected component of $\phi(U)$. Because $\phi$ is a homeomorphism, $U$ is partitioned into finitely many open sets in $U$ so that $f$ is constant on each member of such a partition. Conversely, if $f$ is an abstractdefinable function which is locally constant on $M$, then so is on $U$. Let $x$ be any point in $U$, and let $U_{x}$ be an open neighborhood of $x$ in $U$ in which $f$ is constant. The definable function $f \circ \phi^{-1}$ is thus constant on the definable open set $\phi\left(U_{x}\right) \subseteq \mathbb{R}^{m}$, and as consequence, the following holds on $\phi\left(U_{x}\right)$

$$
0=\frac{\partial\left(f \circ \phi^{-1}\right)}{\partial r^{i}}=\frac{\partial f}{\partial x^{i}}
$$

for each $i \in\{1, \ldots, m\}$, resulting in the vanishing of $\left.d f\right|_{U_{x}}$. Therefore, $d f=0$ on $U$. Since the taken chart $(U, \phi)$ was arbitrary, the differential of $f$ is the identically zero map of $\Omega^{1}(M)$.

The following proposition is an immediate consequence of the fact that $\Omega^{k}(M)=0$ for $k>m$.

Proposition 8.3. For every $k>m, H^{k}(M) \cong 0$.

\footnotetext{
${ }^{1}$ Lemma 2.8. Let $f: U \rightarrow R^{n}$ be a definable $\mathcal{C}^{1}$ map, with $U \subseteq R^{m}$ definable open, and let $[a, b]:=\{(1-t) a+t b: 0 \leq t \leq 1\}$ be a line segment contained in $U$. Then $|f(b)-f(a)| \leq$ $|b-a| \max _{y \in[a, b]}\left|d_{y} f\right|$.
} 
Let $F: N \rightarrow M$ be an abstract-definable $\mathcal{C}^{k}$ map. As we have seen in the preceding chapter, such a map induces a homomorphism $F^{*}: \Omega^{*}(M) \rightarrow \Omega^{*}(N)$ of graded algebras, which we called the pullback map. In the sequel we shall see that the homomorphism $F^{*}$ induces in turn a linear map between the corresponding ominimal de Rham cohomology groups.

Lemma 8.4. Let $F: N \rightarrow M$ be an abstract-definable $\mathcal{C}^{\infty}$ map, and let $F^{*}: \Omega^{*}(M) \rightarrow$ $\Omega^{*}(N)$ be the induced pullback map. Then, $F^{*}\left(Z^{k}(M)\right) \subseteq Z^{k}(N)$ and $F^{*}\left(B^{k}(M)\right) \subseteq$ $B^{k}(N)$.

Proof. Let $\omega \in Z^{k}(M)$. Then, recalling from Proposition 7.11 that $F^{*}$ commutes with the exterior derivative $d$, it follows $d\left(F^{*} \omega\right)=F^{*}(d \omega)=0$. Also, if $\omega=d \eta$ for some $\eta \in \Omega^{k-1}(M)$, then $F^{*} \omega=F^{*}(d \eta)=d\left(F^{*} \eta\right)$.

The pullback map $F^{*}$ induces a map $F^{\sharp}: H^{k}(M) \rightarrow H^{k}(N)$ by setting

$$
F^{\sharp}([\omega]):=\left[F^{*} \omega\right] .
$$

From Lemma 8.4, it follows that $F^{\sharp}$ is well defined, and the linearity of $F^{*}$ implies that of $F^{\sharp}$. Moreover, if $\operatorname{id}_{M}: M \rightarrow M$ is the identity map, then $\operatorname{id}_{M}^{\sharp}: H^{k}(M) \rightarrow$ $H^{k}(M)$ is the identity map on $H^{k}(M)$; and for any abstract-definable $\mathcal{C}^{\infty}$ maps $F: N \rightarrow M$ and $G: M \rightarrow P$, we have $(G \circ F)^{\sharp}=F^{\sharp} \circ G^{\sharp}$. In other words, $\sharp$ is a contravariant functor from the category of abstract-definable $\mathcal{C}^{\infty}$ manifolds and abstract-definable $\mathcal{C}^{\infty}$ maps to the category of vector spaces on $\mathbb{R}$. Therefore, the following holds.

Proposition 8.5. The induced map $F^{\sharp}: H^{k}(M) \rightarrow H^{k}(N)$ as defined in (8.2) is a linear isomorphism whenever $F: N \rightarrow M$ is an abstract-definable $\mathcal{C}^{\infty}$ diffeomorphism.

The vector spaces $H^{k}(M)$ can be made into algebras over $\mathbb{R}$ through the introduction of the wedge product on the cohomology classes. Namely, for $[\omega],[\tau] \in H^{k}(M)$ we define

$$
[\omega] \wedge[\tau]:=[\omega \wedge \tau]
$$

This operation is well defined by the fact that the wedge product $\omega \wedge \tau$ is a closed abstract-definable $\mathcal{C}^{\infty}$ form on $M$, and the cohomology class $[\omega \wedge \tau]$ is independent 
of the choice of the representative of $[\tau]$ as well as of $[\omega]$. Therefore, equipping the $\mathbb{R}$-vector space

$$
H^{*}(M):=\bigoplus_{k=0}^{m} H^{k}(M)
$$

with the wedge product as in $(8.3), H^{*}(M)$ becomes a graded algebra over $\mathbb{R}$. The anticommutativity of $H^{*}(M)$ is inherited from that of $\Omega^{*}(M)$. If $F: N \rightarrow M$ is an abstract-definable $\mathcal{C}^{\infty}$ map, then the pullback map in cohomology $F^{\sharp}: H^{k}(M) \rightarrow$ $H^{k}(N)$ given as in (8.2) is a homomorphism of algebras, and thereby ${ }^{\sharp}$ is a contravariant functor from the category of abstract-definable $\mathcal{C}^{\infty}$ manifolds to the category of anticommutative graded algebras.

Let $V, W \subseteq M$ be abstract-definable open subsets covering $M$. Then, we have four inclusion maps (which are abstract-definable $\mathcal{C}^{\infty}$ maps) $: \imath_{V}: V \rightarrow M, \imath_{W}: W \rightarrow$ $M, \jmath_{V}: V \cap W \rightarrow V$, and $\jmath_{W}: V \cap W \rightarrow W$. Note that the pullback map $\imath_{V}^{*}: \Omega^{k}(M) \rightarrow \Omega^{k}(V)$ is the map that restricts the domain of an abstract-definable $\mathcal{C}^{\infty} k$-form on $M$ to $V$. The lemma below is the abstract-definable version of Proposition 26.2 ([30], p. 289) and its proof is virtually identical.

Lemma 8.6. Let $V, W \subseteq M$ be abstract-definable open cover of $M$. For each $k \geq 0$, the sequence below is exact

$$
0 \rightarrow \Omega^{k}(M) \stackrel{\iota_{k}}{\rightarrow} \Omega^{k}(V) \oplus \Omega^{k}(W) \stackrel{\jmath_{k}}{\rightarrow} \Omega^{k}(V \cap W) \rightarrow 0,
$$

where $\imath_{k}: \Omega^{k}(M) \rightarrow \Omega^{k}(V) \oplus \Omega^{k}(W)$ is the map given by

$$
\omega \mapsto\left(\imath_{V}^{*} \omega, \imath_{W}^{*} \omega\right)=\left(\left.\omega\right|_{V},\left.\omega\right|_{W}\right)
$$

whereas $\jmath_{k}: \Omega^{k}(V) \oplus \Omega^{k}(W) \rightarrow \Omega^{k}(V \cap W)$ is defined as

$$
(\omega, \tau) \mapsto \jmath_{V}^{*} \omega-\jmath_{W}^{*} \tau=\left.\omega\right|_{V \cap W}-\left.\tau\right|_{V \cap W}
$$

In the case $V \cap W$ is empty, we have $\Omega^{k}(V \cap W)=0$, and consequently $\jmath_{k}$ is the zero map.

Proof. We must prove the following statements, for each $k \geq 0$ : (i) $\imath_{k}$ is one-to-one; (ii) $\operatorname{Ker}\left(\jmath_{k}\right)=\operatorname{Im}\left(\iota_{k}\right)$; and (iii) $\jmath_{k}$ is onto.

(i) If $\imath_{k}(\omega)=(0,0)$, then $0=\imath_{V}^{*}(\omega)=\left.\omega\right|_{V}$ and $0=\imath_{W}^{*}(\omega)=\left.\omega\right|_{W}$, and since the sets $V, W$ cover $M$, it results that $\omega=0$. 
(ii) Let $(\omega, \tau) \in \Omega^{k}(V) \oplus \Omega^{k}(W)$ with $\jmath_{V}^{*} \omega-\jmath_{W}^{*} \tau=0$. This means that $\omega$ and $\tau$ agree on $V \cap W$. As a consequece, the map $\sigma: M \rightarrow \wedge^{k} T^{*} M$ given by

$$
\sigma:= \begin{cases}\omega, & \text { on } V \\ \tau, & \text { on } W\end{cases}
$$

is an abstract-definable $\mathcal{C}^{\infty} k$-form on $M$ satisfying $\imath_{k}(\sigma)=(\omega, \tau)$. Conversely, for any abstract-definable $\mathcal{C}^{\infty} k$-form $\omega$ on $M$, we have $\jmath_{k}\left(\imath_{k}(\omega)\right)=\jmath_{k}^{*}\left(\left.\omega\right|_{V},\left.\omega\right|_{W}\right)=$ $\left.\omega\right|_{V \cap W}-\left.\omega\right|_{V \cap W}=0$.

(iii) Let $\omega \in \Omega^{k}(V \cap W)$. By Proposition 7.1, there are abstract-definable $\mathcal{C}^{\infty}$ nonnegative functions $f_{V}, f_{W}: M \rightarrow R$ such that $f_{V}+f_{W}=1, \operatorname{supp}\left(f_{V}\right) \subseteq V$, and $\operatorname{supp}\left(f_{W}\right) \subseteq W$. Take $\sigma_{1} \in \Omega^{k}(V)$ and $\sigma_{2} \in \Omega^{k}(W)$ to be, respectively, the maps

$$
\sigma_{1}:= \begin{cases}f_{W} \cdot \omega, & \text { on } V \cap W \\ 0, & \text { on } V \backslash(V \cap W)\end{cases}
$$

and

$$
\sigma_{2}:=\left\{\begin{array}{ll}
-f_{V} \cdot \omega, & \text { on } \quad V \cap W \\
0, & \text { on } W \backslash(V \cap W)
\end{array} .\right.
$$

Hence,

$$
\jmath_{k}\left(\sigma_{1}, \sigma_{2}\right)=\jmath_{V}^{*} \sigma_{1}-\jmath_{W}^{*} \sigma_{2}=\left.\sigma_{1}\right|_{V \cap W}-\left.\sigma_{2}\right|_{V \cap W}=f_{W} \omega+f_{V} \omega=\left(f_{V}+f_{W}\right) \omega=\omega .
$$

Lemma 8.7. Let $V, W \subseteq M$ be abstract-definable open cover of $M$. For each $k \geq 0$, the diagram

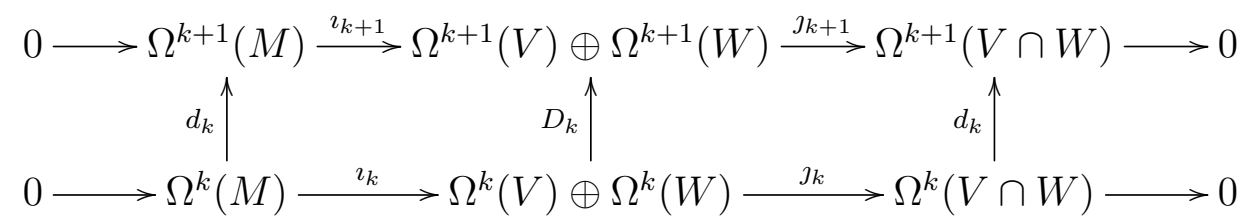

is commutative, where $D_{k}: \Omega^{k}(V) \oplus \Omega^{k}(W) \rightarrow \Omega^{k+1}(V) \oplus \Omega^{k+1}(W)$ is given by $D_{k}(\omega, \tau):=\left(d_{k} \omega, d_{k} \tau\right)$ and $\iota_{k}, \jmath_{k}$ are defined in Lemma 8.6. 
Proof. For any $\omega \in \Omega^{k}(M)$ and each $(\omega, \tau) \in \Omega^{k}(V) \oplus \Omega^{k}(W)$, by Corollary 7.12 we get

$D_{k}\left(\imath_{k} \omega\right)=D_{k}\left(\left.\omega\right|_{V},\left.\omega\right|_{W}\right)=\left(d_{k}\left(\left.\omega\right|_{V}\right), d_{k}\left(\left.\omega\right|_{W}\right)\right)=\left(\left.\left(d_{k} \omega\right)\right|_{V},\left.\left(d_{k} \omega\right)\right|_{W}\right)=\imath_{k+1}\left(d_{k} \omega\right)$,

and

$$
\begin{aligned}
d_{k}\left(j_{k}(\omega, \tau)\right)=d_{k}\left(\left.\omega\right|_{V \cap W}-\left.\tau\right|_{V \cap W}\right)=\left.\left(d_{k} \omega\right)\right|_{V \cap W}-\left.\left(d_{k} \tau\right)\right|_{V \cap W} & =j_{k+1}\left(d_{k} \omega, d_{k} \tau\right) \\
& =j_{k+1}\left(D_{k}(\omega, \tau)\right)
\end{aligned}
$$

Lemmas 8.6 and 8.7 imply that

$$
0 \rightarrow \Omega^{*}(M) \rightarrow \Omega^{*}(V) \oplus \Omega^{*}(W) \rightarrow \Omega^{*}(V \cap W) \rightarrow 0
$$

is a short exact sequence of cochain complexes. Hence, by the Zig-zag lemma (see Appendix B) and the fact that

$$
H^{k}(V) \oplus H^{k}(W) \simeq \frac{\operatorname{Ker}\left(D_{k}: \Omega^{k}(V) \oplus \Omega^{k}(W) \rightarrow \Omega^{k+1}(V) \oplus \Omega^{k+1}(W)\right)}{\operatorname{Im}\left(D_{k-1}: \Omega^{k-1}(V) \oplus \Omega^{k-1}(W) \rightarrow \Omega^{k}(V) \oplus \Omega^{k}(W)\right)}
$$

we obtain the following.

Theorem 8.8 (Mayer-Vietoris). Let $V, W \subseteq M$ be abstract-definable open sets covering $M$. There exists a long exact sequence

$$
\cdots \rightarrow H^{k-1}(V \cap W) \stackrel{\delta_{k-1}}{\rightarrow} H^{k}(M) \stackrel{\imath_{k}^{\sharp}}{\rightarrow} H^{k}(V) \oplus H^{k}(W) \stackrel{\jmath_{h}^{\sharp}}{\rightarrow} H^{k}(V \cap W) \rightarrow \cdots
$$

called the Mayer-Vietoris sequence, where $\imath_{k}^{\sharp}: H^{k}(M) \rightarrow H^{k}(V) \oplus H^{k}(W)$ is the linear $\operatorname{map} i_{k}^{\sharp}([\omega]):=\left(\left[\imath_{V}^{*} \omega\right],\left[\imath_{W}^{*} \omega\right]\right), j_{k}^{\sharp}: H^{k}(V) \oplus H^{k}(W) \rightarrow H^{k}(V \cap W)$ is given by $j_{k}^{\sharp}([\omega],[\tau]):=\left[j_{V}^{*} \omega-j_{W}^{*} \tau\right]$, and $\delta_{k}: H^{k}(V \cap W) \rightarrow H^{k+1}(M)$ is a connecting homomorphism (see Appendix B).

Observe that, since $\Omega^{k}(M)=0$ for $k<0$, the Mayer-Vietoris sequence as in the above theorem starts precisely with

$$
0 \rightarrow H^{0}(M) \rightarrow H^{0}(V) \oplus H^{0}(W) \rightarrow H^{0}(V \cap W) \rightarrow \cdots
$$




\subsection{Abstract-definable homotopy invariance}

This section is a rewriting of Section 27 ([30], pp. 296-301), concerning the notion of homotopy, in the context of abstract-definable manifolds.

Definition 8.9. Two abstract-definable $\mathcal{C}^{\infty}$ maps $F, G: M \rightarrow N$ are said to be definably homotopic, and denoted by $F \simeq G$, if there is an abstract-definable $\mathcal{C}^{\infty}$ map $H: M \times \mathbb{R} \rightarrow N$ such that

$$
H(x, 0)=F(x), \text { and } H(x, 1)=G(x) \text { for all } x \in M
$$

The map $H$ is called an abstract-definable $\mathcal{C}^{\infty}$ homotopy from $F$ to $G$.

Example 8.1 (Straight-line homotopy). Let $F, G: M \rightarrow \mathbb{R}^{n}$ be abstract-definable $\mathcal{C}^{\infty}$ maps. The natural definable homotopy $H: M \times \mathbb{R} \rightarrow \mathbb{R}^{n}$ defined as

$$
H(x, t):=(1-t) F(x)+t G(x)
$$

is called the straight-line homotopy from $F$ to $G$.

Definition 8.10. We say that an abstract-definable $\mathcal{C}^{\infty}$ map $F: M \rightarrow N$ is an abstract-definable $\mathcal{C}^{\infty}$ homotopy equivalence if there exists an abstractdefinable $\mathcal{C}^{\infty}$ map $G: N \rightarrow M$ such that $G \circ F \simeq \operatorname{id}_{M}$ and $F \circ G \simeq \operatorname{id}_{N}$. In this case, we also say that $M$ is definably homotopy equivalent to $N$, or that $M$ and $N$ have the same definable homotopy type. If $M$ has the definable homotopy type of a point, then $M$ is called definably contractible.

Example 8.2. $\mathbb{R}^{n}$ is definably contractible, since for any point $x$ in $\mathbb{R}^{n}$ the straightline homotopy gives a definable homotopy between the constant map $\imath \circ r: \mathbb{R}^{n} \rightarrow \mathbb{R}^{n}$, given by $y \mapsto x$, and the identity map $\operatorname{id}_{\mathbb{R}^{n}}$, where $\imath:\{x\} \rightarrow \mathbb{R}^{n}$ is the inclusion map and $r: \mathbb{R}^{m} \rightarrow\{x\}$ is the constant map.

Suppose $\mathfrak{R}$ is an o-minimal expansion of the real field $\mathbb{R}$, and $U$ is an $\mathfrak{R}$-definable open set subset of $\mathbb{R}^{n}$. Recall from $\left([13]\right.$, p. 2) that a $\mathcal{C}^{1}$ function $f: U \rightarrow \mathbb{R}$ is Pfaffian over $\mathfrak{R}$ if there exist $\mathfrak{R}$-definable $\mathcal{C}^{1}$ functions $P_{i}: U \times \mathbb{R} \rightarrow \mathbb{R}$ for $i=1, \ldots, n$ such that

$$
\frac{\partial f}{\partial r^{i}}(x)=P_{i}(x, f(x))
$$

for all $x \in U$. Now, let $\mathcal{L}(\mathfrak{R})$ be the collection of all (total) functions $f: \mathbb{R}^{n} \rightarrow \mathbb{R}$ for all $n \in \mathbb{N}$ that are Pfaffian over $\mathfrak{R}$. Set $\mathfrak{R}_{0}:=\mathfrak{R}$, and for each $i \geq 0$ let 
$\mathfrak{R}_{i+1}$ be the expansion of $\mathfrak{R}_{i}$ by all functions in $\mathcal{L}\left(\mathfrak{R}_{i}\right)$. Let $\mathcal{L}$ be the union of all $\mathcal{L}\left(\mathcal{R}_{i}\right)$ and let $\mathcal{P}(\mathfrak{R})$ be the expansion of $\mathfrak{R}$ by all the functions in $\mathcal{L}$. We call the structure $\mathcal{P}(\Re)$ the Pfaffian closure of $\mathfrak{R}$. Theorem 4.1 ([27], p. 209) together with Theorem 1 ([13], p. 2) ensures that the Pfaffian closure $\mathcal{P}(\mathfrak{R})$ of $\mathfrak{R}$ is o-minimal.

The discussion after Corollary 8.17 proves that working within the framework of an o-minimal expansion of the real which admits smooth cell decomposition and defines the exponential function is not sufficient for the validity of Theorem 8.14. This motivates the subsequent assumption.

From now on "definable" we mean "definable in $\mathcal{P}(\mathcal{R})$ with parameters in $\mathbb{R}$ ", where $\mathcal{P}(\mathcal{R})$ denotes the Pfaffian closure of $\mathcal{R}$, and by " $\mathcal{R}$-definable" we mean "definable in $\mathcal{R}$ with parameters in $\mathbb{R}$ ". Recall from [17] that $\mathcal{P}(\mathcal{R})$ admits smooth cell decomposition.

The following assertion, known as Bröcker's problem, was pointed out to us by P. Speissegger in an email exchange.

Claim 8.11 (Bröcker's problem). For any continuous function $b: \mathbb{R}^{n} \times \mathbb{R} \rightarrow \mathbb{R}$ which is definable in an o-minimal expansion $\mathfrak{R}$ of $\mathbb{R}$, the function $B: \mathbb{R}^{n} \rightarrow \mathbb{R}$, given by

$$
B(x):=\int_{0}^{1} b(x, t) d t,
$$

is definable in an o-minimal expansion $\widetilde{\mathfrak{R}}$ of $\mathfrak{R}$.

The above statement has been proved so far only for the case in which $\mathfrak{R}=\mathbb{R}_{\text {an }}$ and $\widetilde{\Re}=\mathbb{R}_{\text {an,exp }}$ by J.-M. Lion and J.-P. Rolin ([18]).

Lemma 8.12. Assume that the Bröcker's problem holds for any o-minimal expansion $\mathfrak{R}$ of $\mathbb{R}_{\exp }$ and for $\widetilde{\mathfrak{R}}$ taken to be the Pfaffian closure of $\mathfrak{R}$. If $b: \mathbb{R}^{n} \times \mathbb{R} \rightarrow \mathbb{R}$ is a definable $\mathcal{C}^{\infty}$ function, so is $B: \mathbb{R}^{n} \rightarrow \mathbb{R}$, where $B(x):=\int_{0}^{1} b(x, t) d t$. As a consequence, given an abstract-definable $\mathcal{C}^{\infty}$ function $b: N \times \mathbb{R} \rightarrow \mathbb{R}$, the function $\bar{b}: N \rightarrow \mathbb{R}$ given by $y \mapsto \int_{0}^{1} b(y, t) d t$ is abstract-definable $\mathcal{C}^{\infty}$ as well.

Proof. Since $b$ is definable in $\mathcal{P}(\mathcal{R})$, there is some $i \geq 0$ such that $b$ is definable in $\mathcal{R}_{i}$, where $\mathcal{R}_{0}:=\mathcal{R}$ (see the definition of Pfaffian closure above). Note, from the comments following Theorem 1 ([13], p. 2), that the Pfaffian closure of $\mathcal{R}$ can be obtained by adding only definable $\mathcal{C}^{\infty}$ total functions. In particular, $\mathcal{R}_{i}$ is 
an o-minimal expansion of $\mathbb{R}_{\exp }$ which admits smooth cell decomposition. Hence, the assumption implies that $B$ is definable in $\mathcal{P}\left(\mathcal{R}_{i}\right)$. The conclusion that $B$ is definable in $\mathcal{P}(\mathcal{R})$ follows from the fact that $\mathcal{P}\left(\mathcal{R}_{i}\right)$ and $\mathcal{P}(\mathcal{R})$ are interdefinable. The smoothness of $B$ is ensured, for instance, by Theorem C.14 ([15], p. 648).

Now, observe that for any chart $(V, \psi)$ on $N$ the composition $\bar{b} \circ \psi^{-1}: \mathbb{R}^{n} \rightarrow \mathbb{R}$ agrees with

$$
\psi(z) \mapsto \int_{0}^{1}\left(b \circ\left(\psi \times \mathrm{id}_{\mathbb{R}}\right)^{-1}\right)(\psi(z), t) d t .
$$

(Here we assumed the codomains of the charts $\psi: V \rightarrow \psi(V)$ are the whole $\mathbb{R}^{n}$, see Remark 2.12.) By hypothesis, $b \circ\left(\psi \times \mathrm{id}_{\mathbb{R}}\right)^{-1}$ is a definable $\mathcal{C}^{\infty}$ function on $\mathbb{R}^{n} \times \mathbb{R}$, and from the first part of the lemma it follows that $\bar{b} \circ \psi^{-1}$ is also definable $\mathcal{C}^{\infty}$. This proves that $\bar{b}$ is an abstract-definable $\mathcal{C}^{\infty}$ function.

Lemma 8.13. Let $U$ be an abstract-definable open subset of $N$, and let $\omega \in \Omega^{k}(U)$ with

$$
\operatorname{supp}(\omega) \subseteq F \subseteq U
$$

where $F$ is an abstract-definable closed subset of $N$. Then, $\omega$ can be extended to an abstract-definable $\mathcal{C}^{\infty} k$-form $\widetilde{\omega}$ on $N$.

Proof. Let $\omega$ be an abstract-definable $\mathcal{C}^{\infty} k$-form on $U$, and suppose $F$ is an abstract-definable closed set with $\operatorname{supp}(\omega) \subseteq F \subseteq U$. By Proposition 7.2, there exists an abstract-definable $\mathcal{C}^{\infty}$ function $\rho: M \rightarrow \mathbb{R}$ such that $0 \leq \rho \leq 1,\left.\rho\right|_{F}=1$, and $\operatorname{supp}(\rho) \subseteq U$. Take $\widetilde{\omega}: N \rightarrow \bigwedge^{k} T^{*} N$ to be

$$
\widetilde{\omega}(x):=\left\{\begin{array}{ll}
\rho(x) \omega(x), & \text { if } x \in U \\
0, & \text { otherwise }
\end{array} .\right.
$$

Firstly, note that $\widetilde{\omega}$ is well-defined as an abstract-definable $\mathcal{C}^{\infty}$ map from $N$ to $\bigwedge^{k} T^{*} N$. Also, for any $x \in U$, if $x \in \operatorname{supp}(\omega) \subseteq F$ then $\widetilde{\omega}(x)=\rho(x) \omega(x)=\omega(x)$; and if $x \in U \backslash \operatorname{supp}(\omega)$ then $\omega(x)=0=\rho(x) \cdot 0=\rho(x) \cdot \omega(x)=\widetilde{\omega}(x)$. In other words, $\left.\widetilde{\omega}\right|_{U}=\omega$.

For the remainder of the section, we assume that the Bröcker's problem holds for any o-minimal expansion $\mathfrak{R}$ of $\mathbb{R}_{\exp }$ and for $\widetilde{\mathfrak{R}}$ taken to be the Pfaffian closure of $\mathfrak{R}$. 
The proof of the following is virtually same as that of Theorem 27.10 ([30], p. $300)$, which is given in full detail in Section 29 ([30], p. 311).

Theorem 8.14 (Abstract-definable homotopy invariance). Suppose $f, g: N \rightarrow$ $M$ are two abstract-definable $\mathcal{C}^{\infty}$ maps with $f \simeq g$. Then the induced maps in cohomology $f^{\sharp}, g^{\sharp}: H^{*}(M) \rightarrow H^{*}(N)$ agree with each other.

Proof. Let $f, g: N \rightarrow M$ be abstract-definable $\mathcal{C}^{\infty}$ maps such that $f \simeq g$. Then, there exists an abstract-definable $\mathcal{C}^{\infty}$ map $H: N \times \mathbb{R} \rightarrow M$ satisfying $H\left(\iota_{0}(x)\right)=$ $f(x)$ and $H\left(\iota_{1}(x)\right)=g(x)$, for all $x \in N$, where $\iota_{t}: N \rightarrow N \times \mathbb{R}$ is the abstractdefinable $\mathcal{C}^{\infty}$ map given by $x \mapsto(x, t)$, for each fixed $t \in \mathbb{R}$. Since ${ }^{\sharp}$ is a contravariant functor, $\iota_{0}^{\sharp} \circ H^{\sharp}=f^{\sharp}$ and $\iota_{1}^{\sharp} \circ H^{\sharp}=g^{\sharp}$. Hence, in order to prove the theorem it suffices to show that $\iota_{0}^{\sharp}$ and $\iota_{1}^{\sharp}$ are the same. From Proposition B.6 in Appendix B, if there is a cochain homotopy $K:=\left\{K_{k}: \Omega^{k}(N \times \mathbb{R}) \rightarrow \Omega^{k-1}(N)\right\}_{k}$ between the induced pullback maps $\imath_{1}^{*}:=\left\{\left(\imath_{1}^{*}\right)_{k}: \Omega^{k}(N \times \mathbb{R}) \rightarrow \Omega^{k}(N)\right\}_{k}$ and $\imath_{0}^{*}:=\left\{\left(\imath_{0}^{*}\right)_{k}: \Omega^{k}(N \times \mathbb{R}) \rightarrow \Omega^{k}(N)\right\}_{k}$, then the induced maps in cohomology $\imath_{0}^{\sharp}$ and $\imath_{1}^{\sharp}$ agree with each other. In the remainder of the proof, we thus focus on establishing linear maps $K_{k}: \Omega^{k}(N \times \mathbb{R}) \rightarrow \Omega^{k-1}(N)$ for $k \geq 0$, which satisfy the equality

$$
d_{k-1} \circ K_{k}+K_{k+1} \circ d_{k}=\left(\imath_{1}^{*}\right)_{k}-\left(\imath_{0}^{*}\right)_{k}
$$

where $d_{k}$ denotes the exterior derivative on $\Omega^{k}(N \times \mathbb{R})$ and, as an abuse of notation, on $\Omega^{k}(N)$ as well.

Claim 8.15. Every abstract-definable $\mathcal{C}^{\infty} k$-form on $N \times \mathbb{R}$ can be written as a finite sum of abstract-definable $\mathcal{C}^{\infty}$ forms of the types:

(I) $a \pi^{*} \eta$;

(II) $b d t \wedge \pi^{*} \tau$,

where $a, b$ are abstract-definable $\mathcal{C}^{\infty}$ functions on $N \times \mathbb{R}, \pi: N \times \mathbb{R} \rightarrow N$ is the projection onto the first factor, $\eta$ is an abstract-definable $\mathcal{C}^{\infty} k$-form on $N$, and $\tau$ is an abstract-definable $\mathcal{C}^{\infty}(k-1)$-form on $N$.

Proof of the Claim. Let $\mathcal{B}$ denote the collection $\left\{\psi_{j}: V_{j} \rightarrow \psi_{j}\left(V_{j}\right) \subseteq \mathbb{R}^{n}\right\}_{j \in J}$, and fix an abstract-definable $\mathcal{C}^{\infty} k$-form $\omega$ on $N \times \mathbb{R}$. Let $\left\{\rho_{j}\right\}_{j \in J}$ be an abstractdefinable $\mathcal{C}^{\infty}$ partition of unity subordinate to $\mathcal{B}$ (see Lemma 4.6, [11]). By Proposition 7.2 , there exists a finite family $\left\{g_{j}\right\}_{j \in J}$ of abstract-definable $\mathcal{C}^{\infty}$ functions on 
$N$ such that for each $j$ : $0 \leq g_{j} \leq 1,\left.g_{j}\right|_{\operatorname{supp}\left(\rho_{j}\right)}=1$, and $\operatorname{supp}\left(g_{j}\right) \subseteq V_{j}$. Note that $\left\{\pi^{-1}\left(V_{j}\right)=V_{j} \times \mathbb{R}\right\}_{j \in J}$ is an abstract-definable open cover of $N \times \mathbb{R}$. Also, $\left\{\pi^{*} \rho_{j}\right\}_{j \in J}$ is an absctract-definable $\mathcal{C}^{\infty}$ partition of unity subordinate to $\left\{\pi^{-1}\left(V_{j}\right)\right\}_{j \in J}$ in the following sense:

(1) each $\pi^{*} \rho_{j}: N \times \mathbb{R} \rightarrow \mathbb{R}$ is an abstract-definable $\mathcal{C}^{\infty}$ nonnegative function;

(2) $\operatorname{supp}\left(\pi^{*} \rho_{j}\right) \subseteq \pi^{-1}\left(V_{j}\right)$, for each $j \in J$;

(3) $\sum_{j \in J} \pi^{*} \rho_{j}=1$.

Indeed, (1) follows immediately from the definition of pullback of abstract-definable $\mathcal{C}^{\infty} 0$-forms, that is, $\pi^{*} \rho_{j}=\rho_{j} \circ \pi$. Now, observe that

$$
\pi\left(\left\{(x, r) \in N \times \mathbb{R}: \rho_{j}(x) \neq 0\right\}\right)=\left\{x \in N: \rho_{j}(x) \neq 0\right\} \subseteq \operatorname{supp}\left(\rho_{j}\right) \subseteq V_{j} .
$$

Consequently,

$$
\left\{(x, r) \in N \times \mathbb{R}: \rho_{j}(x) \neq 0\right\} \subseteq \pi^{-1}\left(\operatorname{supp}\left(\rho_{j}\right)\right) \subseteq \pi^{-1}\left(V_{j}\right) .
$$

Since $\pi^{-1}\left(\operatorname{supp}\left(\rho_{j}\right)\right)$ is closed in $N \times \mathbb{R}$,

$$
\operatorname{supp}\left(\pi^{*} \rho_{j}\right)=\operatorname{cl}_{N \times \mathbb{R}}\left(\left\{(x, r) \in N \times \mathbb{R}: \rho_{j}(x) \neq 0\right\}\right) \subseteq \pi^{-1}\left(\operatorname{supp}\left(\rho_{j}\right)\right) \subseteq \pi^{-1}\left(V_{j}\right) .
$$

Thus, (2) follows. Finally, because $\sum_{j \in J} \pi^{*} \rho_{j}(z, t)=\sum_{j \in J} \rho_{j}(\pi(z, t))=\sum_{j \in J} \rho_{j}(z)$ for all $(z, t) \in N \times \mathbb{R}$, the validity of (3) is thereby obtained.

By virtue of (1)-(3), we can write $\omega$ as

$$
\omega=\left(\sum_{j \in J} \pi^{*} \rho_{j}\right) \omega=\sum_{j \in J}\left(\pi^{*} \rho_{j}\right) \omega=\sum_{j \in J} \omega_{j},
$$

where $\omega_{j}:=\left(\pi^{*} \rho_{j}\right) \omega \in \Omega^{k}(N \times \mathbb{R})$. Note that

$$
\operatorname{supp}\left(\omega_{j}\right) \subseteq \operatorname{supp}\left(\pi^{*} \rho_{j}\right) \cap \operatorname{supp}(\omega) \subseteq \operatorname{supp}\left(\pi^{*} \rho_{j}\right) \subseteq \pi^{-1}\left(V_{j}\right)
$$

If we show that each $\omega_{j}$ can be written as a finite sum of type-(I) and type-(II) abstract-definable $\mathcal{C}^{\infty}$ forms, then we are done. 
Let $\left(V_{j}, \psi_{j}\right)=\left(V_{j}, y^{1}, \ldots, y^{n}\right)$ be a chart in $\mathcal{B}$. Since $\pi^{-1}\left(V_{j}\right)=V_{j} \times \mathbb{R}$, the collection $\left\{\left(\pi^{-1}\left(V_{j}\right), \pi^{*} y^{1}, \ldots, \pi^{*} y^{n}, t\right)\right\}_{j \in J}$ forms an abstract-definable $\mathcal{C}^{\infty}$ atlas on $N \times \mathbb{R}$ (see Example 2.5), where $t$ is the projection $(x, r) \mapsto r: N \times \mathbb{R} \rightarrow \mathbb{R}$ restricted to $\pi^{-1}\left(V_{j}\right)$. Thus, on $\pi^{-1}\left(V_{j}\right)$, the abstract-definable $\mathcal{C}^{\infty} k$-form $\omega_{j}$ can be written uniquely as

$$
\begin{aligned}
\omega_{j} & =\sum_{1 \leq i_{1}<\cdots<i_{k} \leq n} a_{i_{1} \cdots i_{k}} d\left(\pi^{*} y^{i_{1}}\right) \wedge \cdots \wedge d\left(\pi^{*} y^{i_{k}}\right) \\
& +\sum_{1 \leq l_{1}<\cdots<l_{k-1} \leq n} b_{l_{1} \cdots l_{k-1}} d t \wedge d\left(\pi^{*} y^{l_{1}}\right) \wedge \cdots \wedge d\left(\pi^{*} y^{l_{k-1}}\right) \\
& =\sum_{I} a_{I} \pi^{*} d y^{I}+\sum_{L} b_{L} d t \wedge \pi^{*} d y^{L}
\end{aligned}
$$

after a rearrangement of the terms, where $I$ and $L$ denotes respectively $i_{1}<\ldots<$ $i_{k}$ and $l_{1}<\ldots<l_{k-1}, d y^{I}:=d y^{i_{1}} \wedge \cdots \wedge d y^{i_{k}}, d y^{L}:=d y^{l_{1}} \wedge \cdots \wedge d y^{l_{k-1}}$, and $a_{I}$, $b_{L}$ are abstract-definable $\mathcal{C}^{\infty}$ functions on $\pi^{-1}\left(V_{j}\right)$. Once by (8.7) we have

$$
\begin{aligned}
\operatorname{supp}\left(a_{i_{1} \cdots i_{k}}\right), \operatorname{supp}\left(b_{l_{1} \cdots l_{k-1}}\right) & \subseteq \mathrm{cl}_{\pi^{-1}\left(V_{j}\right)}\left(\left\{(x, t) \in \pi^{-1}\left(V_{j}\right): \omega_{j}(x, t) \neq 0\right\}\right) \\
& \subseteq \operatorname{supp}\left(\omega_{j}\right) \subseteq \operatorname{supp}\left(\pi^{*} \rho_{j}\right) \subseteq \pi^{-1}\left(V_{j}\right),
\end{aligned}
$$

we may then use Lemma 8.13 (with $F$ taken to be $\operatorname{supp}\left(\pi^{*} \rho_{j}\right)$ ) to obtain abstractdefinable $\mathcal{C}^{\infty} 0$-forms $\widetilde{a}_{I}, \widetilde{b}_{L}: N \times \mathbb{R} \rightarrow \mathbb{R}$ which extend $a_{I}$ and $b_{L}$ by zero, respectively. Note that we cannot proceed similarly for the abstract-definable $\mathcal{C}^{\infty}$ forms $d y^{I}, d y^{L}$ by applying Lemma 8.13, since the (topological) closure of the subsets of their domains in which $d y^{I}$ and $d y^{L}$ do not vanish coincide with their domains $V_{j}$, and this is not a closed subset of $N$. Nevertheless, we may get around this problem through the multiplication of $\omega_{j}$ by $\pi^{*} g_{j}$. In fact, because $\pi^{*} g_{j}=1$ on $\operatorname{supp}\left(\pi^{*} \rho_{j}\right)$ and $\operatorname{supp}\left(\omega_{j}\right) \subseteq \operatorname{supp}\left(\pi^{*} \rho_{j}\right)$, the equality $\omega_{j}=\left(\pi^{*} g_{j}\right) \omega_{j}$ holds. Therefore, on $\pi^{-1}\left(V_{j}\right), \omega_{j}$ can be rewritten as

$$
\begin{aligned}
\omega_{j}=\left(\pi^{*} g_{j}\right) \omega_{j} & =\sum_{I} a_{I}\left(\pi^{*} g_{j}\right) \pi^{*} d y^{I}+\sum_{L} b_{L} d t \wedge\left(\pi^{*} g_{j}\right) \pi^{*} d y^{L} \\
& =\sum_{I} a_{I} \pi^{*}\left(g_{j} d y^{I}\right)+\sum_{L} b_{L} d t \wedge \pi^{*}\left(g_{j} d y^{L}\right)
\end{aligned}
$$

Once $\operatorname{supp}\left(\left.g_{j}\right|_{V_{j}}\right) \subseteq \operatorname{supp}\left(g_{j}\right) \subseteq V_{j}$, we obtain by Lemma 8.13 extensions $\eta_{j}$ and $\tau_{j}$ by zero of $g_{j} d y^{I}$ and $g_{j} d y^{L}$ to $N$, respectively.

Finally, observe that the support of $\omega_{j} \in \Omega^{k}(N \times \mathbb{R})$ is contained in $\pi^{-1}\left(V_{j}\right)$ as well as the supports of each abstract-definable $\mathcal{C}^{\infty}$ form among $a_{I}, b_{L}, \pi^{*}\left(g_{j} d y^{I}\right)$, 
and $d t \wedge \pi^{*}\left(g_{j} d y^{L}\right)$. Thus, $\omega_{j}$ equals the extension by zero of $\left.\omega_{j}\right|_{\pi^{-1}\left(V_{j}\right)}$ to $N \times \mathbb{R}$, which in turn equals the sum of the products of the extension by zero (to $N \times \mathbb{R}$ ) of each term in (8.8), in other words,

$$
\omega_{j}=\sum_{I} \widetilde{a}_{I} \pi^{*} \eta_{j}+\sum_{L} \widetilde{b}_{L} d t \wedge \pi^{*} \tau_{j}
$$

with $\widetilde{a}_{I}, \widetilde{b}_{L} \in \Omega^{0}(N \times \mathbb{R}), \eta_{j} \in \Omega^{k}(N)$, and $\tau_{j} \in \Omega^{k-1}(N)$.

Define $K_{k}: \Omega^{k}(N \times \mathbb{R}) \rightarrow \Omega^{k-1}(N)$ by:

(i) $K_{k}\left(a \pi^{*} \eta\right):=0$, on type-(I) abstract-definable $\mathcal{C}^{\infty} k$-forms;

(ii) $K_{k}\left(b d t \wedge \pi^{*} \tau\right):=\left(\int_{0}^{1} b(y, t) d t\right) \tau$, on type-(II) abstract-definable $\mathcal{C}^{\infty} k$-forms;

(iii) $K_{k}$ is extended linearly.

After fixing an abstract-definable $\mathcal{C}^{\infty}$ partition of unity $\left\{\rho_{j}\right\}_{j}$ subordinate to $\mathcal{B}$, and a finite collection $\left\{g_{j}\right\}_{j \in J}$ of abstract-definable $\mathcal{C}^{\infty}$ functions on $N$, we can express $\omega \in \Omega^{k}(N \times \mathbb{R})$ as a sum $\omega=\sum_{j} \omega_{j}$, where $\omega_{j}$ is decomposed uniquely into

$$
\sum_{j, I} a_{I}^{j} \pi^{*} \eta_{j}+\sum_{j, L} b_{L}^{j} d t \wedge \pi^{*} \tau_{j}
$$

like in (8.9) (see the proof of Claim 8.15). So, $K_{k}(\omega)=\sum_{j, L}\left(\int_{0}^{1} b_{L}^{j}(x, t) d t\right) \tau_{j}$. (Lemma 8.12 shows that each $\int_{0}^{1} b_{L}^{j}(x, t) d t$ is an abstract-definable $\mathcal{C}^{\infty}$ function on $N$, therefore $K_{k}(\omega)$ lies indeed in $\Omega^{k-1}(N)$.)

Let us now check (8.6). Fix a chart $\left(V \times \mathbb{R}, \pi^{*} y^{1}, \ldots, \pi^{*} y^{n}, t\right)$ on $N \times \mathbb{R}$. For type-(I) abstract-definable $\mathcal{C}^{\infty} k$-forms, we have

$$
\begin{aligned}
\left(K_{k+1} \circ d_{k}\right)\left(a \pi^{*} \eta\right) & =K_{k+1}\left(d_{0} a \wedge \pi^{*} \eta+a d_{k}\left(\pi^{*} \eta\right)\right) \\
& =K_{k+1}\left(\left(\sum_{i=1}^{n} \frac{\partial a}{\partial \pi^{*} y^{i}} d\left(\pi^{*} y^{i}\right)+\frac{\partial a}{\partial t} d t\right) \wedge \pi^{*} \eta+a \pi^{*}\left(d_{k} \eta\right)\right) \\
& =K_{k+1}\left(\frac{\partial a}{\partial t} d t \wedge \pi^{*} \eta+\sum_{i=1}^{n} \frac{\partial a}{\partial \pi^{*} y^{i}} \pi^{*}\left(d_{0} y^{i}\right) \wedge \pi^{*} \eta+a \pi^{*} d_{k} \eta\right) \\
& =K_{k+1}\left(\frac{\partial a}{\partial t} d t \wedge \pi^{*} \eta\right)+\sum_{i=1}^{n} K_{k+1}\left(\frac{\partial a}{\partial \pi^{*} y^{i}} \pi^{*}\left(d_{0} y^{i} \wedge \eta\right)\right)+K_{k+1}\left(a \pi^{*} d_{k} \eta\right) \\
& =\left(\int_{0}^{1} \frac{\partial a}{\partial t} d t\right) \eta \\
& =(a(x, 1)-a(x, 0)) \eta=\imath_{1}^{*} a\left(\imath_{1}^{*} \pi^{*} \eta\right)-\imath_{0}^{*} a\left(\imath_{0}^{*} \pi^{*} \eta\right)=\left(\imath_{1}^{*}-\imath_{0}^{*}\right)\left(a \pi^{*} \eta\right)
\end{aligned}
$$


and $\left(d_{k-1} \circ K_{k}\right)\left(a \pi^{*} \eta\right)=d_{k-1}(0)=0$. Hence, $\left(d_{k-1} \circ K_{k}+K_{k+1} \circ d_{k}\right)\left(a \pi^{*} \eta\right)=$ $\left(\imath_{1}^{*}-\imath_{0}^{*}\right)\left(a \pi^{*} \eta\right)$. For type-(II) abstract-definable $\mathcal{C}^{\infty} k$-forms, we get

$$
\begin{aligned}
\left(d_{k-1} \circ K_{k}\right)\left(b d t \wedge \pi^{*} \tau\right) & =d_{k-1}\left(\left(\int_{0}^{1} b(y, t) d t\right) \tau\right) \\
& =d_{k-1}\left(\int_{0}^{1} b(y, t) d t\right) \wedge \tau+\left(\int_{0}^{1} b(y, t) d t\right) d_{k-1} \tau \\
& =\sum_{i=1}^{n} \frac{\partial}{\partial y^{i}}\left(\int_{0}^{1} b(y, t) d t\right) d y^{i} \wedge \tau+\left(\int_{0}^{1} b(y, t) d t\right) d_{k-1} \tau
\end{aligned}
$$

and also

$$
\begin{aligned}
\left(K_{k+1} \circ d_{k}\right)\left(b d t \wedge \pi^{*} \tau\right) & =K_{k+1}\left(d_{1}(b d t) \wedge \pi^{*} \tau-b d t \wedge d_{k}\left(\pi^{*} \tau\right)\right) \\
& =K_{k+1}\left(d_{1} b \wedge d t \wedge \pi^{*} \tau\right)-K_{k+1}\left(b d t \wedge \pi^{*} d_{k-1} \tau\right) \\
& =K_{k+1}\left(\sum_{i=1}^{n} \frac{\partial b}{\partial \pi^{*} y^{i}} d \pi^{*} y^{i} \wedge d t \wedge \pi^{*} \tau\right)-K_{k+1}\left(b d t \wedge \pi^{*} d_{k-1} \tau\right) \\
& =-\sum_{i=1}^{n} K_{k+1}\left(\frac{\partial b}{\partial \pi^{*} y^{i}} d t \wedge \pi^{*}\left(d y^{i} \wedge \tau\right)\right)-\left(\int_{0}^{1} b(y, t) d t\right) d_{k-1} \tau \\
& =-\sum_{i=1}^{n}\left(\int_{0}^{1} \frac{\partial b}{\partial \pi^{*} y^{i}}(y, t) d t\right) d y^{i} \wedge \tau-\left(\int_{0}^{1} b(y, t) d t\right) d_{k-1} \tau \\
& =-\sum_{i=1}^{n} \frac{\partial}{\partial y^{i}}\left(\int_{0}^{1} b(y, t) d t\right) d y^{i} \wedge \tau-\left(\int_{0}^{1} b(y, t) d t\right) d_{k-1} \tau
\end{aligned}
$$

where the last equality followed from the differentiation under the integral sign. Furthermore, $\imath_{1}^{*}\left(b d t \wedge \pi^{*} \tau\right)=b(y, 1) \imath_{1}^{*}(d t) \wedge \imath_{1}^{*}\left(\pi^{*} \tau\right)=0$, since $\imath_{1}^{*}(d t)=d\left(\imath_{1}^{*} t\right)=$ $d(1)=0$. Similarly, $\imath_{0}^{*}\left(b d t \wedge \pi^{*} \tau\right)=0$. Therefore,

$$
\left(d_{k-1} \circ K_{k}+K_{k+1} \circ d_{k}\right)\left(b d t \wedge \pi^{*} \tau\right)=0=\left(\imath_{1}^{*}-\imath_{0}^{*}\right)\left(b d t \wedge \pi^{*} \tau\right) .
$$

This finishes the proof.

Corollary 8.16. If $M$ and $N$ are definably homotopy equivalent, then $H^{k}(M) \cong$ $H^{k}(N)$ for all $k \geq 0$.

Proof. By definition, there are abstract-definable $\mathcal{C}^{\infty}$ maps $f: M \rightarrow N$ and $g: N \rightarrow$ $M$ such that $f \circ g \simeq \operatorname{id}_{N}$ and $g \circ f \simeq \operatorname{id}_{M}$. By Theorem 8.14 and the fact that ${ }^{\sharp}$ is 
a contravariant functor,

$$
\operatorname{id}_{H^{*}(M)}=\left(\operatorname{id}_{M}\right)^{\sharp}=(g \circ f)^{\sharp}=f^{\sharp} \circ g^{\sharp}
$$

and

$$
\operatorname{id}_{H^{*}(N)}=\left(\operatorname{id}_{N}\right)^{\sharp}=(f \circ g)^{\sharp}=g^{\sharp} \circ f^{\sharp} .
$$

Therefore, the linear maps $f^{\sharp}, g^{\sharp}$ are inverses of each other. Particularly, $H^{k}(M) \cong$ $H^{k}(N)$ for each $k \geq 0$.

Corollary 8.17 (Poincaré lemma). If $M$ is definably contractible, then the ominimal de Rham cohomology of $M$ is

$$
H^{k}(M) \cong \begin{cases}\mathbb{R}, & \text { if } k=0 \\ 0, & \text { if } k>0\end{cases}
$$

Proof. From the definable contraction of $M$ and Corollary 8.16, it follows that there is some point $a$ in $M$ for which $H^{k}(M) \cong H^{k}(\{a\})$ for all $k \geq 0$. Recall from Example 2.2 that the abstract-definable $\mathcal{C}^{\infty}$ manifold $\{a\}$ has dimension 0 , and thereby $H^{k}(\{a\}) \cong 0$ for $k>0$, according to Proposition 8.3. Moreover, since $\{a\}$ is definably connected, $H^{0}(\{a\}) \cong \mathbb{R}$, by Proposition 8.2.

Corollary 8.17 picks o-minimal expansions of the real field as candidates for which Theorem 8.14 holds. These are to some extent large o-minimal expansions of the real field possessing smooth cell decomposition and defining the exponential function, i.e., those that define sufficiently many primitives. The real exponential field $\mathbb{R}_{\exp }:=\langle\mathbb{R},<, 0,1,+, \cdot, \exp \rangle$ is not one of these structures. Indeed, consider the closed definable (in $\mathbb{R}_{\exp }$ ) $\mathcal{C}^{\infty} 1$-form

$$
\frac{-y}{x^{2}+y^{2}} d x+\frac{x}{x^{2}+y^{2}} d y
$$

on $X:=\mathbb{R}^{2} \backslash\{(x, y): x \geq 0, y=0\}$. From elementary calculus we know that $\tau+\pi$, where

$$
\tau(x, y):= \begin{cases}\frac{\pi}{2}-\arctan \left(\frac{x}{y}\right) & \text { if } y>0 \\ \pi & \text { if } y=0 \text { and } x<0 \\ \frac{3 \pi}{2}-\arctan \left(\frac{x}{y}\right) & \text { if } y<0\end{cases}
$$


is the primitive of the abstract-definable form in question, and by trigonometric identities and Theorem 1 ([2], p. 1174) it follows that $\tau+\pi$ is not definable in $\mathbb{R}_{\text {exp. }}$. In other words, $H^{1}(X) \neq 0$. Since $H_{\mathrm{dR}}^{1}(X)=0$ (the 1 st de Rham cohomology group of $X$ ), we conclude that the classical and the o-minimal de Rham cohomologies do not coincide. 



\section{Appendix A}

\section{Multilinear algebra}

\section{A.1 Wedge product}

Throughout this appendix $V$ denotes an $m$-dimensional vector space on a field $\mathbb{K}$, unless otherwise stated.

A $k$-linear function $f: \overbrace{V \times \cdots \times V}^{k \text { times }} \rightarrow \mathbb{K}$, with $k \in \mathbb{N}$, is called a $k$-tensor on $V$. A $k$-tensor is said to be alternating (or a $k$-covector) if for any permutation $\sigma \in \mathbb{S}_{k}$

$$
f\left(v_{\sigma(1)}, \ldots, v_{\sigma(k)}\right)=(\operatorname{sgn} \sigma) f\left(v_{1}, \ldots, v_{k}\right) .
$$

We denote by $\bigwedge^{k} V^{*}$ the $\mathbb{K}$-vector space of the alternating $k$-tensors on $V$. Observe that $\bigwedge^{0} V^{*} \cong \mathbb{K}$ and $\bigwedge^{1} V^{*}=V^{*}$.

Remark A.1. An important observation is that an alternating $k$-tensor $f$ on $V$ is completely determined by its values at the $k$-tuples $\left(e_{i_{1}}, \ldots, e_{i_{k}}\right)$, where $1 \leq i_{1}<$ $\cdots<i_{k} \leq m$ and $\left\{e_{1}, \ldots, e_{m}\right\}$ is a basis for $V$.

If $f$ and $g$ are alternating $k$ - and $l$-tensors on $V$, respectively, their wedge product (or exterior product) is the alternating $(k+l)$-tensor on $V$ defined by

$$
(f \wedge g)\left(v_{1}, \ldots, v_{k+l}\right):=\frac{1}{k ! l !} \sum_{\sigma \in \mathbb{S}_{k+l}}(\operatorname{sgn} \sigma) f\left(v_{\sigma(1)}, \ldots, v_{\sigma(k)}\right) g\left(v_{\sigma(k+1)}, \ldots, v_{\sigma(k+l)}\right)
$$

If $f \in \bigwedge^{0} V^{*}$, that is, $f$ is an element of $\mathbb{K}$, then $f \wedge g=f g$ for any $g \in \bigwedge^{l} V^{*}$.

Proposition A.2 ([30], pp. 27-29). Let $f \in \bigwedge^{k} V^{*}, g \in \bigwedge^{l} V^{*}, h \in \bigwedge^{n} V^{*}$, and $c \in \mathbb{K}$. Then, we have the following properties. 
(1) (Associativity) $f \wedge(g \wedge h)=(f \wedge g) \wedge h$.

(2) (Bilinearity) $f \wedge(g+c h)=f \wedge g+c(f \wedge h)$ and $(f+c g) \wedge h=f \wedge h+c(g \wedge h)$.

(3) (Anticommutativity) $f \wedge g=(-1)^{k l} g \wedge f$.

An immediate consequence of (3) in the above proposition is $f \wedge f=0$, for any $f \in \bigwedge^{k} V^{*}$ where $k$ is odd.

Proposition A.3 ([30], p. 30). If $\alpha^{1}, \ldots, \alpha^{k}$ are linear functions on $V$, with $k \leq m$, then

$$
\left(\alpha^{1} \wedge \cdots \wedge \alpha^{k}\right)\left(v_{1}, \ldots, v_{k}\right)=\operatorname{det}\left[\begin{array}{ccc}
\alpha^{1}\left(v_{1}\right) & \cdots & \alpha^{1}\left(v_{k}\right) \\
\vdots & \ddots & \vdots \\
\alpha^{k}\left(v_{1}\right) & \cdots & \alpha^{k}\left(v_{k}\right)
\end{array}\right]
$$

Notation. Let $\left\{e_{1}, \ldots, e_{m}\right\}$ be a basis for $V$ and $\left\{\epsilon^{1}, \ldots, \epsilon^{m}\right\}$ its dual basis for $V^{*}$. Given a multi-index $I:=\left(i_{1}, \ldots, i_{k}\right)$ with $i_{j} \in\{1, \ldots, m\}$, we write $e_{I}$ for $\left(e_{i_{1}}, \ldots, e_{i_{k}}\right)$ and $\epsilon^{I}$ for $\epsilon^{i_{1}} \wedge \cdots \wedge \epsilon^{i_{k}}$.

Lemma A.4 ([30], p. 31). With the same notation as above, if $I:=\left(1 \leq i_{1}<\right.$ $\left.\cdots<i_{k} \leq m\right)$ and $J:=\left(1 \leq j_{1}<\cdots<j_{k} \leq m\right)$, then

$$
\epsilon^{I}\left(e_{J}\right)=\delta_{J}^{I}=\left\{\begin{array}{ll}
1, & \text { if } I=J \\
0, & \text { if } I \neq J
\end{array} .\right.
$$

Proposition A.5 ([30], pp. 31-32). Let $\left\{e_{1}, \ldots, e_{m}\right\}$ be a basis for $V$ and let $\left\{\epsilon^{1}, \ldots, \epsilon^{m}\right\}$ be its dual basis for $V^{*}$. Then,

$$
\left\{\epsilon^{I} \mid I:=\left(1 \leq i_{1}<\cdots<i_{k} \leq m\right)\right\}
$$

forms a basis for $\bigwedge^{k} V^{*}$. In particular, $\operatorname{dim}\left(\bigwedge^{k} V^{*}\right)=\left(\begin{array}{c}m \\ k\end{array}\right)$.

Corollary A.6 ([30], p. 32). If $k>m$, then $\bigwedge^{k} V^{*}=0$. 


\section{A.2 Graded algebra}

An algebra $A$ over a field $\mathbb{K}$ is said to be graded if it can be written as a direct sum

$$
A=\bigoplus_{k=0}^{\infty} A^{k}
$$

of $\mathbb{K}$-vector spaces such that the multiplication map on $A$ sends $A^{k} \times A^{l}$ to $A^{k+l}$. The notation $A=\bigoplus_{k=0}^{\infty} A^{k}$ means that each nonzero element $a$ of $A$ is uniquely expressed as a finite sum

$$
a=a_{i_{1}}+\cdots+a_{i_{l}}
$$

with $0 \neq a_{i_{j}} \in A^{i_{j}}$. A graded algebra $A$ is called anticommutative (or graded commutative) if for all $a \in A^{k}$ and $b \in A^{l}$,

$$
a b=(-1)^{k l} b a
$$

A homomorphism of graded algebras is an algebra homomorphism that preserves the degree (which is $k$ for $a \in A^{k}$ ).

Example A.1. Let $V$ be a $\mathbb{K}$-vector space, with $\operatorname{dim} V=m \in \mathbb{N}$, the $\mathbb{K}$-vector space

$$
\bigwedge^{\bullet} V^{*}:=\bigoplus_{k=0}^{\infty} \bigwedge^{k} V^{*}=\bigoplus_{k=0}^{m} \bigwedge^{k} V^{*}
$$

with the wedge product of multicovectors as multiplication makes $\wedge^{\bullet} V^{*}$ into an anticommutative graded algebra, called the exterior algebra (or the Grassmann algebra) of multicovectors on $V$.

An antiderivation on a graded algebra $A:=\bigoplus_{k=0}^{\infty} A^{k}$ is a $\mathbb{K}$-linear map $D: A \rightarrow$ $A$ such that

$$
D(\omega \cdot \tau)=(D \omega) \cdot \tau+(-1)^{k} \omega \cdot D \tau
$$

for $\omega \in A^{k}$ and $\tau \in A^{l}$. In the graded algebra $A$, an element of $A^{k}$ is called a homogeneous element of degree $k$. The antiderivation is of degree $m$ if

$$
\operatorname{deg}(D \omega)=\operatorname{deg}(\omega)+m
$$

for all homogeneous elements $\omega \in A$. 



\section{Appendix B}

\section{Homological algebra}

\section{B.1 Cochain complexes}

Definition B.1 ([30], p. 281). A cochain complex $\mathcal{C}$ is a collection of vector spaces $\left\{C^{k}\right\}_{k \in \mathbb{Z}}$ together with a sequence of linear maps $d_{k}: C^{k} \rightarrow C^{k+1}$ such that $d_{k+1} \circ d_{k}=0$ for each $k$ in $\mathbb{Z}$. Pictorially, $\mathcal{C}$ is the diagram

$$
\cdots \stackrel{d_{-1}}{\rightarrow} C^{0} \stackrel{d_{0}}{\rightarrow} C^{1} \stackrel{d^{1}}{\rightarrow} C^{2} \stackrel{d_{2}}{\rightarrow} \ldots
$$

The collection $\left\{d_{k}\right\}_{k \in \mathbb{Z}}$ of linear maps is called the differential of the cochain complex $\mathcal{C}$.

Definition B.2 ([30], p. 281). A sequence of vector spaces

$$
A \stackrel{f}{\rightarrow} B \stackrel{g}{\rightarrow} C
$$

is said to be exact at $B$ whenever $\operatorname{Im}(f)=\operatorname{Ker}(g)$. A $k$-term sequence

$$
C^{0} \stackrel{d_{0}}{\rightarrow} C^{1} \stackrel{d^{1}}{\rightarrow} C^{2} \stackrel{d_{2}}{\rightarrow} \ldots \stackrel{d_{k-1}}{\rightarrow} C^{k}
$$

of vector spaces, which is exact at every term except the first and the last, is simply called an exact sequence. A five-term exact sequence of the form

$$
0 \rightarrow A \rightarrow B \rightarrow C \rightarrow 0
$$

is said to be a short exact sequence. 
Note that if $A=0$, then the sequence

$$
A \stackrel{f}{\rightarrow} B \stackrel{g}{\rightarrow} C
$$

is exact if and only if $g$ is injective; and if $C=0$, such a sequence is exact if and only if $f$ is surjective.

The following propositions are straightforward.

Proposition B.3 ([30], p. 282). If $A \stackrel{f}{\rightarrow} B \stackrel{g}{\rightarrow} C$ is an exact sequence, then

(1) the map $f$ is surjective if and only if $g$ is the zero map;

(2) the map $g$ is injective if and only if $f$ is the zero map.

Proposition B.4 ([30], p. 282).

(1) the four-term sequence $0 \rightarrow A \stackrel{f}{\rightarrow} B \rightarrow 0$ of vector spaces is exact if and only if $f: A \rightarrow B$ is a linear isomorphism;

(2) if $A \stackrel{f}{\rightarrow} B \rightarrow C \rightarrow 0$ is an exact sequence of vector spaces, then there is a linear isomorphism

$$
C \simeq \operatorname{Coker}(f):=B / \operatorname{Im}(f)
$$

If $\mathcal{C}$ is a cochain complex, then from $d_{k} \circ d_{k-1}=0$ it follows that $\operatorname{Im}\left(d_{k-1}\right) \subseteq$ $\operatorname{Ker}\left(d_{k}\right)$. We can therefore form the quotient vector space

$$
H^{k}(\mathcal{C}):=\frac{\operatorname{Ker}\left(d_{k}\right)}{\operatorname{Im}\left(d_{k-1}\right)},
$$

called the $k$ th cohomology vector space of the cochain complex $\mathcal{C}$. Such a vector space measures the extent to which the cochain complex $\mathcal{C}$ fails to be exact at $C^{n}$. We call $k$-cochains the elements of the vector space $C^{k}$. A $k$ cochain in $\operatorname{Ker}\left(d_{k}\right)$ is called a $k$-cocycle and a $k$-cochain in $\operatorname{Im}\left(d_{k-1}\right)$ is called a $k$-coboundary. The elements of $H^{k}(\mathcal{C})$ are called cohomology classes. We denote the subspaces of $k$-cocycles and $k$-coboundaries of $\mathcal{C}$ by $Z^{k}(\mathcal{C})$ and $B^{k}(\mathcal{C})$, respectively.

If $\mathcal{A}$ and $\mathcal{B}$ are two cochain complexes with differentials $d$ and $d^{\prime}$ respectively, a cochain map $\varphi: \mathcal{A} \rightarrow \mathcal{B}$ is a collection of linear maps $\left\{\varphi_{k}: A^{k} \rightarrow B^{k}\right\}_{k \in \mathbb{Z}}$ that commute with $d$ and $d^{\prime}$, that is, $d_{k}^{\prime} \circ \varphi_{k}=\varphi_{k+1} \circ d_{k}$ for each $k$. 
A cochain map $\varphi: \mathcal{A} \rightarrow \mathcal{B}$ induces linear maps in cohomology $\varphi^{\sharp}: H^{k}(\mathcal{A}) \rightarrow H^{k}(\mathcal{B})$ by

$$
\varphi_{k}^{\sharp}([a]):=\left[\varphi_{k}(a)\right], a \in Z^{k}(\mathcal{A}) .
$$

It is not hard to show that the induced map $\varphi_{k}^{\sharp}$ is well defined.

A sequence of cochain complexes

$$
0 \rightarrow \mathcal{A} \stackrel{i}{\rightarrow} \mathcal{B} \stackrel{j}{\rightarrow} \mathcal{C} \rightarrow 0
$$

is short exact if for each $k$

$$
0 \rightarrow A^{k} \stackrel{i_{k}}{\rightarrow} B^{k} \stackrel{j_{k}}{\rightarrow} C^{k} \rightarrow 0
$$

is a short exact sequence of vector spaces.

For a given short exact sequence $0 \rightarrow \mathcal{A} \stackrel{i}{\rightarrow} \mathcal{B} \stackrel{j}{\rightarrow} \mathcal{C} \rightarrow 0$ of cochain complexes, we can construct for each $k$ a linear map $\delta_{k}: H^{k}(\mathcal{C}) \rightarrow H^{k+1}(\mathcal{A})$, called the connecting homomorphism, as follows. Consider the short exact sequences for $k$ and $k+1$ :

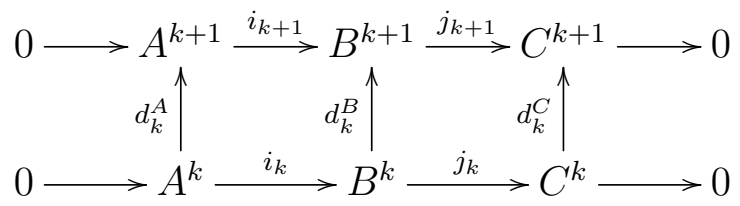

For any $[c] \in H^{k}(\mathcal{C})$, there exists $b \in B^{k}$ such that $j_{k}(b)=c$ since $j_{k}$ is surjective. By the commutativity of the right hand-side square in the diagram and the fact that $d_{k}^{C}(c)=0, d_{k}^{B}(b) \in \operatorname{Ker}\left(j_{k+1}\right)=\operatorname{Im}\left(i_{k+1}\right)$. Therefore, $d_{k}^{B}(b)=i_{k+1}(a)$ for some $a \in A^{k+1}$. Because $i_{k+2}\left(d_{k+1}^{A}(a)\right)=d_{k+1}^{B}\left(i_{k+1}(a)\right)=d_{k+1}^{B}\left(d_{k}^{B}(b)\right)=0$ and $i_{k+2}$ is injective, $d_{k+1}^{A}(a)=0$. Then, we set

$$
\delta_{k}[c]:=[a] \in H^{k+1}(\mathcal{A}) .
$$

A routine argument shows that $\delta_{k}$ is a well-defined linear map.

Theorem B.5 (The zig-zag lemma, [30], p. 285). A short exact sequence of cochain complexes

$$
0 \rightarrow \mathcal{A} \stackrel{i}{\rightarrow} \mathcal{B} \stackrel{j}{\rightarrow} \mathcal{C} \rightarrow 0
$$

gives rise to a long exact sequence in cohomology

$$
\cdots \rightarrow H^{k}(\mathcal{A}) \stackrel{i_{k}^{\sharp}}{\rightarrow} H^{k}(\mathcal{B}) \stackrel{j^{\sharp}}{\rightarrow} H^{k}(\mathcal{C}) \stackrel{\delta_{k}}{\rightarrow} H^{k+1}(\mathcal{A}) \rightarrow \cdots,
$$


where $i_{k}^{\sharp}$ and $j_{k}^{\sharp}$ are the maps in cohomology induced by the cochain maps $i$ and $j$, and $\delta_{k}$ are the connecting homomorphisms.

\section{B.2 Cochain homotopy}

Let $\varphi, \psi: \mathcal{A} \rightarrow \mathcal{B}$ be two cochain maps. A cochain homotopy $K$ from $\varphi$ to $\psi$ is a collection of homomorphisms $\left\{K_{k}: A^{k} \rightarrow B^{k-1}\right\}_{k \in \mathbb{Z}}$ such that

$$
\varphi_{k}-\psi_{k}=d_{k-1}^{\mathcal{B}} \circ K_{k}+K_{k+1} \circ d_{k}^{\mathcal{A}}
$$

for each $k \in \mathbb{Z}$. Pictorially,

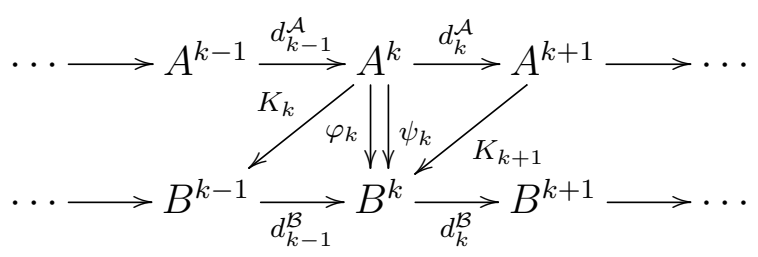

Proposition B.6. Let $\varphi, \psi: \mathcal{A} \rightarrow \mathcal{B}$ be cochain maps, and $K:=\left\{K_{k}\right\}_{k \in \mathbb{Z}} a$ cochain homotopy from $\varphi$ to $\psi$. Then, the induced maps $\varphi_{k}^{\sharp}, \psi_{k}^{\sharp}: H^{k}(\mathcal{A}) \rightarrow H^{k}(\mathcal{B})$ in cohomology are the same, for each $k$.

Proof. For each $k \in \mathbb{Z}$ and for each $a \in \operatorname{Ker}\left(d_{k}^{\mathcal{A}}\right)$,

$$
\varphi_{k}(a)-\psi_{k}(a)=d_{k-1}^{\mathcal{B}}\left(K_{k}(a)\right)+K_{k+1}\left(d_{k}^{\mathcal{A}}(a)\right)=d_{k-1}^{\mathcal{B}}\left(K_{k}(a)\right)
$$

that is, $\varphi_{k}(a)-\psi_{k}(a) \in \operatorname{Im}\left(d_{k-1}^{\mathcal{B}}\right)$. Hence,

$$
\varphi_{k}^{\sharp}([a])=\left[\varphi_{k}(a)\right]=\left[\psi_{k}(a)\right]=\psi_{k}^{\sharp}([a]) .
$$




\section{Bibliography}

[1] A. Berarducci and M. Otero, Intersection theory for o-minimal manifolds. Annals of Pure and Applied Logic 107 (2001), 87-119.

[2] Ricardo Bianconi, Nondefinability results for expansions of the field of real numbers by the exponential function and by the restricted sine function. $J$. Symbolic Logic 62 (1997), no. 4, 1173-1178.

[3] Jan Denef and Lou van den Dries, $p$-adic and real subanalytic sets. Ann. of Math. (2) 128 (1988), 79-138.

[4] Lou van den Dries, Remarks on Tarski's problem concerning $(\mathbb{R},+, \cdot, \exp )$, in Logic colloquium' 82, pp. 97-121, Lolli, Longo, and Marcja, editors, North Holland, 1984.

[5] Lou van den Dries, Tame Topology and o-minimal structures, Cambridge University Press, Cambridge, 1998.

[6] Lou van den Dries and Chris Miller, Geometric categories and o-minimal structures. Duke Math. J. 84 (1996), nº 2, 497-540.

[7] Mário J. Edmundo, O-minimal cohomology and definably compact definable groups, available at https://arxiv.org/abs/math/0012050. Accessed 5 Feb 2018.

[8] Mário J. Edmundo and Nicholas J. Peatfield, o-Minimal Čech cohomology. Q. J. Math. 59 (2008), no. 2, 213-220.

[9] Mário J. Edmundo, Gareth O. Jones, Nicholas J. Peatfield, Sheaf cohomology in o-minimal structures. (English summary) J. Math. Log. 6 (2006), no. 2, $163-179$. 
[10] Mário J. Edmundo, M. Mamino, and L. Prelli, On definably proper maps, Fund. Math. 233 (1) (2016), 1-36.

[11] Andreas Fischer, Smooth functions in o-minimal structures. Advances in Mathematics, 218 (2008), 496-514.

[12] Ziyang Gao, About the mixed André-Oort conjecture: reduction to a lower bound for the pure case. C. R. Math. Acad. Sci. Paris 354 (2016), no. 7, 659-663.

[13] Gareth Jones and Patrick Speissegger. Generating the Pfaffian closure with total Pfaffian functions. J. Log. Anal., 4 (2012), 1-6.

[14] J. Knight, A. Pillay, and C. Steinhorn, Definable sets in ordered structures. II. Trans. AMS 295 (1986), 593-605.

[15] John M. Lee, Introduction to Smooth Manifolds, 2nd Ed., Springer, New York, 2013.

[16] Olivier Le Gal and Jean-Philippe Rolin, An o-minimal structure which does not admit $\mathcal{C}^{\infty}$ celullar decomposition. Ann. Inst. Fourier (Grenoble) 59 (2009), no. 2, 543-562.

[17] Jean-Marie Lion and Patrick Speissegger, Analytic stratification in the Pfaffian closure of an o-minimal structure. Duke Math. J. 103 (2000), no. 2, $215-231$.

[18] Jean-Marie Lion and Jean-Philippe Rolin. Intégration des fonctions sousanalytiques et volumes des sous-ensembles sous-analytiques. Ann. Inst. Fourier 48 (1998), 755-767.

[19] Ib Madsen and Jorgen Tornehave, From Calculus to Cohomology - de Rham cohomology and characteristic classes, Cambridge University Press, New York, 2001.

[20] Ya'acov Peterzil and Charles Steinhorn, Definable compactness and definable subgroups of o-minimal groups. J. London Math. Soc. (2) 59 (1999), no. 3, 769-786.

[21] Jonathan Pila, O-minimality and the André-Oort conjecture for $\mathbb{C}^{n}$. Ann. of Math. (2) 173 (2011), no. 3, 1779-1840. 
[22] Jonathan Pila and Jacob Tsimerman, The André-Oort conjecture for the moduli space of abelian surfaces. Compos. Math. 149 (2013), no. 2, 204-216.

[23] Jonathan Pila and Alex Wilkie, The rational points of a definable set. Duke Math. J. 133 (2006), no. 3, 591-616.

[24] Anand Pillay and Charles Steinhorn, Definable sets in ordered structures I. Trans. AMS 295 (1986), 565-592.

[25] Anand Pillay and Charles Steinhorn, Definable sets in ordered structures III. Trans. AMS 309 (1988), 469-476.

[26] Masahiro Shiota, Abstract Nash manifolds. Proc. Amer. Math. Soc. 96 (1986), no. $1,155-162$.

[27] Patrick Speissegger, The Pfaffian closure of an o-minimal structure. J. Reine Angew. Math. 508 (1999) 189-211.

[28] Alfred Tarski, A Decision Method for Elementary Algebra and Geometry, RAND Corporation, Santa Monica, 1948.

[29] Athipat Thamrongthanyalak, Definable smoothing of continuous functions. Illinois J. Math. 57 (2013), no. 3, 801-815.

[30] Loring W. Tu, An Introduction to Manifolds, 2nd Ed., Springer, New York, 2010 .

[31] Alex J. Wilkie, Covering definable open sets by open cells. O-minimal Structures, Proceedings of the RAAG Summer School Lisbon 2003, Lecture Notes in Real Algebraic and Analytic Geometry (2005).

[32] Alex J. Wilkie, Complex continuations of $\mathbb{R}_{\text {an,exp-definable unary functions }}$ with a diophantine application. J. Lond. Math. Soc. (2) 93 (2016), no. 3, 547-566. 


\section{Index}

$\mathcal{C}^{p}$ global

frame, 51

section, 49

$\mathcal{C}^{p}$ local

frame, 51,53

section, 49, 53

$\mathcal{C}^{p}$-map, 11

$k$-coboundary, 106

$k$-cochain, 106

$k$-cocycle, 106

$k$-covector, 101

$k$-tensor, 101

abstract-definable

$\mathcal{C}^{\infty} k$-forms, 73

$\mathcal{C}^{\infty}$ bump function, 72,74

$\mathcal{C}^{\infty}$ diffeomorphism, 86

$\mathcal{C}^{\infty}$ diffeomorphism invariance, 86

$\mathcal{C}^{\infty}$ homotopy, 90

$\mathcal{C}^{\infty}$ homotopy equivalence, 90

$\mathcal{C}^{\infty}$ manifold, 71

$\mathcal{C}^{p}$ 0-forms, 63

$\mathcal{C}^{p} k$-form, 63

$\mathcal{C}^{p} k$-forms, 64

$\mathcal{C}^{p}$ 1-form, 56

$\mathcal{C}^{p}$ Urysohn's lemma, 33

$\mathcal{C}^{p}$ atlas, 7

$\mathcal{C}^{p}$ bump function, 34

$\mathcal{C}^{p}$ diffeomorphism, 12

$\mathcal{C}^{p}$ manifold, 10
$\mathcal{C}^{p}$ map, 12,34

$\mathcal{C}^{p}$ partition of unity, 30

$\mathcal{C}^{p}$ vector bundle, 48

〜-equivalent atlases, 9

homotopy invariance, 93

map, 12

set, 12,15

alternating

$k$-tensor, 101

anticommutative graded algebra, 103

antiderivation, 73, 103

approximation of definable functions, 29

base space, 48

basis

for the cotangent space, 45

for the exterior product of cotangent spaces, 64

for the tangent space, 40

boolean algebra, 12

Bröcker's problem, 91

chain rule, 42

for abstract-definable maps, 39, 40, 60,61

for definable maps, 38, 39

change of coordinates, 42, 45, 67

closed abstract-definable form, 84

cochain

complex, 105 
homotopy, 108

map, 106

cohomologous abstract-definable forms, 84

cohomology

class, 106

group, 106

of the cochain complex, 106

class, 84

connecting homomorphism, 89, 107

coordinate frame, 51

coordinate frames, 56

cotangent

bundle, 44, 48, 56, 62

space, 44

covector, 44

definable homotopy type, 90

definably

compact, 20

connected, 25

connected components, 84

contractible, 98

homotopic, 90

homotopy equivalent, 90, 97

normal, 19, 31

regular, 19, 21, 34

connected, 23

connected component, 23

normal, 22

definably contractible, 90

differential

of a function, $58,60,66,73,85$

of a map at a point, 39,58

of the cochain complex, 105

dimension of definable sets, 28

directional derivative, 41 dual basis, 45

exact abstract-definable form, 84

exact sequence, 105

exterior algebra, 103

exterior derivative, 73

exterior power of the cotangent bundle, 62

exterior product, 101

fiber, 47

global

exterior derivative, 77

frame, 51

section, 49

graded algebra, 73, 87, 103

Grassmann algebra, 103

homogeneous element, 103

homomorphism of graded algebras, 103

Homotopy Axiom, 93

induced map

in cohomology, 107

induced maps

in cohomology, 93, 108

Jacobian matrix, 42

local

exterior derivative, 75

frame, 51

frame associated with a local trivialization, 52

operator, 74

section, 49

local frame

for the exterior power of the cotangent bundle, 64 
local trivialization, 48

locally constant function, 25

locally definably compact, 20

long exact sequence, 89, 107

manifold topology, 10

Mayer-Vietoris sequence, 89

Monotonicity theorem, 20

o-minimal de Rham

cohomology group, 84

cohomology, 84

partial derivative, 41

permutation, 101

Pfaffian closure, 91

Poincaré lemma, 98

product of abstract-definable manifolds,

11

pullback, 67

map, 73,86

map in cohomology, 86, 87

of an abstract-definable 1-form, 59,

61

of an abstract-definable $k$-form, 69

of an abstract-definable function, 59

of the derivative, 80

of the wedge product, 68

of an abstract-definable 1-form, 60

real exponential field, 98

short exact sequence, 105

of cochain complex, 107

of cochain complexes, 89

straight-line homotopy, 90

tangent

bundle, 43,48 space, 38

vectors, 38

The zig-zag lemma, 107

the zig-zag lemma, 89

total space, 48

trivial vector bundle, 48

trivializing open cover, 48

weak definable $\mathcal{C}^{p}$ version of Urysohn's lemma, 29

wedge product, 65, 101

of abstract-definable forms, 65

of differentials, 66

on the cohomology classes, 86 